Aus der Abteilung Allgemeinmedizin

(Prof. Dr. med. M.M. Kochen, MPH, FRCGP)

im Zentrum Innere Medizin

der Medizinischen Fakultät der Universität Göttingen

\title{
Informationswünsche an ein medizinisches Expertenforum im Internet
}

Quantitative und qualitative Analyse am Beispiel „Kinderwunsch“

\author{
INAUGURAL-DISSERTATION \\ zur Erlangung des Doktorgrades \\ der Medizinischen Fakultät \\ der Georg-August-Universität zu Göttingen \\ vorgelegt von \\ Juliane Meyer \\ aus \\ Quedlinburg
}

Göttingen 2004 
Dekan: Prof. Dr. med. M. Droese

I. Berichterstatter: Priv.-Doz. Dr. disc. pol. W. Himmel

II. Berichterstatter/in: Prof. Dr. med. W. Wuttke III. Berichterstatter/in:

Tag der mündlichen Prüfung: 19. Juli 2004 
1. Einleitung 1

2. Stand der Forschung 2

2.1 Ungewollte Kinderlosigkeit 2

2.1.1 Definition 2

2.1.2 Ursachen 2

2.1.3 Epidemiologie 3

2.1.4 Therapeutische Optionen 3

2.1.5 Risiken der Reproduktionsmedizin 4

2.1.6 Psychosomatische Auswirkungen der Kinderlosigkeit 5

2.1.7 Psychologische Auswirkungen der Reproduktionsmedizin $\quad 7$

2.2 Das Internet als neues Informationsmedium 9

2.2.1 Das Internet als Medium zur Patientenaufklärung und -information 10

2.2.2 Beratung und Aufklärung im Internet durch $\begin{array}{ll}\text { Gesundheitseinrichtungen } & 11\end{array}$

2.3 Bisherige Untersuchungen auf diesem Gebiet 13

3. Fragestellung 15

$\begin{array}{ll}\text { 4. Methoden } & 17\end{array}$

4.1 Retrospektive Auswertung 19

4.1.1 Vorüberlegung 19

$\begin{array}{ll}4.1 .2 \text { Stichprobe } & 19\end{array}$

4.2 Befragung der Nutzer 20

4.2.1 Vorüberlegung 20

4.2.2 Stichprobe 21

$\begin{array}{lll}4.2 .3 & \text { Fragebogen } & 22\end{array}$

4.2.3.1 Entwicklung 22

4.2.3.2 Aufbau 22

4.2.3.3 Vorstellen der Studie 23

4.2.3.4 Selektion der Studienteilnehmer 24

4.2.3.5 Fragen zum Besuch des Expertenforums 25

4.2.3.6 Derzeitige Versorgungssituation 25

4.2.3.7 Nutzerprofil 25

4.2.3.8 Technische Präsentation 26

4.3 Pilotphase 26

4.4 Durchführung der Untersuchung 27

$\begin{array}{ll}4.5 \text { Datenschutz } & 27\end{array}$ 
4.6 Auswertung 28

4.6.1 Quantitative Auswertung 29

$\begin{array}{ll}\text { 4.6.2 Qualitative Auswertung } & 29\end{array}$

4.6.3 Verknüpfung von selektiven Einzelfallanalysen und quantitativem Datenmaterial

5.1 Auswertung der Pilotphase 33

5.2 Internet-Befragung 33

5.2.1 Ausschöpfung der Stichprobe 33

5.2.3 Kontakt mit dem Expertenforum 36

5.2.4 Gründe für den Besuch des Expertenforums 37

5.2.5 Bewertung des Expertenforums 37

5.2.6 Expertenforum und Arztbesuch 38

5.2.7 Dauer und Behandlung der Kinderlosigkeit 38

5.2.8 Bewertung der ärztlichen Betreuung und Information 39

5.3 Inhaltsanalytische Auswertung der Anfragen 42

5.3.1 Stichprobenumfang 42

5.3.2 Funktion des Expertenforums 42

5.3.2.1 Aufklärung 45

5.3.2.2 Unabhängige medizinische Beratung 48

5.3.2.3 Lotsenfunktion 51

5.3.2.4 Emotionale Unterstützung 52

5.3.3 Stichprobe der Männer 54

5.3.4 Implizite Bewertung des Expertenforums 55

5.3.5 Bewertung der ärztlichen Betreuung 55

5.3.6 Einzelfallanalysen 56

5.3.6.1 Beispiel einer beginnenden Hilfesuche 57

5.3.6.2 Beispiel eines langen Therapieweges 57

5.3.6.3 Beispiel einer erfolgreichen Hilfesuche 59

6. Diskussion $\quad 61$

6.1 Diskussion der Methode 61

6.1.1 Die methodische Triangulation 61

6.1.2 Gütekriterien 62

6.1.3 Quantitative Erhebung: Online-Befragung 63 
6.1.4 Inhaltsanalytische Auswertung 68

6.2 Diskussion der Ergebnisse 69

$\begin{array}{ll}6.2 .1 & \text { Stichprobe } \\ \end{array}$

6.2.2 Signalisierter Hilfebedarf 70

$\begin{array}{ll}\text { 6.2.3 Nutzung des Expertenforums } & 72\end{array}$

6.2.4 Bewertung des Internetangebots $\quad 74$

$\begin{array}{ll}6.3 \text { Ausblick } & 77\end{array}$

7. Zusammenfassung $\quad 80$

8. Anhang $\quad 82$

9. Literaturverzeichnis $\quad 95$ 


\section{Einleitung}

Das Internet als Quelle für medizinische Informationen wird immer populärer, je mehr medizinische Laien die Möglichkeit haben, „online zu gehen“. Dem Internet-Nutzer steht eine wachsende Zahl an so genannten „health pages“ mit Gesundheitsinformationen und Dienstleistungen zur Verfügung. Das Internet gewinnt damit als integraler Bestandteil der medizinischen Versorgung an Bedeutung.

Viele Paare mit Kinderwunsch stürzen in eine schwere Lebenskrise, wenn sie bemerken, dass sich die ersehnte Schwangerschaft nicht einstellt. Das kann zur Folge haben, dass - manchmal verzweifelt - Informationen zum Thema „Kinderwunsch“ gesammelt werden. Kinderlose Paare sind mit zahlreichen Websites von Gesundheitsorganisationen, Fachgesellschaften, Reproduktionskliniken, pharmazeutischen Herstellern und Selbsthilfegruppen konfrontiert. Wie umfangreich die Datenmenge im Internet ist, verdeutlicht die Eingabe des Wortes „Kinderwunsch“ in die Suchmaschine Google („www.google.com“): Der Online-User erhält weltweit 233.000 Treffer (zugegriffen am 08/02/2004).

Eine vergleichsweise neue Variante im Internet sind Online-Beratungen durch qualifizierte Ärzte. Über die Gründe der Nutzung dieser so genannten Expertenforen und ihre Bewertung durch die Nutzer ist wenig bekannt. Ziel dieser Arbeit ist es, einen Überblick über den Wissensbedarf von Besuchern eines Expertenforums zum Thema Kinderwunsch und daraus indirekt einen Überblick über die derzeitige Betreuungssituation ungewollt kinderloser Paare zu erhalten. Aus den Nutzungserfahrungen der Besucher sollte darüber hinaus das Internetangebot hinsichtlich seiner Funktion und möglichen Rolle (stellvertretend) für die zukünftige Medizin eingeschätzt werden. 


\section{Stand der Forschung}

\section{$2.1 \quad$ Ungewollte Kinderlosigkeit}

\subsubsection{Definition}

Von einer Sterilität wird gesprochen, wenn innerhalb eines Zeitraums von einem Jahr bei regelmäßigem ungeschützten Geschlechtsverkehr keine Schwangerschaft eingetreten ist. Infertilität ist definiert als Unfähigkeit eine Schwangerschaft auszutragen (Fischl 2000). Häufig werden diese Begriffe in der Literatur auch synonym gebraucht.

\subsubsection{Ursachen}

Die wesentlichen Ursachen ungewollter Kinderlosigkeit sind zwischen Mann und Frau annähernd gleich verteilt. In $20 \%$ der Fälle treten Störungen bei beiden Partnern auf. Auf Seiten der Frau werden im Wesentlichen zwei Sterilitätsursachen unterschieden:

- Störungen der Ovarialfunktion: diese sind durch das Ovar selbst oder durch Störungen in der hypothalamisch-hypophysären Achse bedingt und werden als Amenorrhoe oder Menstruationsstörung manifest.

- Störungen der Tuben: diese werden häufig durch entzündliche Erkrankungen der Tuben und den dadurch bedingten partiellen oder kompletten Tubenverschluss hervorgerufen. Andere Ursachen sind Dyskinesie der Tuben durch endokrine Störungen, Endometriose der Eileiter oder Tumore.

Seitens des Mannes führen folgende Ursachen häufig zu einer Zeugungsunfähigkeit:

- $\quad$ endokrine Störungen, wie Störungen in den hypothalamisch-hypophysären Regulationszentren

- $\quad$ testikuläre Ursachen; dazu gehören u.a. die angeborene oder erworbene Anorchie, der Hodenhochstand, Varikozele und Orchitis (Infektion mit Zerstörung des Keimepithels), aber auch Steroidsynthesestörungen in den Leydig-Zellen und 
Funktionsstörungen der Sertolizellen sowie resultierende Störungen in der Spermatogenese.

Als Ursache ungewollter Kinderlosigkeit sind oft zahlreiche Faktoren, die sich wechselseitig bedingen, wirksam. Neben den erwähnten spezifischen organischen Störungen (Hinney und Michelmann 2001; Nieschlag und Behre 1996) kommen dabei auch unspezifische, wie z. B. psychologische oder soziale Aspekte zum Tragen (Schill und Haidl 1995; Bengel et al. 2000; Henning und Strauß 2002).

\subsubsection{Epidemiologie}

Etwa 10-15\% aller Paare im reproduktionsfähigen Alter gelten - zumindest vorübergehend - als ungewollt kinderlos (Strauß et al. 1991; Hinney und Michelmann 2001). Um diese vergleichsweise hohe Zahl angemessen einzuschätzen und zu bewerten, sollte man Folgendes berücksichtigen:

Regelmäßiger, ungeschützter Geschlechtsverkehr vorausgesetzt, liegt die Schwangerschaftsrate bei ungefähr 25\% pro Zyklus (Tinneberg 1995). Dieser Wert schwankt in Abhängigkeit vom Alter der Frau, von der Lebensweise (Nikotin- und Alkoholmissbrauch), dem Stress, der psychischen Verfassung und von schwer objektivierbaren Faktoren wie „Umwelteinflüssen" (Heinzow und Hanf 1995; Fischl 2000). 60-70\% der Paare mit Kinderwunsch können in der Regel innerhalb eines Jahres ihren Wunsch realisieren (Fischl 2000). Am Ende ihrer reproduktiven Phase bleiben rund 3-4\% aller Kinderwunschpaare ungewollt kinderlos (Templeton 1992). Brähler und StöbelRichter (2002) haben diesen Wert bestätigt und davor gewarnt, die Rate ungewollter Kinderlosigkeit - durch Verwechslung vorübergehender und dauerhafter Kinderlosigkeit - zu dramatisieren.

\subsubsection{Therapeutische Optionen}

Bei Störungen der Ovarialfunktion werden verschiedene hormonelle Stimulationsverfahren eingesetzt. Begonnen wird in der Regel mit einer Antiöstrogentherapie (z. B. mit Clomifen), welche die hypophysäre Gonadotropinausschüttung stimuliert und so die Ovarialfunktion fördert. Ist die Behandlung mit Clomifen erfolglos, wird die Therapie mit Gonadotropinen fortgesetzt (Hinney und Michelmann 2001). 
Bei verschlossenen oder anderweitig beeinträchtigten Tuben kommen die mikrochirurgische Rekonstruktion der Tuben oder die In-vitro-Fertilisation (IVF) ${ }^{1}$ in Betracht. Die Lebendgeburtsrate („,baby-take-home“-Rate) beträgt 16,9\% pro Behandlungszyklus (Human Fertilisation and Embryology Authority, HFEA 2000). Voraussetzung für eine erfolgreiche IVF ist eine ausreichende Samenqualität des Ehemannes bzw. Partners.

Werden bestimmte Mindestanforderungen der Spermienqualität unterschritten, steht die intrazytoplasmatische Spermatozoeninjektion $(\mathrm{ICSI})^{2}$ zur Verfügung. Seit Etablierung von ICSI ist die einzige Anforderung an die Ejakulatqualität die Präsenz eines einzigen lebenden Spermatozoons pro Eizelle. ICSI kann bei Azoospermie erfolgreich eingesetzt werden (Tinneberg 1995; Hinney und Michelmann 2001). Zudem steht selbst Patienten mit fehlenden Spermatozoen im Ejakulat und nachweisbaren testikulären Schaden durch moderne Methoden der assistierenden Reproduktion die Erfüllung ihres Kinderwunsches offen. Die „baby-take-home“-Rate liegt etwa gleichauf mit der IVF (Hinney und Michelmann 2001). Durch Kryokonservierung von Spermatozoen aus Hoden und Nebenhoden für die spätere ICSI ist die Verfügbarkeit der männlichen Keimzellen nicht mehr an den Zeitpunkt ihrer Entnahme gekoppelt.

Die Entscheidung, welche Therapie angewendet wird, sollte unter Berücksichtigung der individuellen Situation nach Absprache mit dem betroffenen Paar gefällt werden.

\subsubsection{Risiken der Reproduktionsmedizin}

Seit der Einführung der ICSI gab es immer wieder Bedenken, dass durch die Spermieninjektion in die Eizelle das Risiko einer Missbildung des Kindes steigt: Neuere amerikanische Untersuchungen ergaben eine doppelt so hohe Fehlbildungsrate bei durch ICSI gezeugten Kindern (Hansen et al. 2002). So traten bei 9\% aller durch ICSI gezeugten Kinder Fehlbildungen wie z. B. chromosomale, muskuloskeletale sowie kardiovaskuläre Defekte auf. Im Vergleich dazu kam es unter den normal gezeugten Kinder bei 4,2\% zu Fehlbildungen (Hansen et al. 2002). Deutsche Studien hingegen zeigten ein 1,3-fach erhöhtes Fehlbildungsrisiko bei „ICSI-Kindern“ im Ver-

\footnotetext{
1,2 Zur Beschreibung der Technik z. B. Tinneberg (1995)
} 
gleich zu einem Normalkollektiv (Ludwig 2002). Studien zur IVF kamen zu ähnlichen Ergebnissen (Hansen et al. 2002). Nicht evident ist, ob das bis zu doppelt so hohe Fehlbildungsrisiko bei der ICSI-Behandlung durch das Verfahren selbst oder auch bzw. allein durch die Risikofaktoren - insbesondere das höhere Alter - der zukünftigen Eltern einer ICSI-Therapie verursacht wird (Ludwig 2002). Eine klare Korrelation besteht zwischen IVF/ICSI und Frühgeburten. Zudem haben amerikanische Wissenschaftler (Schieve et al. 2002) ermittelt, dass in vitro gezeugte Kinder mit einem geringeren Geburtsgewicht zur Welt kommen. Ob die bei künstlicher Befruchtung häufig vorkommenden Mehrlingsschwangerschaften und Frühgeburten die einzigen Gründe für ein signifikant niedriges Geburtsgewicht unter ICSI oder IVF sind, ist noch nicht geklärt.

\subsubsection{Psychosomatische Auswirkungen der Kinderlosigkeit}

Ein unerfüllter Kinderwunsch stellt für viele Paare eine schwere Lebenskrise dar, da Kinderlosigkeit häufig als Makel, Schande und tiefe Kränkung empfunden wird (Menning 1980). Auch wenn psychische und soziale Reaktionen eines Paares mit der Diagnose Infertilität bzw. Sterilität sehr unterschiedlich sein können, bewältigen betroffene Paare ihre Trauer - wie stresstheoretische Ansätze und Copingmodelle zeigen in aufeinander folgenden Stadien (Menning 1980; Hölzle 1988; Callan und Hennessey 1989):

- Schock durch die ärztliche Diagnose Unfruchtfruchtbarkeit: Die Paare stellen fest, dass sie nun die Möglichkeit, ein Kind zu zeugen, verloren haben und möglicherweise für immer in ihrem Leben auf leibliche Kinder verzichten müssen. Gefühle wie Verzweiflung und Hoffnungslosigkeit sind bestimmend.

- Verneinung: In dieser Phase werden immer neue medizinische Untersuchungen begonnen, um die bestehende Diagnose zu revidieren.

- Ärger, Wut und Frustration über die Erfahrungen beim Arztbesuch und bei medizinischen Behandlungen sind in der dritten Phase bestimmend. Die eigene vermeintliche Unzulänglichkeit kann gegenüber dem sozialen Umfeld irrationale Reaktionen hervorrufen, wie z. B. Aggressionen gegen Paare mit Kindern. 
- Isolation: gekennzeichnet vom Abbruch aller sozialen Kontakte vor allem zu Bekannten mit Kindern.

- Schuld- und Schamgefühle: Diese Phase ist geprägt durch die Suche nach der Ursache der Unfruchtbarkeit. Vor allem in der Vergangenheit wird nach einem Auslöser des Defizits (Kontrazeptiva-Gebrauch, Abtreibung) geforscht.

- Depressionen und anschließende Trauergefühle, durch die ein Verarbeitungsprozess in Gang gesetzt wird.

Die Frage, ob Männer und Frauen gleich stark durch die Kinderlosigkeit belastet werden, wurde bis jetzt nicht eindeutig beantwortet (Küchenhoff 1999). Wright et al. (1991) verneinen eine gleichstarke Belastung von Männern und Frauen und sehen die ungewollte Kinderlosigkeit für Frauen als belastender an. Bernt et al. (1992) und Kowalcek et al. (2000) identifizierten geschlechterspezifische Copingstrategien zur Bewältigung von Fertilitätsstörungen und Kinderlosigkeit: Frauen suchen in höherem Maße als Männer nach Informationen, z. B. lesen sie in Fachbüchern oder Ratgebern (Berg BJ et al. 1991). Frauen versuchen häufiger, medizinisches Wissen in Bezug auf ihre Sterilität zu erwerben (Boeger et al. 1999). Männer geben sich kontrollierter und distanzierter, während Frauen sich stärker um soziale Unterstützung von Verwandten und Freunden bemühen und häufiger Kontakt zu anderen Betroffenen pflegen (Abbey et al. 1991; Berg BJ et al. 1991; Stanton et al. 1992; Schröter et al. 1998). Im Gegensatz dazu meiden sie häufiger als Männer Menschen und Orte, die sie mit ihrer Kinderlosigkeit konfrontieren könnten (Greil et al. 1988; Berg BJ et al. 1991; Stanton et al. 1991). Depressive Verstimmungen, psychosomatische Auffälligkeiten und geringeres Selbstwertgefühl sind bei Frauen stärker ausgeprägt, während Männer die mit dem Kinderwunsch einhergehenden Probleme tendenziell verleugnen.

Frauen übernehmen signifikant häufiger als Männer die Verantwortung für die ungewollte Kinderlosigkeit, unabhängig von den tatsächlichen medizinischen Ursachen der Infertilität (McEwan et al. 1987; Berg BJ et al. 1991; Abbey und Halman 1995; Bernt et al. 1992). Sie betrachten z. B. die ungewollte Kinderlosigkeit als Strafe für ihre in der Vergangenheit begangenen "Fehler", wie Schwangerschaftsabbrüche oder Empfängnisverhütung (Berg BJ et al. 1991). Greil et al. (1988) erklären dies im 
Sinne eines erlebten Rollenversagens, das alle Lebensbereiche beeinflusst. Daher würden Frauen auch häufiger die Initiative bei der Suche nach medizinischer Hilfe ergreifen (Berg BJ et al. 1991).

Männer messen insgesamt persönlichen Ursachen weniger Bedeutung zu als Frauen (Greil et al. 1988, Bernt et al. 1992, Kowalcek et al. 2000). Das Belastungserleben des Mannes korrespondiert mit der psychischen Belastung seiner Partnerin bzw. mit Unstimmigkeiten in der Partnerschaft (Greil et al. 1988, Stanton et al. 1991). Unabhängig davon, bei welchem Partner die Ursachen der Kinderlosigkeit - medizinisch gesehen - liegen, schreiben Männer die Verantwortung für die Kinderlosigkeit verstärkt ihren Frauen zu (Abbey und Halman 1995).

Die Belastung durch ungewollte Kinderlosigkeit erleben Frauen und Männer verstärkt, wenn die Diagnose ungenau ist, bei Partnerschaftsproblemen (Nijs et al. 1984), bei bestimmter Religionszugehörigkeit (Pennanen 1993), wenn die betroffenen jünger (McEwan et al. 1987) oder sozial isoliert sind (Nijs et al. 1984) und wenn sie sich in medizinischer Behandlung befinden (McEwan et al. 1987).

\subsubsection{Psychologische Auswirkungen der Reproduktionsmedizin}

Die hochtechnisierte reproduktionsmedizinische Behandlung kann für ungewollt kinderlose Paare auf mehreren Ebenen zu einem sehr belastenden Lebensereignis werden:

1. Auf der individuellen Ebene müssen Männer oder Frauen eher mit einem Misserfolg als mit einem Erfolg der Behandlung rechnen: Der Therapieerfolg bei IVF liegt laut HFEA (2000) pro Zyklus unter 20\%; die kumulierte, also durch mehrfache Versuche erzielte Erfolgsrate liegt bei über 50\%. Darüber hinaus sind für beide Partner individuell direkte physische und psychische Auswirkungen der Behandlung zu verarbeiten (Leiblum et al. 1987; Strauß et al. 1991).

2. Auf der Paarebene kann die Partnerschaft in Mitleidenschaft gezogen werden, wenn z. B. das Leben des Paares fast nur noch auf die Erfüllung des Kinderwunsches ausgerichtet ist (Kentenich et al. 1987; Andrews et al. 1991; Pennanen 1993). 
3. Auf der sozialen Ebene muss sich das Paar mit den oftmals ablehnenden oder wenig hilfreichen Reaktionen aus seinem Umfeld (z. B. Verwandte, Freunde, Arbeitskollegen) auseinandersetzen (Kentenich et al. 1987; Schröter et al. 1998; Onnen-Isemann 2000).

In zahlreichen Studien wurde das Warten in der behandlungsfreien Zeit bis zum Schwangerschaftstest oder zur Fertilisation als besonders belastend beschrieben (z.B. Freeman et al. 1987; Strauß et al. 1991). Weitere objektive Belastungen während der reproduktionsmedizinischen Behandlung sind der terminliche und organisatorische Aufwand für Paare, um z. B. die eigenen Arbeitszeiten dem Ablauf in der Arzt- oder Kinderwunsch-Klinik anzupassen (Henning und Strauß 2002).

Im Vordergrund der psychischen Beeinträchtigung stehen depressive Reaktionen, Nervosität, Stress und Angst vor Misserfolg (Kentenich et al. 1987; Strauß et al. 1991). Sexuelle Probleme und Unstimmigkeiten in der Partnerschaft wurden ebenfalls als erhebliche Stressfaktoren angegeben (Strauß et al. 1991; Henning und Strauß 2002). Psychische Probleme sind bei allen Verfahren prinzipiell abhängig von der Erfolgsrate zu sehen, aber auch davon, in welchem Verhältnis der Erfolg zum Aufwand der beteiligten Person steht. So werden invasive Eingriffe wie ICSI und IVF als wesentlich belastender erlebt als weniger invasive Methoden wie Insemination oder Ovulationsstimulation durch Clomifen (Schuhrke 1993).

Zu den speziellen Reaktionsweisen und Belastungen von Männern in einer Fertilitätstherapie gibt es kaum empirische Untersuchungen. Generell scheinen die Männer ihr psychisches Erleben mehr in Bezug zu ihrer Frau zu setzen (Felder et al. 2000). Als spezifische Reaktionen bei Männern in der IVF-Therapie gelten z. B. Schuldgefühle gegenüber der Partnerin, Gefühle des Ausgeschlossenseins und Ängste vor Potenzverlust (Stauber et al. 1986). Auch die Spermagewinnung/-abgabe kann emotional sehr belastend sein (Lalos et al. 1986).

Die erwähnten Studien konzentrieren sich auf körperliche und psychische Beeinträchtigungen in zeitlicher Nähe zum aktuellen Kinderwunsch: Belastungen und Einschränkungen im gesundheitlichen und psychischen Wohlbefinden, in der Partnerschaftszufriedenheit, im sozialen Umfeld und in der Lebenszufriedenheit (Kentenich 
et al. 1987; Strauß et al. 1991). Demgegenüber beschäftigen sich nur wenige Studien mit den langfristigen gesundheitlichen Folgen von ungewollter Kinderlosigkeit und den psychischen Beeinträchtigungen infertiler Paare im späten Erwachsenenalter. Im sozialen Bereich fehlt den kinderlosen Männern und Frauen emotionale Unterstützung; im späten Erwachsenenalter bzw. im hohen Alter und in Krisensituationen (z. B. bei schwerer Krankheit) sind sie stärker als Eltern von sozialer Isolation und Einsamkeit bedroht (Johnson und Catalano 1981; im Überblick Strauß et al. 2000). Insgesamt bestehen nach heutigem Kenntnisstand nur geringe Unterschiede in der Lebenssituation (Gesundheit, psychisches Wohlbefinden, Lebenszufriedenheit) von Eltern und Kinderlosen im späten Erwachsenenalter und Alter (Keith 1983; Boeger et al. 1999; Bengel et al. 2000; Strauß et al. 2000).

\subsection{Das Internet als neues Informationsmedium}

Das Internet hat sich im Laufe der letzten Jahre zu einer vielgenutzten Informationsund Kommunikationsplattform entwickelt. Es dient mittlerweile dem weltweiten Informationsaustausch in Forschung und Wirtschaft sowie zwischen Privatpersonen (Theobald 2000).

Der Zuwachs der Internetnutzung ist vor allem in Deutschland und Europa weiterhin enorm. Laut Nielsen/NetRatings (2002 a) umfasste die „Gemeinde“ der Internetnutzer im März 2002 weltweit fast 500 Millionen Menschen. Im Jahr 2005 soll die Zahl der an das Internet angebundenen Rechner die Milliardengrenze erreichen (Electronic Commerce Info Net, ECIN 2002). In Deutschland wurden im Mai 2002 knapp 27 Millionen Internetnutzer über 14 Jahren registriert (Emnid 2002). Der jährliche Zuwachs beträgt rund 4,5\%. Der Anteil der Frauen unter den Internetnutzern in Deutschland lag im Januar 2002 bei rund 39\% (Nielsen/NetRatings 2002 b)

Unterrepräsentiert sind Bevölkerungsgruppen mit formal geringerem Bildungsniveau und niedrigerem Einkommen (Nielsen/NetRatings 2002 a; European Opinion Research Group, EORG 2003). Im Jahr 1995 waren 95\% der Internetnutzer Abiturienten. Diese Zahl ist bis Frühjahr 2000 auf 62,3\% gesunken. Daraus ist zu schließen, dass zunehmend Menschen aus allen sozioökonomischen Schichten im Internet „surfen“ und die „sozial übergreifende“ Nutzung des Internets steigt (Weissmann et al. 2000; EORG 2003). 
Die sich ausbreitende digitale Vernetzung von Millionen Menschen weltweit legt nahe, das Internet, insbesondere das World Wide Web, auch in der Primärforschung zur aktiven Generierung bisher noch nicht erhobener Daten zu nutzen (Batinic und Bosnjak 1997; Werner 1997; Theobald und Brabänder 1998).

\subsubsection{Das Internet als Medium zur Patientenaufklärung und -information}

Zurzeit stehen dem Online-User und (potentiellen) Patienten über 3 Millionen Webseiten zur Verfügung (Google 2003; zugegriffen am 01/09/2003), davon enthalten 2\% gesundheitsrelevante Themen (Kiley 1999). Für den Verbraucher reicht das Angebot an Webseiten mit Schwerpunkt Gesundheit von kommerziellen Gesundheitsportalen über E-Commerce-Seiten, Gesundheits-Communities, Online-Journals, Seiten von Fachverbänden, Selbsthilfegruppen oder Patientenverbänden, Forschungseinrichtungen und Universitäten, Suchmaschinen für gesundheitsrelevante Themen, OnlineApotheken bis hin zur Online-Beratung durch qualifizierte Fachärzte (Weissmann et al. 2000; Köhler und Eysenbach 2002).

Der Inhalt vieler so genannter "health pages" ist zum großen Teil nicht wissenschaftlich fundiert, irreführend und/oder veraltet (Silberg et al. 1997, Theobald 2000). Diese Aussage gilt nicht nur für die Medizin, sondern für alle Themenbereiche im World Wide Web. Eysenbach und Diepgen (1999 a) sehen als Ursache für die mangelnde Qualität der Online-Informationen, dass jeder im Internet ohne redaktionelle Kontrolle publizieren kann. Oftmals verstecken sich hinter Webseiten dubiose Anbieter von Gesundheitsprodukten und Arzneimitteln oder Pharmawerbungen („Infomercials“ und „Advertorials“; Eysenbach und Diepgen1999 a).

Für Deutschland gibt es kaum repräsentative Studien über die Nutzung des Internets bei Gesundheitsproblemen. Die folgenden Aussagen beziehen sich vor allem auf die USA und Großbritannien.

Im Rahmen einer Studie der European Opinion Research Group (EORG 2003) wurden Einwohner der europäischen Union nach ihren bevorzugten Informationsquellen für Gesundheitsthemen gefragt. Als wichtigste Quellen wurden die Fachleute des Gesundheitssystems (Ärzte, Apotheker), das Fernsehen und Fachliteratur angegeben. Das Internet wurde von relativ wenigen Befragten (23,1\%) als Informationsquel- 
len genutzt. Trotzdem stand ein großer Teil der europäischen Bürger (41,5\%) der Online-Suche nach Informationen über Gesundheitsprobleme positiv gegenüber (EORG 2003).

Schätzungen über die Anzahl der Konsumenten in den USA, die das medizinische Angebot im WWW nutzen, reichen von $50-75 \%$ aller Personen mit Internetzugang (Murero et al. 2001; Taylor H 2001; Köhler und Eysenbach 2002). Auf der Suche nach Gesundheitsinformationen „surfen“ Frauen deutlich häufiger im Internet als Männer (Taylor H 2001).

Gerade im Gesundheitsbereich zielt die Suche weniger auf allgemeine Auskünfte im Netz, sondern auf spezifische Informationen zu einer bestimmten Krankheit. Die User haben eine sehr konkrete Vorstellung davon, welche Informationen sie wünschen. Eine Studie von Scherzer et al. (2001) ergab, dass rund 52\% der befragten Internetnutzer nach aktuellen Forschungsergebnissen, 42\% nach Expertenrat und 40\% nach Hintergrundinformationen zu bestimmten Krankheiten suchen.

Für die nächsten Jahre vermuten die Autoren einen weiteren Aufwärtstrend auf dem Gebiet der Patienteninformation über das Internet. Für das Nutzungsverhalten in Deutschland werden ähnliche Tendenzen erwartet (NUA internet surveys 2000).

\subsubsection{Beratung und Aufklärung im Internet durch Gesundheitseinrichtungen}

Das Angebot von Informationen kann eindeutig kommerziell motiviert sein (z. B. die Gesundheitsportale "Netdoktor" (www.netdoktor.de) oder "Meine Gesundheit" (www.meine-gesundheit.de); es kann aber auch von Universitäten, medizinischen Fachgesellschaften, Krankenhäusern und Krankenkassen stammen - mit einem expliziten Öffentlichkeitsauftrag, ggf. mit indirektem kommerziellen Hintergrund. Die Arbeitsgemeinschaft der Wissenschaftlichen Medizinischen Fachgesellschaften (AWMF [www.awmf-online.de]) hat z. B. zu zahlreichen Krankheiten Leitlinien - auch für Patienten - veröffentlicht. Diese Leitlinien informieren Patienten über Einzelheiten ihrer Erkrankung und die verschiedenen Therapiemöglichkeiten.

Ziel der Patienteninformation im Internet ist es, Betroffenen und Angehörigen bei der Entscheidungsfindung für oder gegen die Inanspruchnahme von Diagnoseverfahren 
und Therapiemaßnahmen zu unterstützen, indem aktuelle wissenschaftliche Erkenntnisse für den medizinischen Laien verständlich zur Verfügung gestellt werden.

Je nach eingesetztem Internet-Dienst wurden verschiedene Beratungsservices eingerichtet. Anfragen von Internetnutzern können per E-Mail beantwortet und die Fragen und Antworten anonym im WWW veröffentlicht werden. Der SexualAufklärungsdienst von Pro Familia ist z. B. eine E-Mail-Beratung, die nach diesem Prinzip arbeitet (www.profa.de/f_sextra.htm).

Eine andere Möglichkeit ist die direkte Kommunikation zwischen Experten und Online-User (oder zwischen den Usern selbst) in themenspezifischen Chatrooms oder Foren. Chronisch Kranke und Behinderte haben besonders großen Informations- und Kommunikationsbedarf, dem in zahlreichen Selbsthilfegruppen entsprochen wird (Eysenbach 1999 a). Diese Selbsthilfegruppen haben sich bereits Internet-Foren geschaffen (z. B. die Rheuma-Liga [www.rheuma-liga.de] oder die Migräneliga [www.migraeneliga-deutschland.de]), in denen Betroffene online Informationen einholen und sich mit anderen Betroffenen beraten können.

Durch die Vielzahl an kommerziellen Anbietern, staatlichen Behörden, an Instituten und Krankenkassen - alle mit unterschiedlichen Interessen - kommt es zu Qualitätseinbußen (Eysenbach und Diepgen 1999 a; Kacher et al. 2000; Köhler und Eysenbach2002). Aus diesem Grund fördert die Europäische Kommission die Initiative MedCERTAIN (MedPICS Certification and Rating of Trustworthy and Assessed Health Information on the Net; www.medpics.org/med certain/), die ein Qualitätssiegel für Gesundheitsinternetportale (www.medcertain.org) entwickelt (Kacher et al. 2000). Eine ähnliche Initiative liegt der Arbeitsgruppe DISCERN (www.discern.com; www.discern.de; Charnock 2000) zugrunde: Anbietern von Fachinformationen ein Instrument zur Überprüfung der Qualität ihrer Informationen zur Verfügung zu stellen (s.a. www.patienten-information.de).

Problematisch ist die Beratung durch so genannte "Cyberdocs": Zum einen ist es deutschen Ärzten laut Gesetzgebung nicht erlaubt, per E-Mail oder anderen OnlineKontakten Diagnosen zu erstellen und Medikamente zu verschreiben (BÄK, Musterberufsordnung für Ärzte [MBO], §7, Par. 3, Bundesärztekammer 1997). Zum anderen 
handelt es sich bei den „Cyberdocs" einiger Foren bisweilen um kommerzielle Händler ominöser Gesundheitsprodukte (Eysenbach und Diepgen 1999 a).

\subsection{Bisherige Untersuchungen auf diesem Gebiet}

Studien zur Nutzung des Internets durch Patienten liegen vor allem aus dem Ausland vor. Die Mehrzahl dieser Studien wurde auf traditionelle Weise als Telefonumfrage oder schriftlich mit einem Fragebogen durchgeführt: Taylor MRG et al. (2001) entwarfen einen Fragebogen zur Inanspruchnahme des Internets durch Patienten, die sich in Kliniken, die auf genetische Erkrankungen spezialisiert sind, behandeln ließen. Ein ähnliches Design hatten Studien von Weissman et al. (2000), die die Nutzung des Internets durch kinderlose Paare untersuchten oder von O'Connor und Johanson (2000) zur Internetnutzung von gastroenterologischen Patienten. Eine Telefonumfrage zur Inanspruchnahme des Internet als Quelle medizinischer Informationen wurde z. B. von Murero et al. (2001) bei Patienten nach Herzoperationen durchgeführt. Resultat dieser Studien war, dass die Hälfte der befragten Personen - davon hauptsächlich Frauen - das Internet zur medizinischen Informationssuche nutzten. Im Internet wurde vor allem nach verständlichen Informationen über die jeweiligen Erkrankungen, Behandlungsstrategien und neuesten Forschungsergebnissen gesucht. In allen Studien standen die Befragten der Kommunikation mit ihrem Arzt über das Internet positiv gegenüber und begrüßten einen Ausbau dieser Art von „Arzt-PatientKommunikation“.

Andere Arbeiten beschäftigten sich mit speziellen (nicht-)kommerziellen Internetangeboten. In diesen Studien wurde hauptsächlich der Nutzen multimedialer Vernetzung verschiedener Spezialisten in Form von Diskussions- oder Expertenforen für Ärzte untersucht. Kedar et al. (2003) analysierten die Inhalte von Anfragen an ein Internet-basierendes Forum, welches sich aus verschiedenen Ärzten und Spezialisten zusammensetzte. Die Anfragen stammten von Ärzten und bezogen sich auf ihre Patienten. Dabei ging es vorwiegend um onkologische Sachverhalte und neue Therapiemöglichkeiten, vor allem neue Strategien in der Chemotherapie. Ähnliche Studien wurden von Kronz et al. (1999) und Brauchli et al. (2002) durchgeführt. Diese Studien beschäftigen sich mit der Bedeutung von Tele-Pathologiesystemen im Internet für den behandelnden Arzt und indirekt für den Patienten. 
Wenige Untersuchungen beschäftigen sich mit Internetangeboten, die sich speziell an den Bedürfnissen von medizinischen Laien orientieren. Epstein et al. (2002) verglichen in einem auf einer Website der Infertilitätsorganisation INCIID integrierten Fragebogen die demographischen Daten, den medizinischen Status, Internetnutzung und das psychische Wohlbefinden von kinderlosen Paaren, die nur über das Internet in Selbsthilfeforen kommunizieren und Paaren, die verschiedene Ansprechpartner hatten. Die Untersuchung war zwar in eine patientenorientierte Website integriert, der Fragebogen selbst bezog sich aber nicht auf die Nutzung der Website des INCIID, sondern auf das Online-Verhalten im Ganzen. Spezielle Bedürfnisse medizinischer Laien nach verständlichen Informationen untersuchten Widman und Tong (1997). Sie analysierten die Themeninhalte von 70 E-Mails an Kardiologen einer Website mit dem Schwerpunkt Herzrhythmusstörungen. In der überwiegenden Zahl der E-MailAnfragen wurde um eine zweite Meinung zu Behandlungsmethoden, Diagnosen und Prognosen gebeten. Die Qualität dieser Seite wurde nicht bewertet.

Die oben beschriebenen Studien beziehen sich fast ausnahmslos auf die Nutzung des Internets im Allgemeinen. Wie Medline-Recherchen ergaben, fehlt es an Untersuchungen, die - mit Blick auf ein bestimmtes Internetangebot bzw. Expertenforum die Gründe der Inanspruchnahme und gleichzeitig die Bewertung dieses Angebots durch die Nutzer erheben. 


\section{Fragestellung}

Das Thema „Kinderwunsch“ ist für eine Internetberatung prädestiniert: (1) Kinderlosigkeit ist ein sehr belastendes und verunsicherndes Lebensereignis - für die Betroffenen, die Partnerschaft und das familiär-soziale Umfeld; (2) es gibt verschiedene, teils konträre Ansichten über die Behandlung der Kinderlosigkeit und hinsichtlich ihrer Erfolgschancen; (3) je nach Art bzw. Stadium von Diagnose und Behandlung können verschiedene medizinische und/oder psychologische Berufsgruppen in die Betreuung involviert sein $-z$. T. mit der Folge mangeInder Kooperation und unbefriedigenden Informationsaustausches. Aus diesen Gründen ist es verständlich, dass viele Betroffene verunsichert sind und eine zweite Meinung suchen und sich jemanden wünschen, der sie emotional unterstützt und Halt gibt.

In der vorliegenden Arbeit sollte ein Expertenforum im Internet zum Thema „ungewollte Kinderlosigkeit" unter folgenden Fragen untersucht werden.

1. Welche Erfahrungen haben Frauen und Männer, die sich an ein entsprechendes Expertenforum wenden, mit der medizinischen Betreuung und Behandlung bei Kinderlosigkeit? Worin liegt ihr größter Wissensbedarf? Welche Schwachstellen der medizinischen Versorgung lassen sich daraus auf der inhaltlichen und der Beziehungsebene erkennen?

2. Wie häufig und von wem wird ein spezifisches Internetangebot genutzt? Wie sehen der Inhalt und die Form der Fragen aus? Sind die Nutzer mit dem Angebot der Seite zufrieden? Damit soll das betreffende Internetangebot hinsichtlich seiner Funktion (additiv und/oder konkurrierend zur traditionellen Medizin), seiner Qualität (aus Patientensicht) und seines Bedarfs (Zukunftsmedizin) bewertet werden.

3. Da bisher kaum Analysen zu Expertenforen im Internet vorliegen, ist auch die methodische Frage von Bedeutung, wie sich die unter 1. und 2. genannten Aspekte am besten aufarbeiten lassen. Ist z. B. die methodische Triangulation (d. h. in diesem Fall die methodische Vielfalt in Form retrospektiver Analyse bisheriger Fragen, prospektiver Erhebung zu der Bewertung eines aktuellen Inter- 
netangebots und der Verknüpfung von Inhaltsanalyse und Befragung) geeignet, die Fragestellung zu bearbeiten? 


\section{Methoden}

Für die Fragestellung und Ziele der Untersuchung erschien eine Kombination mehrerer Erhebungsstrategien und Auswertungsverfahren als sinnvoll:

1. Retrospektive Auswertung der bisherigen Anfragen an das Expertenforum „Kinderwunsch" der Website www.rund-ums-baby.de (2003, Abbildung 1) mit dem Ziel, die Anfragen nach Inhalt (gegebenenfalls auch nach indirekten Mitteilungen) zu klassifizieren.

2. Online-Befragung der Nutzer des Expertenforums mit dem Ziel, Gründe für den Besuch des Forums zu erheben, ein Nutzerprofil zu skizzieren und die Qualität des Expertenforums bewerten zu lassen.

3. Verknüpfung der Anfragen der unter Punkt 2. befragten Nutzer mit ihren Antworten auf die Online-Befragung, um mögliche Zusammenhänge zwischen den Gründen für den Besuch des Expertenforums und dem Nutzerprofil zu finden.

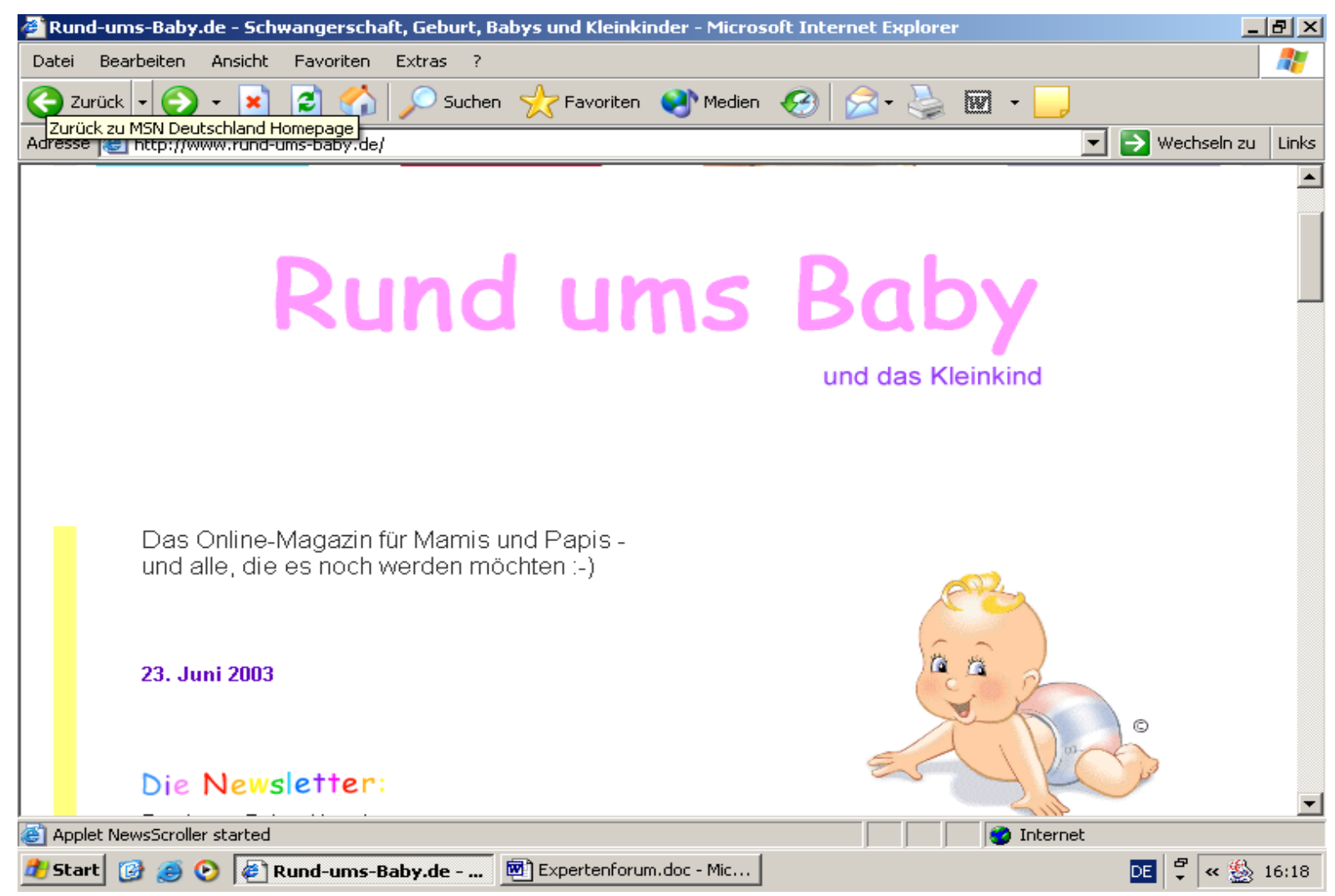

Abbildung 1: Die Website www.rund-ums-baby.de, 2003 
Die unter Punkt 2 und 3 genannte Befragung wurde in die Website „rund-umsbaby.de - Onlinemagazin für Mamis und Papis und alle, die es noch werden möchten" integriert (Abbildung 1).

Die Seite ist über die üblichen Suchmaschinen (GOOGLE, YAHOO!, FIREBALL u.a.) mit Schlagwörtern wie „Unfruchtbarkeit“, „Sterilität“, „Infertilität“, „Kinderlosigkeit“, „Kinderwunsch“ u.ä. zu erreichen. Die User finden auf der Website Informationen über Kindererziehung und -pflege, Links zu anderen Websites, Adressen von Beratungseinrichtungen, sozialrechtliche Hinweise, Literaturtips usw. Themenspezifische Chatrooms (z. B. „Rund ums Baby“; „Schwanger - wer noch?“; „Kinderwunsch“) erleichtern den direkten Austausch der Besucher. In verschiedenen Foren (z. B. „Geburt und Nachsorge“; „Mütterberatung“; „Verhütung“) beantworten Experten in ihrem Fachgebiet Fragen der Besucher.

Eines dieser Foren ist das Kinderwunsch-Expertenforum. Besucher können sich mit Fragen an verschiedene Experten mit unterschiedlichen Zuständigkeitsbereichen wenden (Abbildung 2).

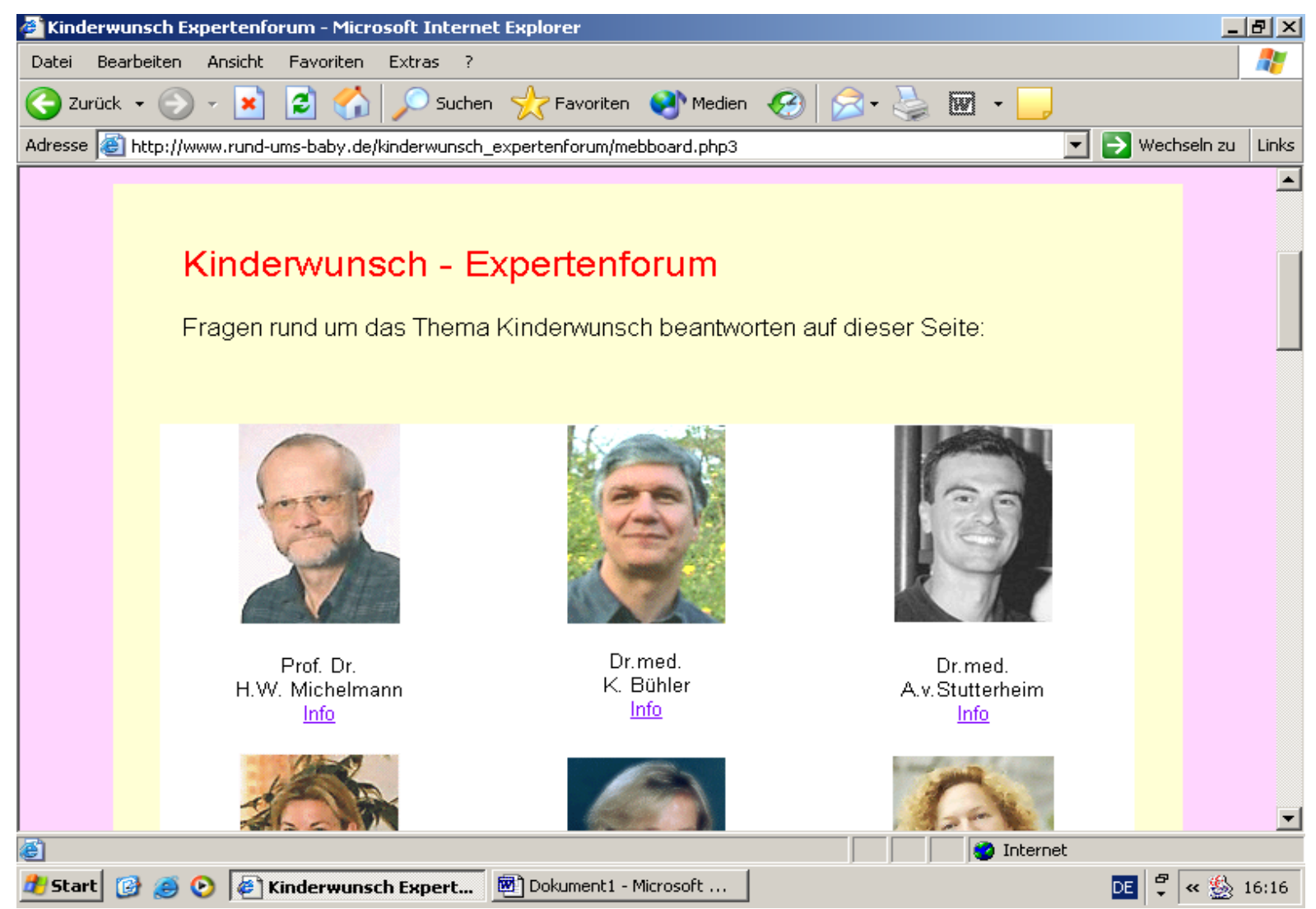

Abbildung 2: Expertenforum der Seite „rund-ums-baby.de“, 2003 
Es werden fachspezifische Fragen aus folgenden Bereichen beantwortet:

- gynäkologische Probleme

- Probleme in der Schwangerschaft

- andrologische und laborrelevante Fragestellungen

- Fragen zur Endokrinologie

- Ernährung

- Reproduktionsmedizin.

Alle Informationen sind unverbindlich und allgemeiner Natur. Es werden keine Ferndiagnosen gestellt; gegebenenfalls empfehlen die Experten die (erneute) Konsultation beim Facharzt.

\subsection{Retrospektive Auswertung}

\subsubsection{Vorüberlegung}

Der Hilfebedarf ungewollt kinderloser Paare sollte in einem ersten Schritt durch eine qualitative Datenanalyse erschlossen werden. Analysiert wurden alle Fragen an die Experten des Forums „www.rund-ums-baby.de“ im Zeitraum zwischen dem 28.07.1999 und dem 17.02.2001.

Diese Art der Datenerhebung lässt sich als indirekte Beobachtung beschreiben, eine Form der non-reaktiven Methode der Sozialforschung (Berg B 1989). Aus der Analyse der gesammelten Fragen sollte indirekt auf die Betreuungs- und Bedürfnissituation der Kinderwunschpaare geschlossen werden.

Vorteile einer qualitativen Studie ergeben sich durch das Herausarbeiten von mehr Details als bei der quantitativen Umfrage. Die qualitative Auswertung ermöglicht die Berücksichtigung des inhaltlichen Reichtums („Tönung“) der individuellen Fragen.

\subsubsection{Stichprobe}

Im Zeitraum zwischen dem 28.07.1999 und dem 17.02.2001 gab es 10.000 Einträge im Expertenforum auf der Internetseite „www.rund-ums-baby.de“. Diese Einträge 
setzten sich aus Fragen an die Experten, deren Antworten und Kommentaren der Besucher zusammen. Aus dieser Datenmenge von 10.000 Einträgen konnten 3.840 Einträge eindeutig als Anfragen an die Experten klassifiziert und extrahiert werden. Bei den restlichen 6.160 Einträgen handelte es sich um (Ausschlusskriterien):

- Antworten der Experten,

- Wiederholte Fragen von einer Person;

- Spezielle Fragen zur länger bestehenden Schwangerschaft, die auf natürlichem Weg entstanden ist;

- Gynäkologische Fragestellungen, die nicht direkt mit einem Kinderwunsch korrelierten;

- Fragen zu Verhütungsmethoden;

- Anfragen, die nicht direkt an das Expertenforum gerichtet waren;

- Persönliche Fragen an die Experten.

Zur gesonderten Analyse wurden sämtliche Fragen von Männern, soweit sie als diese identifizierbar waren, separat gesammelt und vollständig ausgewertet (Totalerhebung von $\mathrm{N}=69$ ). Die Anfragen der Frauen wurden von 1 bis 3771 nummeriert. Daraus wurde mittels Zufallszahlen (SAS-Procedure normal) eine Stichprobe ( $\mathrm{N}=245)$ gezogen. Die ausgewählten Fragen der Frauen und sämtliche Fragen der Männer wurden zur qualitativen Auswertung in das Analyse-Programm Atlas.ti eingelesen.

\subsection{Befragung der Nutzer}

\subsubsection{Vorüberlegung}

Zur Erhebung eines Überblicks über die derzeitige Betreuungssituation ungewollt kinderloser Paare in Deutschland wurde die standardisierte Online-Befragung als noch junge Methode der quantitativen empirischen Sozialforschung gewählt. Die Methode fußt auf der etablierten Technik der schriftlichen Befragung; neuartig ist die Übermittlung des Fragebogens an den Befragten über das Internet.

Richtlinien für die Konzeption und Durchführung von Online-Erhebungen gründen vor allem auf den praktischen Erfahrungen der Markt- und Meinungsforschung und der 
Diskussion über die Güte kommerzieller Datenerhebungen (Arbeitskreis Deutscher Markt- und Sozialforschung, ADM et al. 2001).

Die Ergebnisse einer Online-Befragung unterliegen hinsichtlich der Repräsentativität und Selektivität den gleichen Fehlerquellen wie andere Methoden der quantitativen empirischen Umfrageforschung, z. B. schriftliche oder telefonische Befragung. Einige Besonderheiten sind zu berücksichtigen:

- Aufgrund der angewandten Methode können nur ungewollt kinderlose Paare in Haushalten (ggf. auch in Betrieben) mit Internetanschluss einbezogen werden. Die Untersuchungsergebnisse sind dann nur bedingt auf die Gesamtheit der ungewollt kinderlosen Paare übertragbar.

- Es besteht die Gefahr der Stichprobenverzerrung, da bestimmte Bevölkerungsgruppen und -schichten (Akademiker, Arbeitnehmer) häufiger über Internetzugang verfügen (Selbstselektion der Stichprobe).

- Repräsentativität kann bei Online-Befragungen nur hinsichtlich der Nutzer eines bestimmten Online-Angebotes (in diesem Fall der Nutzer der Website „rund-umsbaby.de") erreicht werden. Generalisierbare Aussagen über alle kinderlosen Paare wären auf Grundlage der Online-Befragung nur möglich, wenn die Ausstattung der Haushalte mit einem Internetanschluss nachweislich weit fortgeschritten ist (ähnlich der Telefonanschlüsse privater Haushalte) und die Risikogruppe bzw. der Adressatenkreis das Angebot auch unterschiedslos nutzen würde.

- Schwierigkeiten beim Rekrutieren der Studienteilnehmer können auch dadurch auftreten, dass zumindest einigen Teilnehmern an der Online-Befragung während der Beantwortung Verbindungsgebühren entstehen.

\subsubsection{Stichprobe}

Die Feldzeit sollte so bemessen sein, dass 1. alle zur Zielgruppe gehörenden Personen eine Chance hatten, an der Befragung teilzunehmen, und 2. die Stichprobe statistisch aussagefähig ist. 
Zu 1) Zu kurze Feldzeiten führen in der Regel zu einer Stichprobenverzerrung, da Personen, die das Internet nur selten nutzen, eine geringere Auswahlwahrscheinlichkeit haben.

Zu 2) Als Zielkriterium für den Stichprobenumfang wurde die Frage 9 (Anhang S. 90) gewählt (erste Anlaufstelle bei Fragen zur Kinderlosigkeit). Wir gingen aufgrund einer Vorerhebung davon aus, dass ca. 10\% der Besucher des Expertenforums das Forum als erste Anlaufstelle wählten. Die Abschätzung dieses Anteils sollte mit einer Genauigkeit von +/- 3 \% (entspricht, bezogen auf 10 \% "Prävalenz", einer Genauigkeit von $30 \%[0,3])$. Die Sicherheit der Schätzung sollte $95 \%$ betragen. Auf Basis dieser Annahme und Vorgaben war eine Stichprobengröße von mindestens $N=390$ erforderlich. Die Berechnung erfolgte mit den Sample Size Tables for Clinical Studies (Machin et al. 1997, S. 140-142).

\subsubsection{Fragebogen}

\subsubsection{Entwicklung}

Der Online-Fragebogen wurde auf der Basis der Fragestellungen und Ziele entwickelt. Vorläufer-Versionen wurden im Doktoranden-Seminar der Abteilung Allgemeinmedizin und in kleinen Arbeitsgruppen - mit einem Experten des Expertenforums (Prof. Michelmann) - präsentiert, diskutiert und verändert.

\subsubsection{Aufbau}

Die zwei wichtigsten Anforderungen an den Fragebogen waren eine differenzierte Datenerhebung und eine möglichst geringe Belastung der Studienteilnehmer durch komplizierte Fragen und Antwortschemata, um die Abbruchrate niedrig zu halten. Der Online-Fragebogen sollte auch für unerfahrene Internetnutzer zu handhaben sein. Deshalb wurden optisch hervorgehobene Buttons und vordefinierte Eingabefelder genutzt.

Die automatische Filterführung (Filterfragen) realisierte, dass dem jeweiligen Befragten auch wirklich nur die Fragen präsentiert wurden, die für inn zutreffend waren. 
Dadurch erschien der Fragebogen subjektiv kürzer; fehlerhafte, unlogische Antworten ließen sich reduzieren.

Beim Aufbau des Fragebogens wurden die „allgemeinen Regeln für die Fragebogenkonstruktion“ (Fuchs 1994, S. 129) berücksichtigt:

- Besonderes sorgfältiges Erarbeiten der Einleitung des Fragebogens sowie der ersten Fragen, da hier die Abbruchswahrscheinlichkeit am höchsten ist;

- Platzieren der inhaltlich wichtigsten Fragen im zweiten Drittel des Fragebogens;

- Fragen zur Sozialstatistik wurden am Schluss der Befragung gestellt;

- Die Filterführung des Fragebogens wurde auf vollständig enthaltene Befragungsvarianten geprüft.

\subsubsection{Vorstellen der Studie}

In einem kurzen einleitenden Text (Anhang 8.1.1) wurden die Studie und ihre Ziele vorgestellt. Es wurde deutlich darauf hingewiesen, dass die Anonymität der Studienteilnehmer gewahrt bleibt und dass die Nicht-Beantwortung des Fragebogens keine negativen Auswirkungen auf die Antwortbereitschaft der Experten hat. Des Weiteren erfuhren die Befragten die E-Mail-Adressen und Anschriften der mitwirkenden Abteilungen (Abteilung Allgemeinmedizin und Frauenklinik der Universität Göttingen), um sich ggf. über die Befragung direkt zu informieren oder auch beschweren zu können. Über diese Adressen hatten die Befragten zugleich die Möglichkeit, bei inhaltlichen oder technischen Problemen mit dem Ausfüllen des Fragebogens Kontakt mit den Verantwortlichen aufzunehmen. Den Interessierten wurde ebenfalls durch Links die Möglichkeit geboten, auf andere themenbezogene Seiten zu gelangen. Auf diese Weise sollte eine gute Resonanz erzielt und zur Teilnahme an der Studie motiviert werden. 


\subsubsection{Selektion der Studienteilnehmer}

Die erste Frage des Fragebogens diente als Filter, um Erstteilnehmer von wiederholten Besuchern bzw. Studienteilnehmern und Besuchern, die keine Frage zum Thema Kinderwunsch haben, zu trennen:

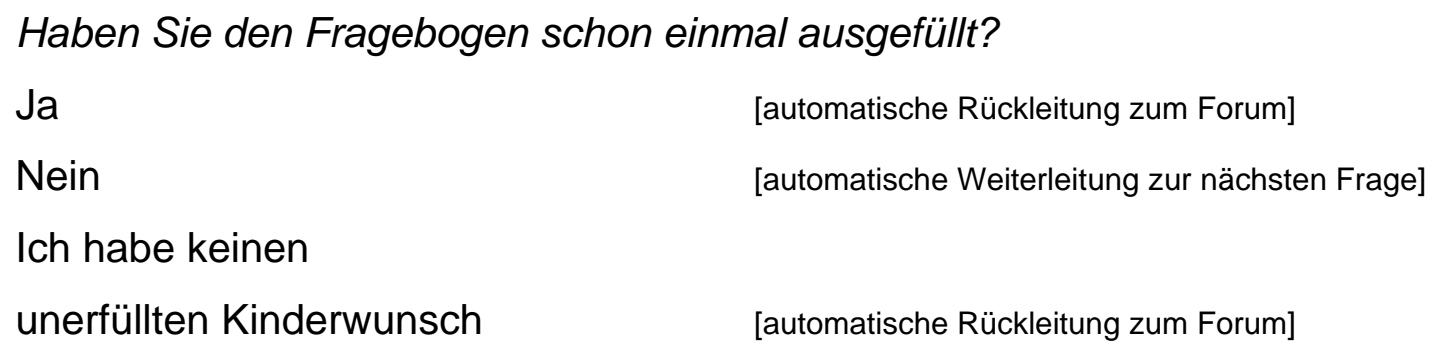

Die erste und die letzte Gruppe (wiederholte Fragesteller und Fragesteller, die keine Frage zum Thema Kinderwunsch hatten) wurden auf eine Seite geleitet, auf der nochmals darauf hingewiesen wurde, dass ihre Frage an die Forumexperten schon abgesandt wurde und umgehend von dem gewünschten Fachmann beantwortet wird. Auf diesem Weg wollten wir Mehrfachteilnahmen (und Verärgerung bei den Nutzern) vermeiden.

Um die Freiwilligkeit der Studienteilnahme sicherzustellen und gegenüber den Nutzern zu verdeutlichen, wurde explizit gefragt, ob der Fragesteller bereit ist, an der Studie teilzunehmen.

Würden Sie an der Studie teilnehmen und den Fragebogen ausfüllen? $\mathrm{Ja}$ [automatische Weiterleitung zum Fragebogen]

Nein [automatische Rückleitung zum Forum]

Bei Bejahung der Frage wurde der Studienteilnehmer sofort zum eigentlichen Fragebogen weitergeleitet; bei Verneinung erschien die oben erwähnte Seite. Die Befragten hatten im weiteren Verlauf des Fragebogens zu jedem Zeitpunkt die Möglichkeit, das Interview abzubrechen. 


\subsubsection{Fragen zum Besuch des Expertenforums}

Der erste Fragenabschnitt diente der Darstellung der Gründe für den Besuch des Expertenforums: wie und über welches Medium das Expertenforum erreicht wurde und wie man von diesem Forum erfahren hatte.

Die Antwort auf die dritte Frage („Stellen Sie heute zum ersten Mal eine Frage an das Expertenforum?") unterteilte die Studienteilnehmer in zwei Gruppen (Filterfrage). Im sich anschließenden ersten großen Fragenkomplex wurde nur die Gruppe, die häufiger das Forum nutzte, befragt: zur Zufriedenheit mit den Informationen, welche die Experten liefern, und zu Wünschen an das Expertenforum. Es wurde exploriert, ob die Diskussion und die Antworten der Experten in ein späteres Arzt-PatientGespräch eingebracht und ob aufgrund der Expertenempfehlung der Arzt gewechselt oder ein Arzt bzw. eine Spezialklinik aufgesucht wurden. Danach lief der Fragebogen für alle Befragten analog weiter.

\subsubsection{Derzeitige Versorgungssituation}

Die zweite Frageneinheit diente der genaueren Orientierung über die derzeitige ärztliche Versorgungssituation der Befragten. Eine Filterfrage (,Sind Sie in Behandlung?") trennte die Gruppe der in Behandlung befindlichen Patienten von den übrigen Befragten.

Es folgten Fragen nach der Dauer und Art der Behandlung. Daran anschließend wurden die Studienteilnehmer gebeten, die Betreuung durch die verschiedenen in die Therapie involvierten Ärzte, sowie die Aufklärung und Problemdiskussion durch die Ärzte zu bewerten. Das Antwortformat war eine 4-stufige Likert-Skala.

\subsubsection{Nutzerprofil}

Sozialstatistische Fragen zu Alter, Geschlecht, Größe des Wohnorts, Familienstand und Ausbildung standen am Ende des Fragebogens. 


\subsubsection{Technische Präsentation}

Ähnlich der schriftlichen Befragung stellt auch die Online-Befragung hohe methodische Anforderungen, weil die Befragten sich ohne Hilfe eines Interviewers im Fragebogen zurechtfinden, die Fragen verstehen und adäquat beantworten müssen. An die Programmierung und die Gestaltung des Fragebogens wurde zusätzlich die Anforderung gestellt, dass der Fragebogen von unterschiedlichen Betriebssystemen sowie Browserfabrikaten und -versionen problemlos dargestellt und bearbeitet werden kann.

Der Fragebogen sollte sich jedes Mal öffnen, nachdem eine Frage an einen Experten des Forums gerichtet wurde („Popping-Up“).

Aus Kosten- und Aufwandsgründen wurde auf die Vergabe von Anreizen (,Incentives“) für die Teilnahme verzichtet. Eine kurze möglichst präzise Aufklärung über den Nutzen der Studie sollte einen „neutralen“ Anreiz für die Teilnahme schaffen, damit durch Art und Umfang der Incentives die Stichprobe nicht verzerrt wird.

In der Online-Befragung sollten nur ungewollt kinderlose Paare teilnehmen. Die zielgruppenspezifische Ansprache wurde realisiert, indem der Fragebogen nur dann aktiviert wurde, wenn ein Besucher der Website seine Frage an das KinderwunschExpertenforum richtete. Der Fragenbogen selbst konnte nicht über Links oder Suchmaschinen geöffnet werden.

\subsection{Pilotphase}

Vor Beginn der Befragung wurde der Fragebogen in einer 72-stündigen Pilotphase getestet. Der Test bezog sich auf Verständlichkeit und Praktikabilität dieser Art der Befragung (z. B. Kompatibilität zu den gängigen Browsern). Auch die Teilnehmerquote („Rücklauf“) sollte geprüft werden.

In einer abschließenden Frage während der Pilotphase (Anhang 8.1.3, Frage 23) ging es um mögliche Verständnisschwierigkeiten und technische Probleme beim Lesen und Beantworten („Anklicken“) des Fragebogens. Die Zusatzfrage wurde offen 
formuliert, damit der Fragebogen nicht zu lang und zeitraubend wird und die Testteilnehmer aus finanziellen Erwägungen die Befragung vorzeitig abbrechen.

Die Ergebnisse der Pilotphase sind in Kurzform in Kapitel 5.1 („Auswertung der Pilotphase") dargestellt.

\subsection{Durchführung der Untersuchung}

Die Online-Befragung dauerte vom 26.08.2001 bis zum 28.02.2002. Die Feldzeit wurde so bemessen, dass zum einen alle zur Zielgruppe gehörenden Personen eine Chance hatten, an der Befragung teilzunehmen, und zum anderen eine repräsentative Stichprobengröße potenziell realisierbar war.

Während der Feldphase hat die Autorin jede Woche einen Fragebogen ausgefüllt und abgesendet, um eventuell auftretende Fehler in der Programmierung oder Schwierigkeiten mit dem Browser zu entdecken und umgehend zu beheben. Die elektronisch eingegebenen Antworten wurden zusätzlich per Hand notiert, um die entsprechenden Fragebögen später in der Datenbank des Servers zu identifizieren und auf Übereinstimmung zu vergleichen. Diese Fragebögen wurden anschließend aus dem Datensatz entfernt.

Vor der Feldphase wurde ein Kode-Plan (Tabelle 5; S. 44) mit Definition der Variablen und ihre möglichen Ausprägungen entwickelt. Die Daten konnten während der Online-Befragung in Abständen auf die Datenbank der Abteilung Allgemeinmedizin übertragen und direkt in ein Datenanalyse-Programm eingelesen werden, um Qualitätskontrollen zu ermöglichen.

\subsection{Datenschutz}

Datenschutz und Datensicherheit orientierten sich streng am Protokoll des ADM (2001). Die erhobenen Daten wurden auf dem Server der Website „rund-umsbaby.de" temporär gespeichert. Der Webmaster der Seite stellte sicher, dass unbefugte Dritte keinen Zugriff auf die Daten nehmen konnten.

Mehrfach während der Befragung und nach Abschluss der Befragung wurden die Daten in die Abteilung Allgemeinmedizin verschlüsselt übermittelt, so dass kein Drit- 
ter den Datentransfer einsehen konnte. Die Datenbank wurde durch ein Passwort geschützt. Die persönlichen Daten der Studienteilnehmer (Name, E-Mail-Adresse) wurden unmittelbar nach Eingang der Daten in die Abteilung Allgemeinmedizin von den Befragungsdaten und Fragen an die Experten getrennt. Die Zuordnung der Fragebogen-Antworten und der Anfrage an das Expertenforum war nur noch über eine gemeinsame Kodenummer möglich. Die Adressdaten der Teilnehmer wurden gelöscht; die Kode-Liste wurde verschlossen.

\subsection{Auswertung}

Die durch verschiedene Erhebungsmethoden gewonnenen Daten wurden - im Sinne einer methodischen Triangulation - kombiniert bzw. verknüpft. Triangulation wird in der sozialwissenschaftlichen Literatur als „die Kombination von Methodologien bei der Untersuchung desselben Phänomens“ (Flick 1995, S. 432) interpretiert, um einen empirischen Gegenstand angemessen, d.h. valider zu erfassen. Das Potenzial einer solchen Methodenkombination liegt insbesondere darin, „unterschiedliche Perspektiven zu verbinden und möglichst unterschiedliche Aspekte des untersuchten Gegenstandes zu thematisieren“ (Flick 1995, S. 433).

Die Methodische Triangulation unterteilt sich in zwei Formen (Jakob 2001):

- Within-method: Kombination unterschiedlicher Auswertungsverfahren, die auf einen Datensatz Anwendung finden;

- Across-method:Kombination verschiedener Forschungs- bzw. Erhebungsmethoden, um ein empirisches Phänomen zu untersuchen.

Von besonderer Bedeutung für die Triangulation ist die Integration quantitativer und qualitativer Methoden (Prein et al. 1993). Hierbei lassen sich drei Ansätze unterscheiden:

- Phasenmodell:

Hier werden zuerst qualitative Verfahren angewandt, um anschließend - und insofern chronologisch - die hieraus gewonnenen Hypothesen im Rahmen einer Hypothesenüberprüfung mittels standardisierter Verfahren zu überprüfen; 
- Konvergenzmodell:

In diesem Modell dienen die gewonnenen Daten zur gegenseitigen Validierung, wobei beide Ansätze als adäquat und in diesem Sinne gleichberechtigt angesehen werden;

- Komplementaritätsmodell:

Hier beziehen sich die jeweiligen Methoden auf unterschiedliche Gegenstandsbereiche, so dass sie sich in besonderer Weise ergänzen.

Aufbauend auf den obigen Erkenntnissen wurde eine Across-method-Triangulation im Sinne des Komplementaritätsmodells durchgeführt. Im ersten Schritt wurden die an das Expertenforum gestellten Fragen inhaltsanalytisch ausgewertet. Hierzu wurden verschiedene Verfahren der Inhaltsanalyse angewendet und miteinander kombiniert. Die so gewonnenen Ergebnisse wurden danach anhand des quantitativen Datenmaterials, welches aus der Internetbefragung gewonnen wurde, überprüft und anschließend wieder am qualitativen Material "gespiegelt“. Im dritten Auswertungsschritt wurden Einzelfälle auf Basis der an das Expertenforum gestellten Fragen und den Antworten auf den Fragebogen analysiert. Die Komplexität der Erwartungen an das Expertenforum und der Beratungsbedarf sollte auf diese Weise dargestellt werden.

\subsubsection{Quantitative Auswertung}

Zur statistischen Auswertung wurden die elektronisch gespeicherten Daten als comma seperated values (csv-Format) in SAS, Version 8.1 eingelesen. Aufgrund der explorativen Fragestellung beschränkte sich die Auswertung auf Häufigkeitsauszählungen, Mittelwertsberechnungen und deskriptive Vergleiche mit den freq- und means-Prozeduren (Machin et al. 1997).

\subsubsection{Qualitative Auswertung}

Analysiert wurde das Datenmaterial (Fragen an das Expertenforum) durch die Kodierung einzelner Textteile (d.h. die Zuordnung der Kodierungseinheit zu den verschiedenen Kategorien). Kodierungseinheiten (Quotations) können ein Wort, Satz oder ganze Absätze sein. Die Kodes können anschließend in Kode-Familien zusammen- 
gefasst werden. Die Fragen an das Expertenforum wurden gegliedert und ihre Themen („Grundideen“) herausgearbeitet, um die Gedankens- und Erlebenswelt der Befragten besser zu verstehen (Bortz und Döring 2002, S. 329). Diese Hintergrundstrukturen wurden dann verglichen. Es wurde nach Ähnlichkeiten und Unterschieden gesucht und es wurde versucht Muster, soweit vorhanden, zu erkennen. Verbindungen zwischen Kategorien wurden erstellt und es wurde ein Verstehen der emotionalen Reaktionen hinsichtlich der Unfruchtbarkeit ebenso die erleichternden und erschwerenden Faktoren herausgearbeitet.

Die Gütekriterien quantitativer Forschung, wie Objektivität, Reliabilität und Validität wurden in der qualitativen Analyse der Texte in modifizierter Form verwendet. Dabei spielen die Begriffe "Objektivität" und „Reliabilität" eine eher untergeordnete Rolle; größere Bedeutung wurde den verschiedenen Kriterien der Validität beigemessen (Bortz und Döring 2002, S. 327-328).

Zur Standardisierung der qualitativen Auswertung wurde vor der Datenanalyse ein Kategorienraster entwickelt (deduktives Verfahren). Das Kategorienschema lehnt sich an die Fragestellungen, Hypothesen und den Fragebogen an. Die genaue Definition der Kategorien sollte die Objektivität der Studie fördern. Anker-Beispiele und genaue Kodierregeln sollten eine eindeutige Zuordnung bei Abgrenzungsproblemen zwischen den Kategorien gewährleisten (Mayring 1988, S. 75). Im Verlauf der Datenanalyse wurde das Kategorienraster durch die Bildung neuer Kategorien und Subkategorien ergänzt und verfeinert (induktives Verfahren).

Zur Sicherung der Intrakodier-Reliabilität wurden in allen Phasen der Analyse einheitliche Kriterien für die Zuordnung der Analyseeinheiten und Kategorien verwendet. Die Validität wurde gewährleistet durch wiederholte Arbeitsbesprechungen mit anderen am Projekt beteiligten Forschern und außenstehenden Kollegen (argumentative und konsensuelle Validierung). Zur Sicherung der Validität diente auch die methodologische Triangulation (Flick 1995, S. 432-434; Denzin 1989), um ein genaueres Bild von der Situation ungewollt kinderloser Paare zu erhalten und unterschiedliche Aspekte zu thematisieren. 
Ausgewertet wurden die Einzelanalysen mit inhaltsanalytischen Methoden in Atlas.ti, wie oben dargestellt. Atlas.ti ist ein Softwareprogramm zur Durchführung von qualitativen Analysen umfangreicher Texte sowie Daten in Grafik-, Audio- und Videoformat.

Bei der Arbeit mit Atlas.ti werden alle für ein Projekt bedeutsame Dokumente (Text, Bild oder Multimedia) in einer "Hermeneutischen Einheit" (Hermeneutic Unit) zusammengefasst. Dort werden auch sämtliche späteren Auswertungsschritte, d.h. alle Kodierungen der Texte, Relationen, Kommentare in Form einer Hypertextstruktur gespeichert.

Auf der Ebene der Primärdokumente werden relevante Text- oder Bildausschnitte markiert und mit Kommentaren versehen. Zusätzlich stehen dem Nutzer Werkzeuge zur Verfügung, um die Kodes (Schlagwörter, Konzepte) miteinander zu vernetzen, die erzeugten Relationen zu benennen und Begriffsnetze bzw. Modelle zu visualisieren. Die so erzeugten Modelle erlauben es, von zusammenfassenden und abstrahierenden Konzepten aus bei Bedarf jederzeit auf die innen zugrunde liegenden Textpassagen in ihrem Ursprungskontext zurückzugreifen (Muhr 1997, Thinktool 2002).

Wenn im Ergebnis-Kapitel Zitate wiedergegeben werden, wurden zur besseren Lesbarkeit unbedeutende Rechtschreibfehler (z. B. typische „Verdreher") in den Zitaten korrigiert und von den Fragestellern genutzte Abkürzungen in eckigen Klammern erklärt.

\subsubsection{Verknüpfung von selektiven Einzelfallanalysen und quantitativem Da- tenmaterial}

Während der Datenspeicherung wurden Fragebogen und die dazugehörige Anfrage mit einem Zahlenkode versehen, so dass man in der Auswertungsphase jeder Anfrage den passenden Fragebogen zuordnen konnte („matching“). So war es möglich, Zusammenhänge zwischen der Anfrage und dem Nutzerprofil und somit ein Betreuungs- bzw. Informationsdefizit zu erkennen.

In den Einzelanalysen wurden besonders interessante „Fälle" inhaltsanalytisch ausgewertet. Dabei sollten die Fragen an das Expertenforum nicht auf die vorab definierten Dimensionen (Variablen) beschränkt werden (siehe 4.6.2), sondern exemplarisch 
sollte ein reichhaltigeres Bild über die Situation ungewollt kinderloser Paare, die sich an das Expertenforum wenden, entstehen.

Die Personen für die Einzelanalysen wurden so gewählt, dass sie „hinsichtlich einer gleichen oder ähnlich strukturierten größeren Menge von Phänomen [Kategorien] als typische Fälle oder besonders prägnante oder aussagekräftige Beispiele gelten“ (Hartfield 1982, S. 160): Es wurde eine kleine Anzahl besonders kritischer und/oder stark emotional geprägter Anfragen, die einen hohen Leidensdruck erkennen lassen, herausgesucht und auf die dazugehörigen Antworten auf den Fragebogen bezogen. Der darin sich (möglicherweise) äußernde Beratungsbedarf sollte konkretisiert und ggf. sollten Hypothesen für eine zukünftige Erhebung über solche Betreuungsdefizite formuliert werden. 


\section{Ergebnisse}

Im ersten Abschnitt (5.1) werden die Ergebnisse der Pilotphase vorgestellt. In ausführlicher Darstellung folgen die Ergebnisse der Internetbefragung (5.2) und der Inhaltsanalyse der Anfragen an das Expertenforum (5.3).

\subsection{Auswertung der Pilotphase}

In der 72-stündigen Pilotphase war der Fragebogen probeweise ins Internet gestellt worden. Er wurde bei jeder Frage an das Expertenforum der Seite www.rund-umsbaby.de aktiviert

In der Pilotphase wurde der Fragebogen insgesamt 35 Personen präsentiert. Von diesen hatten sechs Personen das Expertenforum während der Pilotphase offensichtlich schon einmal besucht, denn sie gaben an, den Fragebogen schon ausgefüllt zu haben (siehe 5.2.1); zwei Befragte hatten „keine Frage zum Thema Kinderwunsch". Von den verbliebenen 27 Befragten verweigerten drei Personen die Teilnahme. Daraus ergibt sich für die Pilot-Studie eine Rücklaufquote von $89 \%$.

Keiner der Befragten berichtete von technischen Problemen bei der Präsentation und beim Ausfüllen des Fragebogens. Die abschließende, offene Frage nach der Verständlichkeit und Praktikabilität des Fragebogens beantwortete die Mehrzahl der Befragten positiv oder ohne (negativen) Kommentar. Ein Studienteilnehmer hatte Probleme bei einer Frage, die daraufhin präzisiert wurde (Anhang 8.1.3, Frage 22).

In der Pilotphase gab es ein technisches Problem bei der Speicherung und Verarbeitung der Daten. Die Kodierung und Programmierung des Fragebogens wurden daraufhin geändert.

\section{$5.2 \quad$ Internet-Befragung}

\subsubsection{Ausschöpfung der Stichprobe}

Der Fragebogen war vom 27.08.2001 bis 28.02.2002 im Expertenforum der Website „rund-ums-baby“ präsent. In dieser Zeit wurde der Fragebogen insgesamt 1.305-mal aufgerufen. Davon gaben 632 Befragte $(48,4 \%)$ an, den Fragebogen schon ausge- 
füllt zu haben; 53 Befragte (4,1\%) hatten nach eigener Angabe keinen Kinderwunsch. Von den verbleibenden 620 Befragten, die den Fragebogen noch nicht ausgefüllt hatten, verweigerten 97 die Teilnahme (Tabelle 1). Zu diesen eindeutigen („harten“) Verweigerern (15,6\%) könnte noch eine unbekannte Anzahl an Personen hinzukommen, die nach Einblenden des Einleitungstextes zum Fragebogen das Internet verlassen haben, ohne den Fragebogen aufzurufen. Ob dies und ggf. wie häufig dies stattfand, ließ sich nicht ermitteln. Bei einem Vergleich zu den monatlichen Anfragen $^{1}$ (Hits) an das Expertenforum, könnte die Zahl dieser Verweigerer im (unwahrscheinlichen) Extrem bei 920 liegen: 2242 Personen haben insgesamt im Untersuchungszeitraum Fragen gestellt (Abbildung 3; grüne bzw. obere Kurve); zu den 1.322 Rücksendungen (blaue bzw. mittlere Kurve) gibt das eine Differenz von 920. Diese eventuellen Verweigerer würden sich zu den 97 „harten“ Verweigern hinzuaddieren. Die rote (untere) Kurve zeigt die pro Monat ausgefüllten Fragebögen.

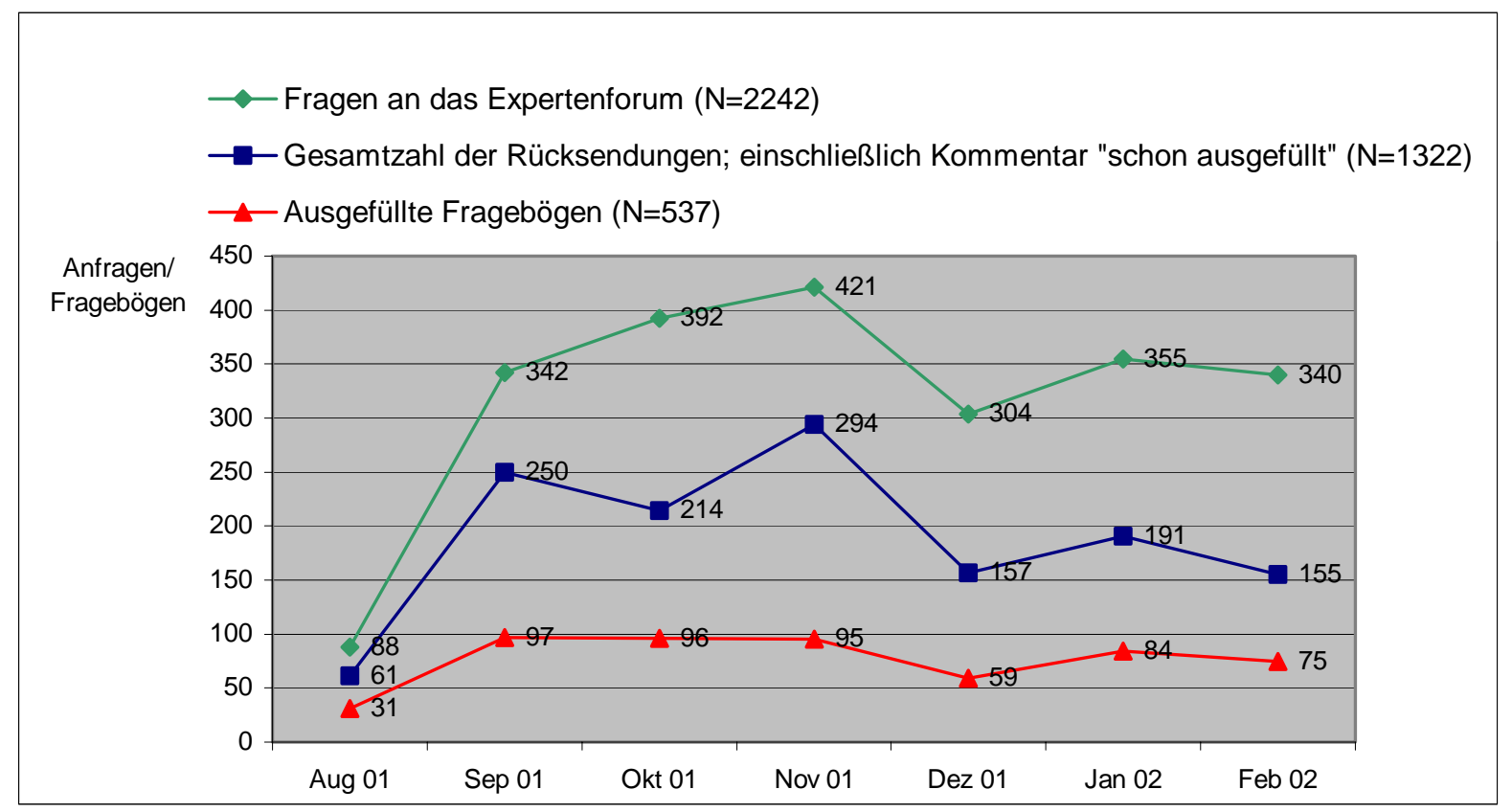

Abbildung 3: Anfragen an das Expertenforum und Fragebogenrücklauf im Untersuchungszeitraum ${ }^{2}$

Auf den ersten Blick auffällig ist die sehr hohe Zahl von Personen, die mitteilten, dass sie den Fragebogen schon ausgefüllt hätten ( $N=632)$, während insgesamt nur 620

\footnotetext{
${ }^{1}$ Der Webmaster der Seite „rund-ums-baby.de“ wurde gebeten, Datum, Uhrzeit und die Anzahl der Anfragen für uns zu dokumentieren (Abbildung 3).

${ }^{2}$ In der Abbildung sind die Anfragen der Autorin, ihre ausgefüllten Fragebögen $(\mathrm{N}=11)$ und unsinnig bzw. doppelt ausgefüllte Fragebögen von Studienteilnehmern $(\mathrm{N}=6)$ mitaufgeführt.
} 
Fragebögen ausgefüllt worden waren. Hierfür gibt es zwei Erklärungen, die sich wechselseitig nicht ausschließen: 1. Einige Befragte gaben fälschlicher Weise an, den Fragebogen ausgefüllt zu haben, weil sie nicht an der Studie teilnehmen oder den Fragebogen einfach "loswerden“ wollten; 2. viele Befragte waren Mehrfachbesucher des Expertenforums, die entweder schon in der Pilotphase den Fragebogen beantwortet hatten oder zum zweiten, vielleicht auch dritten oder vierten Mal während der Feldphase das Expertenforum befragten.

Tabelle 1: Stichprobenausschöpfung

\begin{tabular}{lrr}
\hline & N & $\%$ \\
\hline Aufgerufene Fragebögen insgesamt & 1305 & 100,0 \\
• "Schon ausgefüllte Fragebögen“ & 632 & 48,4 \\
- Keinen Kinderwunsch & 53 & 4,1 \\
Bereinigte Stichprobe & 620 & 100,0 \\
- Verweigerer & 97 & 15,6 \\
& & \\
Studienteilnehmer & 523 & 84,4 \\
\hline
\end{tabular}

\subsubsection{Soziodemographische Merkmale}

Fast alle Studienteilnehmer waren Frauen (496/523). Vier Teilnehmer waren Männer $(0,8 \%) ; 23$ Befragte (7\%) machten keine Angaben zum Geschlecht. Die vollständige Grundauszählung der Befragung ist im Anhang 8.2 dargestellt.

Inwieweit Personen, die sich an das Expertenforum wandten, der Gesamtbevölkerung entsprachen, zeigt Tabelle 2. Die Verteilung nach Geschlecht, Alter, Familienstand und Bildungsniveau der Stichprobe entsprach - erwartungsgemäß - nicht der Gesamtbevölkerung Deutschlands: Nicht nur waren Frauen extrem überrepräsentiert, auch war der Akademikeranteil mit 27,2\% überdurchschnittlich hoch. Singles waren deutlich unterrepräsentiert. 
Tabelle 2: Stichprobe im Vergleich zur Gesamtbevölkerung Deutschlands

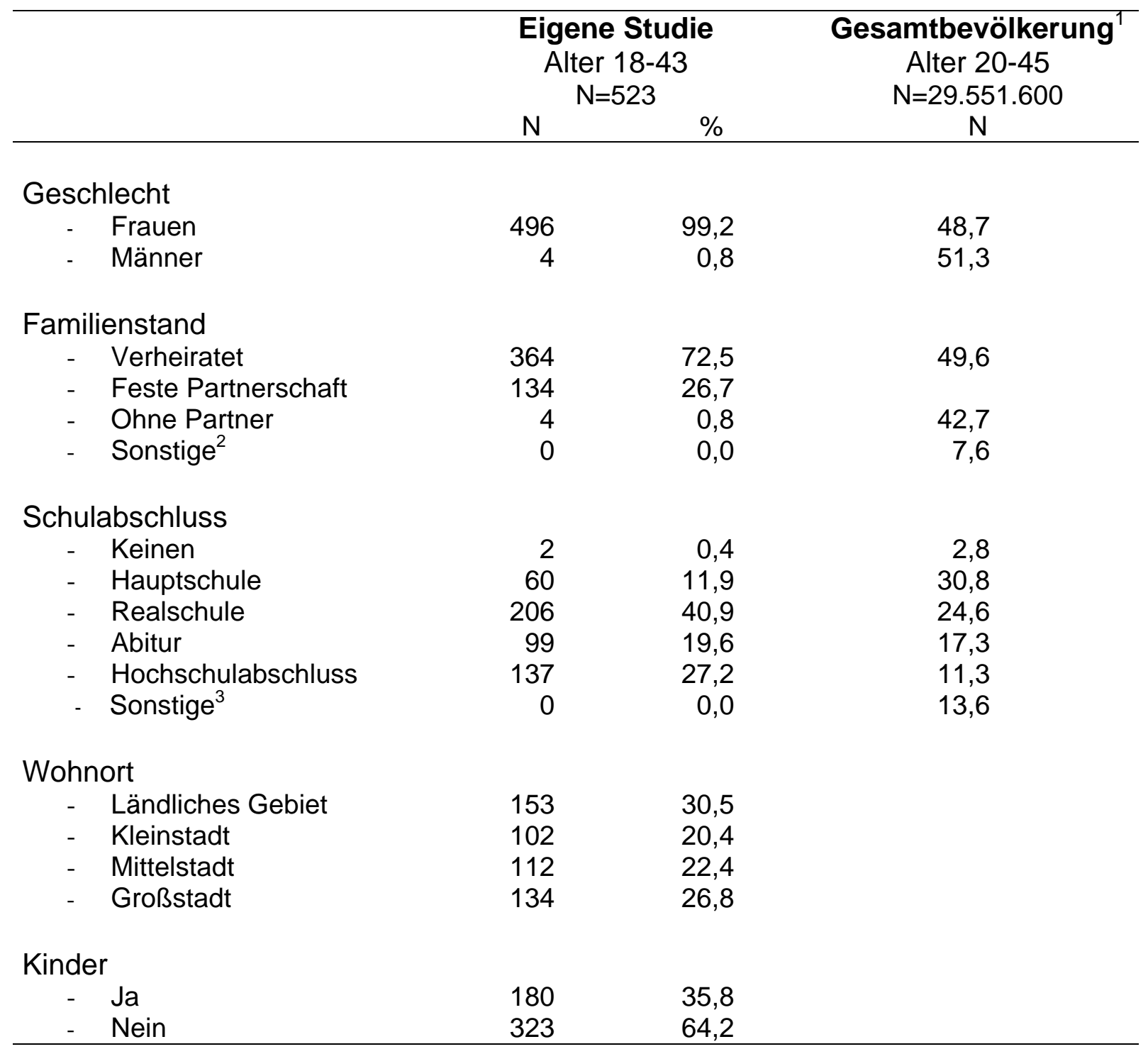

${ }^{1}$ Quelle: Statistisches Bundesamt 2002; eigene Berechnung

${ }^{2}$ sonstiger Familienstand: verwitwet; geschieden

${ }^{3}$ sonstiger Schulabschluss: noch in schulischer bzw. beruflicher Ausbildung; keine Angabe des Abschlusses

\subsubsection{Kontakt mit dem Expertenforum}

Zum Studienzeitpunkt konsultierten 55,1\% (276/501) $)^{1}$ der Befragten zum ersten Mal das Expertenforum; 225 Personen hatten schon häufiger Fragen an das Expertenforum gestellt.

\footnotetext{
${ }^{1}$ Wenn einzelne Fragen nicht von allen Nutzern beantwortet wurden, findet sich in Klammern jeweils die Basis für die Prozentrechnung - in diesem Fall $\mathrm{N}=501$.
} 
Der größte Teil der Befragten (220/474) hatte das Expertenforum durch Zufall im Internet entdeckt; 183 hatten gezielt im Internet nach einer Experten basierten Auskunft gesucht. Von ihrem Arzt oder vom medizinischen Pflegepersonal wurden sechs Personen auf das Expertenforum aufmerksam gemacht.

\subsubsection{Gründe für den Besuch des Expertenforums}

Die meisten Befragten ( $\mathrm{N}=368)$ suchten das Expertenforum auf, um allgemeine Informationen zum Thema „unerfüllter Kinderwunsch“ zu erhalten. Viele Personen wünschten zusätzliche Informationen über ihre derzeitige Behandlung und hatten Fragen zu Behandlungsoptionen der Kinderlosigkeit. Weitere Gründe waren Fragen zu den Ursachen der Kinderlosigkeit und zur Auswertung sowie Beurteilung von Untersuchungsergebnissen (Tabelle 3). Einige Frauen ( $N=39)$ konkretisierten ihre Gründe für die Konsultation des Expertenforums in den dafür vorgesehenen freien Textfeldern bzw. nannten dort andere Gründe für den Besuch, z. B. Fragen zur Fehlgeburt, Suche nach einer zweiten Meinung, Suche nach „Gleichgesinnten, die das Gleiche durchmachen wie ich“. 290 der Befragten gaben mehrere Gründe für ihren Besuch im Expertenforum an.

Tabelle 3: Themen und Anlässe der Anfragen an das Expertenforum

\begin{tabular}{lcc}
\hline & $\mathrm{N}$ & $\%$ \\
\hline Allgemeine Informationen & 368 & 72,9 \\
Fragen zur derzeitigen Behandlung & 228 & 45,1 \\
Fragen zu Behandlungsmethoden & 162 & 32,1 \\
Fragen zu den Ursachen der Kinderlosigkeit & 128 & 25,5 \\
Fragen zu Untersuchungsergebnissen & 111 & 22,0 \\
Sonstige & 39 & 7,7 \\
\hline
\end{tabular}

Insgesamt antworteten N=505; Missings: 18; Mehrfachnennungen möglich

\subsubsection{Bewertung des Expertenforums}

Viele Befragte hatten das Expertenforum schon häufiger besucht und schon früher eine oder mehrere Fragen gestellt („Mehrfach-Fragesteller“). Sie konnten daher auch die Qualität des Forums bewerten. Von den 225 Mehrfach-Fragestellern waren 123 (56,4\%; Missings: 29) mit den Antworten der Experten „zufrieden“; sieben waren „nicht zufrieden“; 88 Personen $(40,4 \%)$ antworteten mit „teils/teils“. In freien Textfel- 
dern konnten die Befragten das Expertenforum ausführlich beurteilen; 65 Befragte machten von dieser Möglichkeit Gebrauch - in der Mehrzahl ( $N=31)$, um Unzufriedenheit zum Ausdruck zu bringen. Hauptkritikpunkte waren die langen Beantwortungszeiten $(\mathrm{N}=12)$ und unbeantwortet gebliebene Fragen $(\mathrm{N}=13)$. Drei Frauen waren die Antworten der Experten unverständlich, während neun Frauen die Antworten als oberflächlich und fünf als unangebracht empfanden. Soweit die Anfragen an das Expertenforum selbst auch (in)direkte Aussagen zur Zufriedenheit oder zur Qualität enthalten, wird darauf in Kapitel 5.3.4 eingegangen.

\subsubsection{Expertenforum und Arztbesuch}

Die Hälfte der Mehrfach-Fragesteller (105/223) diskutierte die Antworten der Experten mit ihrem Arzt. Ein Viertel (51/221) wechselte aufgrund der Beratung im Expertenforum den Arzt bzw. suchte einen Spezialisten auf. 56 Personen $(25,2 \%)$ begannen auf Rat der Experten eine Therapie. Der Großteil der Besucher des Forums wechselte weder den Arzt (170 Befragte) noch begann er eine Therapie ( $N=166)$.

\subsubsection{Dauer und Behandlung der Kinderlosigkeit}

Die Paare, die zur Zeit der Erhebung das Expertenforum um Rat fragten, hatten im Mittel seit 28 Monaten einen (unerfüllten) Kinderwunsch (SD 29,8; Median 18; Range 0-240 ${ }^{1}$ ). Ihr erster Ansprechpartner für dieses Problem war der Frauenarzt (361 von 484 Betroffenen). Zweithäufigster Anlaufpunkt war das Expertenforum, gefolgt von Gesprächen mit Bekannten (Tabelle 4). Die Mehrzahl der Kinderwunschpaare (319/494) befand sich während der Befragung in Behandlung; im Durchschnitt seit 18 Monaten (SD 26,5; Median 10; Range 0,5-239,5). Hauptsächliche Behandlungsformen waren Hormonsubstitution ( $N=146)$ und künstliche Befruchtung ( $N=144)$. Einige Befragte $(\mathrm{N}=27)$ gaben alternative Behandlungsmethoden an. 54 Frauen berichteten in freien Textfeldern über zusätzliche Therapien und deren genaue Behandlungsphasen („Zur Zeit Hormonpause“, „Warten auf Befunde“) oder über anstehende Therapien. Fünf Frauen beschrieben alternative Heilmethoden bzw. Naturheilverfahren („Mönchspfeffer“, „Fußreflexzonenmassage“); 111 Frauen gaben mehrere Behand-

\footnotetext{
${ }^{1}$ Der Wert von max. 240 Monaten scheint sehr hoch; es gab aber mehrere Teilnehmer, die schon über 10 oder 15 Jahre einen Kinderwunsch hatten.
} 
lungen an. Insgesamt erhielten knapp 20\% (62/313) der Befragten psychologische Betreuung während ihrer Sterilitätstherapie.

Tabelle 4: Die Behandlungssituation des kinderlosen Paares

\begin{tabular}{lrr}
\hline & $\mathrm{N}$ & $\%$ \\
\hline Erster Ansprechpartner $^{1}$ & & \\
- Frauenarzt & 361 & 74,6 \\
- Expertenforum & 72 & 14,9 \\
- Bekannte & 26 & 5,4 \\
- Urologe & 9 & 1,9 \\
- Hausarzt & 8 & 1,6 \\
- Heilpraktiker & 4 & 0,8 \\
- Psychotherapeut & 2 & 0,4 \\
- Geistlicher & 2 & 0,4 \\
Derzeit oder früher in Behandlung & \\
- Hormontherapie & & 45,8 \\
- Künstliche Befruchtung (IVF, ICSI, IUI) & & 45,1 \\
- Beratung & 146 & 17,2 \\
- Operation & 144 & 8,8 \\
- Alternative Behandlungsmethoden & 55 & 8,5 \\
- Psychotherapie & 28 & 1,6 \\
- Sonstige & 27 & 16,9 \\
Psychologische Betreuung & 5 & \\
- Ja & 54 & 19,8 \\
- Nein & & 80,2 \\
\hline
\end{tabular}

\footnotetext{
${ }^{1}$ Prozentwerte jeweils auf der Basis von $\mathrm{N}=484$; Missings: 39

${ }^{2}$ Prozentwerte jeweils auf der Basis von $\mathrm{N}=320$; Missings: 28

${ }^{3}$ Prozentwerte jeweils auf der Basis von $\mathrm{N}=313$; Missings: 6
}

\subsubsection{Bewertung der ärztlichen Betreuung und Information}

Die Zufriedenheit mit der ärztlichen Betreuung war - bezogen auf das Problem der Kinderlosigkeit - insgesamt sehr hoch, insbesondere mit Ärzten in reproduktionsmedizinischen Zentren (Abbildung 4).

Zur Zeit der Befragung wurden 288 Probanden hauptsächlich von ihrem Frauenarzt und 165 in reproduktionsmedizinischen Einrichtungen betreut. Jeweils ca. drei Viertel der Befragten aus beiden Gruppen waren mit der Situation allgemein zufrieden. 


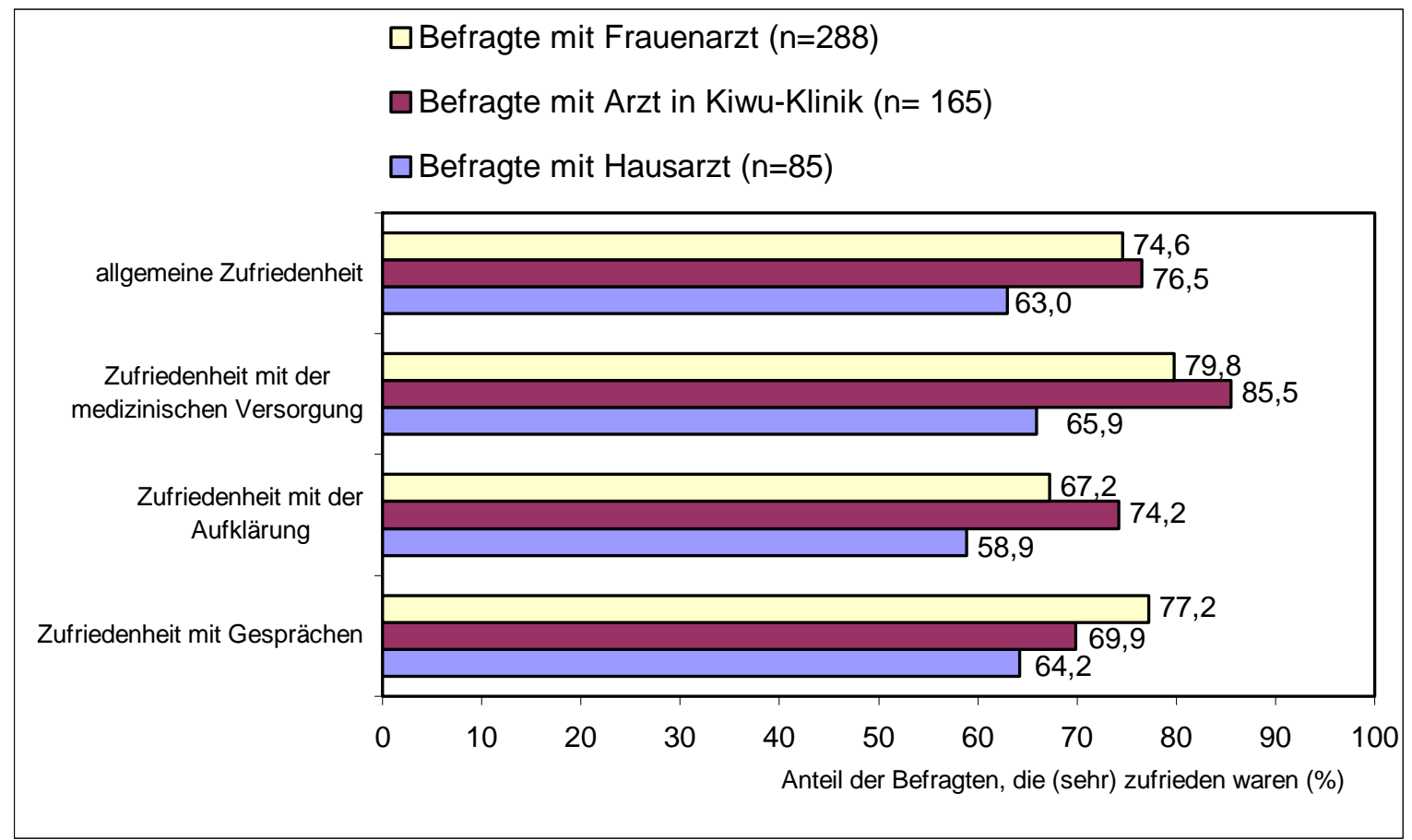

\section{Abbildung 4: Beurteilung der ärztlichen Betreuung}

Patienten, die wegen ihrer Kinderlosigkeit von ihrem Hausarzt betreut oder mitbetreut wurden $(\mathrm{N}=85)$, waren etwas seltener zufrieden $(63,0 \%)$. Besonders viele Patienten in spezialisierten Kinderwunschzentren (85,5\%) waren (sehr) zufrieden mit der medizinischen Versorgung. Etwas geringer war der Anteil zufriedener Patienten, wenn sie vom Frauenarzt $(79,8 \%)$ oder Hausarzt $(65,9 \%)$ betreut wurden.

Die Zufriedenheit mit dem Informations- und Aufklärungsangebot war im Durchschnitt geringer. So waren nur $74,2 \%$ der Befragten in reproduktionsmedizinischer Betreuung und nur noch 58,9\% der Befragten in hausärztlicher Betreuung mit der Aufklärung ihres Arztes zufrieden.

Etwas häufiger waren diejenigen Befragten mit der jeweiligen ärztlichen Betreuung zufrieden, die die Antworten des Kinderwunsch-Expertenforums mit ihrem Arzt besprachen bzw. besprechen können (80,1\%; Abbildung 5).

Drei Viertel der Befragten (220/285) konnten in Gesprächen mit ihrem Frauenarzt alle Probleme ansprechen, weniger Befragte waren es beim Hausarzt $(64,2 \%)$ und in reproduktionsmedizinischen Einrichtungen (69,9\%). 
143 der Befragten klagten darüber, dass verschiedene Probleme nicht besprochen werden konnten: am häufigsten psychologische Probleme $(N=79)$ gefolgt von sexuellen Problemen ( $\mathrm{N}=37)$, körperlichen Beschwerden und Problemen in der Partnerschaft (je $\mathrm{N}=30$ ).

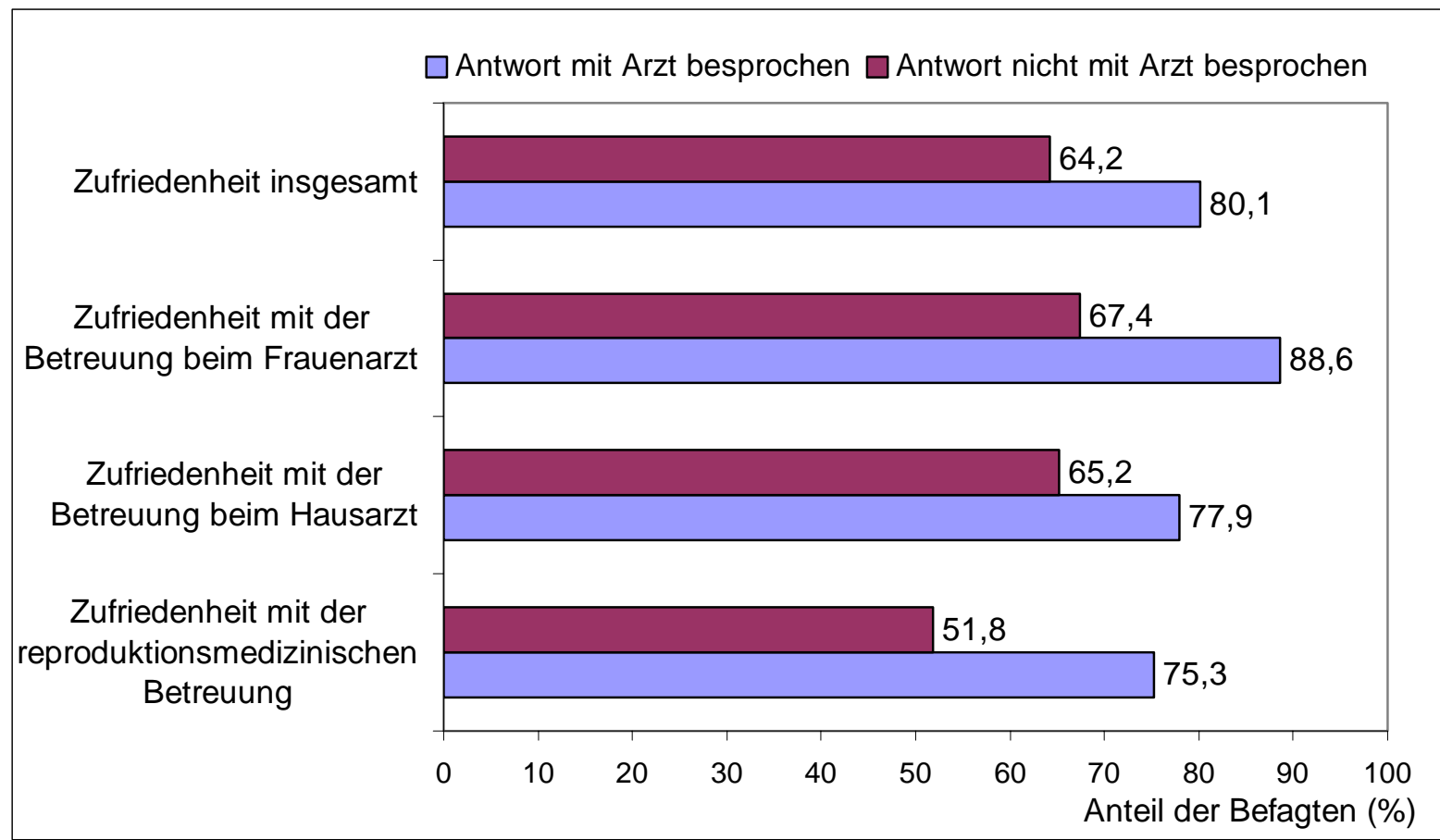

Abbildung 5: Zufriedenheit mit der ärztlichen Versorgung in Abhängigkeit davon, ob die Antworten der Experten mit den behandelnden Ärzten besprochen wurden

In freien Textfeldern äußerten 78 Personen Wünsche und Kritik. Diese bezogen sich auf die behandelnden Ärzte bzw. an die Reproduktionsmedizin allgemein: Hauptkritikpunkt war die fehlende Zeit $(\mathrm{N}=28)$ bei Beratungsgesprächen und Behandlungsterminen („Gefühl der Hektik“, „Fließbandabfertigung“). Genauso viele Frauen klagten über mangelnde Information und Aufklärung: Die Gespräche mit den Ärzten wären nicht ausführlich genug; es fehlten wichtige Informationen, wie z. B. Behandlungsalternativen, Risiken und Chancen der Therapie. Häufig würden die Details unverständlich erklärt. 20 Frauen vermissten bei den behandelnden Ärzten und dem Pflegepersonal das nötige Einfühlungsvermögen (,... möchte nicht nur auf meinen Zyklustag [1. Frage bei der Anmeldung] und meinen Unterleib reduziert werden.“, „,.. nicht auf dem Flur sagen, dass keine Schwangerschaft vorliegt"). Sie fühlten sich in ihren Problemen nicht ernst genommen („... das existenzielle Problem Kinderwunsch ist bei den Ärzten Routineangelegenheit"); darüber hinaus wünschten sie sich mehr 
Individualität („... ich fühle mich wie eine Legehenne“). Vielen Frauen war der (ehrlich gemeinte) Zuspruch durch die Ärzte, Ermutigung und emotionale Unterstützung ebenfalls sehr wichtig.

\subsection{Inhaltsanalytische Auswertung der Anfragen}

Die inhaltsanalytische Auswertung der Anfragen an das Expertenforum beruhte auf drei Datenquellen:

1. Anfragen an das Expertenforum, gekoppelt mit den Antworten auf unsere Internet-Befragung;

2. Zufallstichprobe früherer Anfragen an das Expertenforum;

3. Vergleich besonders kritischer bzw. typischer Anfragen an das Expertenforum mit den dazugehörigen Antworten der Internet-Befragung (Einzelfallanalysen; als separates Kapitel 5.3.6)

\subsubsection{Stichprobenumfang}

Von den insgesamt 3.840 im Zeitraum zwischen dem 28.07.1999 und dem 17.02.2001 gesammelten Fragen stammen 3.771 (98,2\%) von Frauen und 69 (1,8\%) von Männern. Der Fragenpool der Männer wurde komplett ausgewertet. Eine Stichprobe der Fragen von Frauen $(\mathrm{N}=245)$ wurde mittels Zufallszahlen gezogen. In die qualitative Auswertung gingen außerdem die an die Fragebögen gekoppelten Anfragen an das Expertenforum ( $\mathrm{N}=513)$ ein. Insgesamt wurden 827 Anfragen qualitativ analysiert. Aus technischen Gründen waren zehn an Fragebögen gekoppelte Anfragen nicht auswertbar.

\subsubsection{Funktion des Expertenforums}

Die inhaltsanalytische Auswertung sollte zeigen, mit welchen Fragen, Wünschen und Erwartungen Besucher an das Expertenforum herantreten.

Es lassen sich mehrere Funktionen erkennen, die dem Expertenforum aufgrund des konkreten Hilfebedarfs der Fragesteller zukamen. Als Hinweis auf die (potentielle) 
Funktion des Expertenforums wurden Äußerungen gewertet, in denen die Fragesteller den Wunsch nach Hilfe erkennen ließen und dieser Wunsch in der ambulanten Praxis oder im Krankenhaus bzw. in der reproduktionsmedizinischen Einrichtung offensichtlich nicht realisiert worden war und nun an das Expertenforum herangetragen wurde. Am häufigsten zeigte sich die Hilfefunktion des Expertenforums dort, wo jemand das Gespräch mit seinem behandelnden Arzt wünschte, es jedoch an Gelegenheit und Zeit fehlte (,....Mein FA [Frauenarzt] hat es immer eilig."). Bei einigen Fragestellern stand das Interesse an Informationen über Behandlungsmöglichkeiten und -alternativen im Vordergrund. Anderen fehlte für eine Aussprache das Vertrauen zum derzeit behandelnden Arzt. Einige Befragte hofften, dass das Expertenforum innen bei Unsicherheiten und Ängsten in Bezug auf die Infertilitätsbehandlung oder gegenüber fremden Ärzten beistehen könnte.

Tabelle 5 (S. 44) gibt einen schematischen Überblick - in Form von Hauptkategorien. 
Tabelle 5: Überblick über die Anfragethemen - in Form von Hauptkategorien

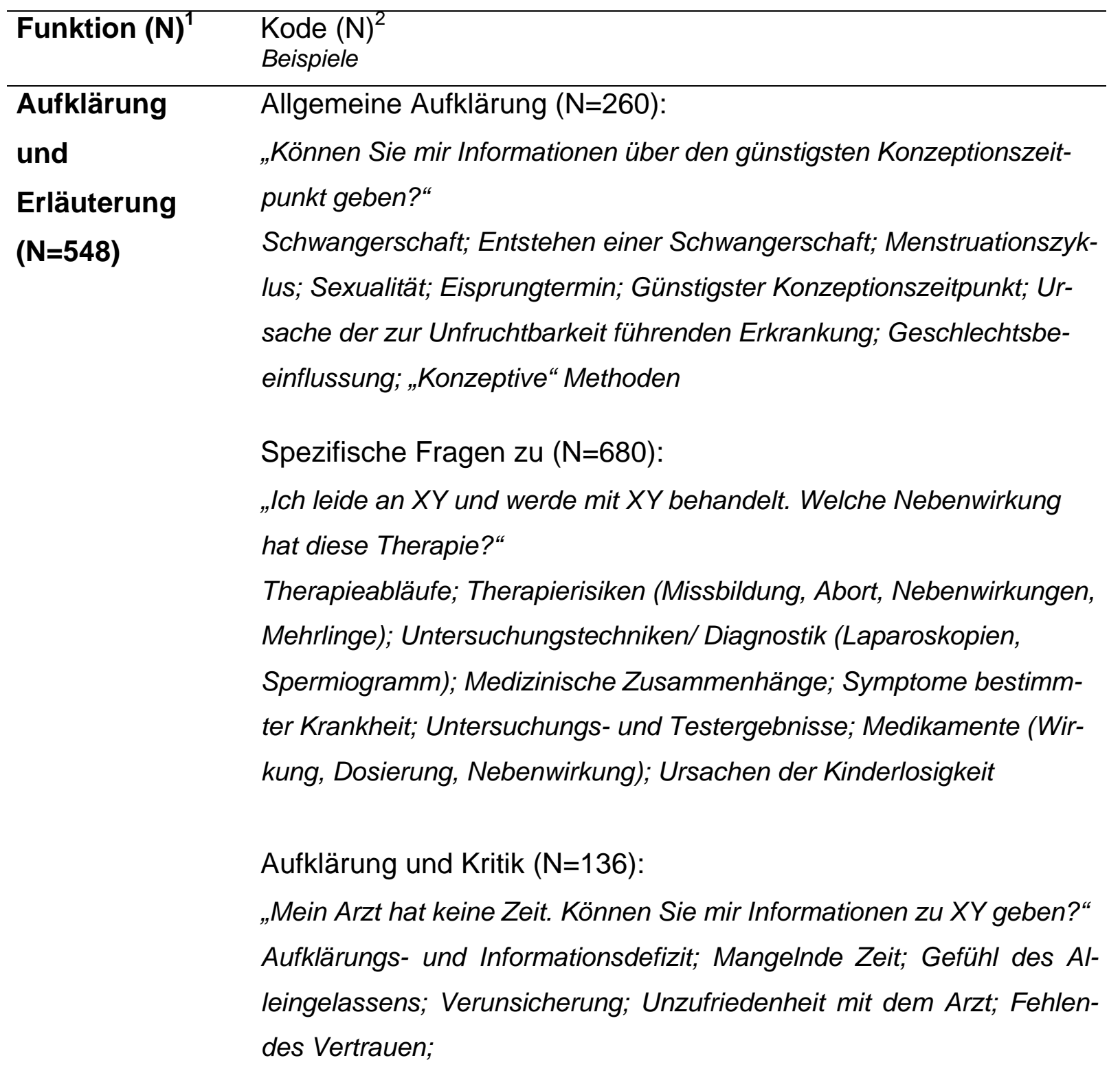
Unabhängige Zweite Meinung ( $N=374)$ :
medizinische „Mein Arzt behandelt mich mit XY. Würden Sie die Krankheit auch so Beratung behandeln?"
(N=396) Therapien (Konzeptionschancen, Risiken) Untersuchungsergebnisse;
Ursachen der Kinderlosigkeit;

Behandlungsmöglichkeiten ( $\mathrm{N}=172)$ :

„Welche (neuen) Therapiemöglichkeiten gibt es?"

Alternative Therapien (Homöopathika, Fußreflexzonenmassage)

Ernährung $(\mathrm{N}=7)$

\footnotetext{
${ }^{1} \mathrm{~N}$ entspricht der Anzahl der Dokumente, in denen die Hauptkategorien vorkommen.

${ }^{2} \mathrm{~N}$ entspricht der Anzahl der vergebenen Kodes.
} 
Tabelle 5 - Fortsetzung

Rechtsberatung $(\mathrm{N}=7)$

Konzeptionschancen ( $\mathrm{N}=10)$

Therapiekosten und Übernahme durch die Krankenkassen

$(\mathrm{N}=28)$

Möglichkeiten der Diagnostik ( $N=45)$

Lotse ( $\mathrm{N}=63) \quad$ Empfehlung, zum Arzt zu gehen $(\mathrm{N}=21)$

Empfehlung, in Kinderwunsch-Praxis zu wechseln ( $N=8)$

Arztwechsel $(\mathrm{N}=11)$

Empfehlung von geeigneten Fachärzten ( $\mathrm{N}=16)$

Empfehlung geeigneter Kliniken ( $\mathrm{N}=15)$ :

Tipps zur Informationssuche $(\mathrm{N}=3)$

\section{Emotionale Unterstützung $(\mathrm{N}=136)$}

Gefühlsäußerungen ( $\mathrm{N}=137)$ :

Angst; Unsicherheit; Panik; Frustration; Schuldgefühle; Trauer

Suche nach neuer Hoffnung $(\mathrm{N}=13)$ :

„Wieder Mut machen“;

Suche nach Gleichgesinnten ( $\mathrm{N}=9)$

Fehlende Unterstützung vom Partner $(\mathrm{N}=2)$

Neben diesen, in Tabelle 5 genannten Hauptkategorien, gab es verschiedene Anfragen, die weder eine spezielle Frage noch einen speziellen Wunsch enthielten $(\mathrm{N}=20)$, wie z. B. Diskussionen zwischen Forenmitgliedern, Danksagungen oder allgemeine Äußerungen.

Die Hauptkategorien wurden von den „Kode-Familien“ (s. Methodenteil) gebildet und werden im Folgenden näher beschrieben.

\subsubsection{Aufklärung}

Die Funktion „Aufklärung und Erläutern“ war in vielfältigen Wünschen nach Aufklärung und Informationen erkennbar ( $\mathrm{N}=548)$. Als wichtigste Hauptkategorie der Funktion „Aufklärung und Erläutern“ fanden wir die „allgemeine Aufklärung“ (N=260) z. B. über Vorgänge im Körper einer Frau im Zusammenhang mit der Sexualität und Fra- 
gen zum Menstruationszyklus. In diese Kategorie ließen sich ebenfalls Fragen zum „Entstehen einer Schwangerschaft“ und zur „Schwangerschaft" selbst sowie „Eisprungtermin“ bzw. „günstigster Konzeptionszeitpunkt“ zuordnen. Beispiele:

- $\quad$ Aufklärung über Sexualität

In dem Ovulationskalender hab ich die fruchtbaren Tage bestimmen lassen, aber was heißt das nun ? Wenn die z.B. von So bis Do sind, soll man dann ab So bis Do jeden Tag GV haben oder lieber nur jeden 2. Tag, oder wie ist das gemeint um möglichst schnell schwanger zu werden ? Sorry, aber da hab ich völlig Unterschiedliches gehört. (P110; FB 382.txt) $)^{1}$

- $\quad$ Aufklärung über Menstruationszyklus

Mein Zyklus beträgt ca. 26 Tage. Wann sind die fruchtbaren Tage? Wenn eine Regelblutung erfolgt, heißt das auch, dass ein Eisprung stattgefunden hat? Wird bei der Berechnung immer vom ersten Tag der Regelblutung gerechnet? (P63; FB 288.txt)

- $\quad$ Aufklärung über Schwangerschaft

Ich hatte laut O.[Ovulations-]Kalender am 29.09. die Einnistung (wenn überhaupt); ich hatte am 28. leichte Blutung beim Sex, und dachte mir noch nichts und dann hatte ich am 30. leichte Blutung. Ich hatte mal gelesen, dass es bei manchen Frauen bei der Einnistung leicht Blutungen auftreten. Meine Frage: stimmt das???? (P75; FB 309.txt)

Am häufigsten wurden die Ärzte des Expertenforums gebeten, Therapien $(\mathrm{N}=186)$ und Medikamentenwirkungen ( $N=76)$ zu erklären. Diese Anfragen wurden unter der Hauptkategorie „Erläuterung spezieller Fragen“ ( $\mathrm{N}=680)$ zusammengefasst. Die Studienteilnehmer wollten genaue Informationen über Therapieabläufe und -risiken (z. B. Missbildungs- und Abortrisiko) und den erwarteten Nutzen einer Behandlung (z. B. Konzeptionschancen). Weitere bedeutende Subkategorien waren das „Erklären von Befunden“ ( $\mathrm{N}=95)$, „Untersuchungstechniken“ ( $\mathrm{N}=27)$, wie z. B. Laparoskopien, und Informationen zur Diagnostik ( $=10)$, z. B. „Hormonuntersuchung“ oder „Bluttest“. Besucher des Expertenforums baten z. B. Spermiogramme, hinsichtlich der Verwirklichung des Kinderwunsches auszuwerten und zu erklären.

\footnotetext{
${ }^{1}$ Die Abkürzung P110 bedeutet, dass sich der Text in Atlas.ti im primary document 110 befindet; FB 372.txt ist die Nummer des dazugehörigen Fragebogens. Quellen mit P-Nummer und ohne FBNummer wurden aus dem Pool der Zufallsstichproben gezogen. Anfragetexte von Männern wurden zusätzlich mit einem $\mathbf{X}$ gekennzeichnet.
} 
- Bitte um Erklären von Therapieabläufen

Ich will im nächsten Zyklus meine noch eingefrorenen Eizellen einsetzen lassen. In den ersten drei Tagen soll ich mich in der Praxis melden. Gibt es einen Vorzyklus wie bei der IVF mit Pilleneinnahme oder wird beim nächsten "Eisprungstermin" eingesetzt. Ist das immer ca. am 14 Zyklustag oder kann man das etwas flexibel handeln. [...] Ich bin froh, wenn Sie mir etwas über den Ablauf (Termine, Medikamenteneinnahme etc. ) mitteilen könnten. (P94; FB 36.txt)

Ich stehe vor dem 1. IVF-Versuch, habe am 22. Zyklustag mit der Downregulation (tgl. 0,1 mg Uno-Enantone) begonnen und soll mich mit Beginn der Periode wieder in der KIWU-Praxis melden. Nun ist schon der 37 Zyklustag, ohne das sich etwas tut, SST war heute negativ. Kann es sein, dass die Periode völlig ausbleibt, und wenn ja, wann beginnt man dann mit der Stimulation? Vielen Dank im voraus für Ihre Antwort! (P39; FB 212.txt)

Einige Fragen zur derzeitigen oder angestrebten Behandlung spiegelten Ängste und Unsicherheit wider und waren nicht selten mit dem Wunsch nach mehr Information und Aufklärung seitens des behandelnden Arztes verknüpft. Einige Fragesteller berichteten in diesem Zusammenhang konkret von Defiziten in der Aufklärung durch ihren Arzt. Diese Anfragen wurden mit dem Kode der Hauptkategorie „Aufklärung mit

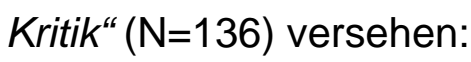

- Therapierisiken und -chancen

Vergangenen Mittwoch hatte ich um 11.00 Uhr eine Insemination bei meinem Gyn. Ab 4 Uhr bekam ich dann starke Bauchschmerzen und hohes Fieber. Um 8.00 Uhr wies mich mein Hausarzt ins Krankenhaus ein. Im Blut wurden erhöhte Werte festgestell, die auf eine Infektion hinwiesen. Eine Therapie mit Antibiotika wurde eingeleitet.(...) Eine Frage, wie groß ist nun die Chance überhaupt schwanger geworden zu sein, und wenn, schaden die ganzen Medikamente dem Kind? (...) Leider war ich mit der Aufklärung im Krankenhaus überhaupt nicht zufrieden und würde mich deshalb auf eine Antwort von ihnen sehr freuen. (P153; 235.txt)

- Erklären von Untersuchungsbefunden Nach einer neuerlichen Hormonuntersuchung teilte mein FA mir nun mit, dass meine Progesteronwerte katastrophal wären. Normal seien ein Wert zwischen 1000-2800 (???) und ich hätte 47. Somit wäre eine SS ausgeschlossen. Leider wurden mir die Werte nicht erklärt und auch keine Therapie vorgeschlagen. Können Sie mich aufklä- 
ren? Habe ich ggf. keinen Eisprung und sind meine Werte wirklich so katastrophal? Diesen FA suche ich natürlich nicht nochmals auf. Raten Sie mir ggf. zu einer KiwuSprechstunde oder kann ich selbst etwas tun? (P196; FB 565.txt)

In vielen Anfragen wurde die mangelnde Zeit während des Arztgesprächs kritisiert. Diese Frauen äußerten Gefühle des „Alleingelassenseins“ und der Verunsicherung. Einige Frauen beschrieben ihre Angst vor bestimmten Untersuchungen mit der Bitte, diese zu erklären. Sie schienen von ihrem Arzt nicht ausreichend aufgeklärt zu sein oder hatten beängstigende Informationen aus anderen Quellen bezogen:

- Erklären von Untersuchungstechniken:

Meine Ärztin möchte im nächsten Zyklus (z.Zt. ist Pillenpause wg. Weihnachten) wieder mit Fertinorm stimulieren und gleichzeitig eine röntgenologische Eileiterüberprüfung (HSG) machen lassen. Ich habe einen Horror davor, weil das so schmerzhaft sein soll. Jetzt lese ich hier im Forum öfter etwas von Ultraschall +Kontrastmittel zur Überprüfung der Eileiter. Ist das vielleicht sinnvoller? Einen Follikel zu stimulieren und dann mit Röntgenstrahlen zu beschießen ist wahrscheinlich nicht so ideal. Können Sie mir vielleicht weiterhelfen? (P148; 230.txt)

Zur Kode-Familie „Aufklärung und Erläutern“ gehörten zusätzlich die Aufklärung über "Symptome“ ( $N=45)$, „medizinische Zusammenhänge“ ( $N=21)$ und „Ursachen für Fehlgeburten bzw. Abortrisiken“ ( $N=30)$.

Die Kategorie „Aufklärung und Erläutern“ ist schwer von der Kategorie „medizinischer Berater“ bzw. der Subkategorie „zweite Meinung“ zu trennen. Es gibt viele Schnittpunkt zwischen diesen beiden Kode-Familien: Z. B wurden die Kodes „Auswerten von Befunden“ und "Einschätzen von Schwangerschaftschancen“ teils in die Subkategorie „zweite Meinung“, teils in die Kategorie „Aufklärung und Erläutern“ eingeordnet.

\subsubsection{Unabhängige medizinische Beratung}

Anders als in der Hauptkategorie „Aufklärung mit Kritik“ wurden hier diejenigen Fragen zugeordnet, in denen ausschließlich um eine zweite Meinung gebeten wurde, ohne dabei die Meinung der bisher behandelnden Ärzte negativ zu schildern. 
Viele Besucher des Expertenforums sahen im Ärzteteam einen medizinischen Berater, der über Behandlungsmöglichkeiten informiert und bei der Suche nach behandelnden Ärzten hilft. Einige Fragende hatten es sogar vorgezogen, statt ihres behandelnden Arztes einen Arzt im Expertenforum um Aufklärung über die Infertilitätsbehandlung und ihre Risiken zu bitten. Die Ärzte des Forums fungierten sowohl als Ergänzung, als auch als Alternative zum behandelnden Spezialisten.

Sehr häufig wurden die Ärzte des Expertenforums als „Zweitgutachter“ eingesetzt: In 374 Anfragen wurden sie um eine zweite Meinung hinsichtlich der Therapie, Erfolgschancen und der ärztlichen Tätigkeit gebeten. Die Mehrzahl der Fragesteller ( $N=117)$ hatte in dieser Kategorie Fragen zu ihrer derzeitigen oder geplanten Therapie. An die Experten wurde also die Aufgabe (die Erwartung) herangetragen, die Therapiemethode des behandelnden Arztes zu beurteilen. In vielen Anfragen sollte die derzeitige oder geplante Behandlungsmethode hinsichtlich der Konzeptionschancen objektiv eingeschätzt werden ( $N=63)$. Beispiel:

- Bewertung der Therapie u.a. hinsichtlich Konzeptionschancen

Ich beginne am Montag mit der 2. Stimulationsphase für IVF und ICSI. Mein KIWUArzt hat mir nun zwecks Embryonentransfer zur Mini-Tet geraten.(..) Zudem rät er uns, ein sog. Assisted Hatching durchführen zu lassen.(..) Was halten Sie von dieser Methode? Angeblich soll sie nochmals die Schwangerschaftsrate erhöhen? Ich bin unsicher und habe natürlich auch Angst, dass dann, falls es zur Einnistung käme, ein behindertes Kind die Konsequenz wäre. Arbeiten Sie in T. auch mit solchen Methoden? (P24; 12.txt)

Wie schon erwähnt, wurde unter der Funktion „Beratung“ und Hauptkategorien „zweite Meinung“ auch die „Auswertung von Befunden“ subsumiert, denn viele Besucher des Expertenforums wollten ihre Untersuchungsergebnisse von unabhängigen Sachverständigen noch einmal „gegengecheckt“ bzw. interpretiert haben.

Ein anderer Schwerpunkt waren Anfragen zu verschiedenen Behandlungsmöglichkeiten ( $\mathrm{N}=172)$, wie z. B. nach Naturheilverfahren. Des Weiteren wurden Fragen nach effektiven Diagnosemöglichkeiten für die jeweiligen Fälle gestellt $(\mathrm{N}=45)$. Das Expertenforum wurde ebenfalls genutzt, um sich über die verschiedenen Therapierisiken und -chancen verschiedener Therapien, Arzneimittelneben- und Wechselwirkungen beraten zu lassen - als Entscheidungshilfe für oder gegen eine Therapie. 
- Entscheidungshilfe:

Nach 2 Fehlgeburten nahm ich für einige Zyklen Duphaston in der 2. Zyklusphase. Nachdem ich aber in letzter Zeit unter Schwindelgefühlen litt, habe ich mich entschlossen, es nicht mehr zu nehmen. Ich würde gerne wissen für wie wichtig sie die Einnahme des Medikaments erachten. Für meine Fehlgeburten wurden keine Ursachen festgestellt. Duphaston wurde mir (meinem Eindruck nach) unter dem Prinzip "hilft's nix, schad's nix" verordnet. (P46; 14.txt)

Nächste Wochen starten wir unseren 3. ICSI-Versuch. Ich habe bislang immer ab dem Stimulationsbeginn ASS 100 genommen. Jetzt sagt mein Arzt, ich soll das diesmal nicht nehmen, weil er sowieso nicht an die Wirkung glaubt und ich schon genug Medikamente bekommen würde. Ich habe solche Angst, etwas falsch zu machen. Nachdem, was mein Arzt sagt, tendiere ich jetzt eher dazu, es nicht zu nehmen, aber er überlässt die Entscheidung allein mir. Was machen Sie, verschreiben Sie es Ihren Patientinnen? (P78, 168.txt)

Zusätzlich wurde um Ernährungsberatung, Rechtsberatung und um Ratschläge für Arztgespräche gebeten. Einige Frauen suchten Informationen zu Therapiekosten und deren Übernahme durch die Krankenkassen.

Vor allem Frauen, denen gerade erst Schwierigkeiten bei der Verwirklichung ihres Kinderwunsches bewusst geworden war $^{1}(\mathrm{~N}=67)$, ließen sich über verschiedene „konzeptive" Methoden, wie Erstellen eines Ovulationskalenders oder Handhabung eines Persona-Tests sowie über Gründe für die mögliche Infertilität $(\mathrm{N}=20)$ beraten.

Ein anderes häufiges Anfragethema dieser Frauen war die Schwangerschaft und Zyklusnormalisierung nach langer Pilleneinnahme $(\mathrm{N}=33)$.

- Ursachen der Kinderlosigkeit

"Hallo, ich weiß es klingt dumm aber mein Freund und ich wünschen uns seit langen 1 Kind. Da ich bis letztes Jahr Sommer aber noch in der Ausbildung war, habe ich erst danach angefangen die Pille abzusetzen. Nun ist das schon 7 Monate her und ich bin immer noch nicht schwanger. Meine Mens. ist auch sehr unregelmäßig geworden. (...) Beim FA war ich auch schon, sie meint es wäre alles in Ordnung. Liegt es vielleicht an meinem Freund? In letzter Zeit hab ich aber auch diese "Freßatta-

\footnotetext{
${ }^{1}$ Hierzu zählten wir Frauen, die seit weniger als einem Jahr versuchten, schwanger zu werden.
} 
cken", und die Übelkeit, aber der SST zeigt immer negativ an. Können sie mir vielleicht weiterhelfen. (P386; FB 1050.txt)

Seit 7 Monaten versuchen wir erfolglos schwanger zu werden. Mir kam der Verdacht, dass das mit der Pille zusammenhängen könnte, da ich wegen Thrombosegefahr nur eine ohne Östrogene nehmen durfte (hab leider den Namen vergessen). Hatte während der Einnahme keine Periode. Nach Absetzen bekam ich dann 1 Woche später die erste Mens und seit dem regelmäßig (28/29 Tage). Wie lange braucht der Körper um sich von dieser Hormonumstellung zu erholen? Der Zeitraum kommt mir doch etwas lang vor. (...) Was können Sie uns raten? (P372; FB 1011.txt)

\subsubsection{Lotsenfunktion}

Viele Frauen baten - neben der Einschätzung ihrer Situation - um eine Empfehlung seitens des Experten, an welche Fachärzte sie sich wenden sollten. Hauptsächlich wurde nach Namen von „Spezialisten“ oder Adressen von spezialisierten Krankenhäusern in der näheren Umgebung gefragt $(\mathrm{N}=31)$. Die Funktion des Expertenforums gleicht der eines "Lotsen“ ( $\mathrm{N}=63$ ), der die Frauen entweder an das Gesundheitssystem heranführt oder sie zu den verschiedenen Fachärzten delegiert. Anfragen wurden auch dann unter die Funktion "Lotse“ eingeordnet, wenn eine Stellungnahme zu einem beabsichtigten Wechsel in eine Kinderwunsch-Praxis erbeten oder nach dem geeigneten Facharzt (Frauenarzt, Urologe...) gefragt wurde:

- Heranführen an das Gesundheitssystem:

"Ich bin 24 Jahre alt und versuche seit 8 Monaten schwanger zu werden. Habe vorher 10 Jahre lang die Pille genommen. Im letzten Zyklus habe ich das erste Mal meine Temperatur gemessen - es war kein Eisprung zu erkennen. Mein Zyklus ist unregelmäßig (...). Würden Sie mir empfehlen, mich von meiner Frauenärztin einmal untersuchen zu lassen, oder sollte ich noch abwarten? Was würde sie als erstes untersuchen? Ich habe schon viel über Mönchspfeffer und Clomifen gelesen. Würde von diesen Medikamente etwas für mich in Frage kommen? Oder denken sie, dass sich mein Zyklus noch von alleine einspielt? (P378; FB 1027.txt)

- Empfehlung von Ärzten und Kliniken:

Wir wünschen uns seit ca. 3 Jahren ein Kind und sind seit April 2000 im Kinderwunschzentrum Dr. $K$ in $M$ in Behandlung. Nachdem feststand, dass die Ursache bei meinem Mann liegt und nur ICSI infrage kommt, haben wir nun im Jahr 20012 erfolg- 
lose Versuche hinter uns (...). Wir sind ziemlich verzweifelt. Können Sie uns einen Tipp bzw. eine Adresse in M od. Umgebung nennen, die auf Alternativmedizin (z. B. Homöopathie, Akupunktur o.ä.) in dem Thema spezialisiert sind? Ggf. könnte man den nächsten Versuch damit unterstützen? (P303; FB 898.txt)

- Delegieren an geeignete Fachärzte und spezialisierte Zentren:

"Hallo, Mein Freund hat gerade sein Ergebnis von seinem Spermiogramm erhalten.(...) Ich bin jetzt ziemlich geschockt, weil wenn ich in der Suchmaschine OATSyndrom eingebe, haben alle wenigsten 1 Millionen und mein Freund unter 50.000. Ist das überhaupt noch ein OAT-Syndrom? Oder ist es noch viel schlimmer? Was haben wir für Möglichkeiten, Kiwu-Praxis aufsuchen? (P483; FB 1259.txt)

\subsubsection{Emotionale Unterstützung}

In vielen Anfragen wurde deutlich, dass die Frauen - neben professioneller Auskunft und Informationen - auch emotionalen Beistand bei den Experten suchen $(\mathrm{N}=136)$. Gefühle, wie Angst, Unsicherheit und des Alleingelassenseins wurden in vielen Fragen deutlich. Die Anfragen waren stark emotional „besetzt“; manchmal wirkten sie fast panisch. Die Gefühle selbst wurden teils latent geäußert, teils wurden sie abrupt zwischen eine Flut von Fragen gepresst; Versalien symbolisierten in anderen Mails das Innenleben. Unsicherheit wurde am häufigsten mitgeteilt ( $N=49)$, meist im Zusammenhang mit Fragen zu Therapien und ihren Nebenwirkungen. Gerade bei Frauen mit einer langen Krankheits- und Behandlungsgeschichte fiel ein hoher Leidensdruck auf. In großer Hoffnung auf bessere Erfolgschancen wandten sie sich an die Ärzte des Expertenforums mit Fragen nach neuen Behandlungsmöglichkeiten. In dieser Situation wünschten viele Frauen eine Einschätzung ihrer Situation bezüglich des Kinderwunsches.

Ich schreibe Ihnen, weil meine Nerven blank liegen. Im Januar 2000 haben wir uns an die Kinderplanung gemacht. Bis Dezember 2000 habe ich es der Natur überlassen. 01/2001 FA: Testosteronspiegel sei zu hoch $(1,43)$, deshalb Dexamethasoneinnahme. 02/2001 Fehlgeburt (Windei) (7.SSW) und Ausschabung. 03/2001: Bauchspiegelung (Endometriosezysten am Eierstock) 08/2001 Clomifen und Utrogest: zahlreiche Follikel und Zysten. Durch Utrogest Verlängerung des Zyklus. Die FÄ können mir nicht helfen, da alles o.k. sei. Ich habe regelmäßige Eisprünge (Clomifen war nur ein Versuch) und das Spemiogramm meines Mannes ist auch o.k. Sie sagen ich soll es mit autogenem Training oder sowas versuchen. Ich kann und will so nicht mehr wei- 
terleben. Ich bin 24 (bald 25), wir sind bald 6 Jahre zusammen und wünschen uns bald 2 Jahre ein Kind. Was sollen wir noch tun??? Ich habe Angst vor einer KiWuKlinik, weil ich weiß, dass ich auch dort versagen werde... Bitte helfen Sie mir weiter"! (P131; FB 423.txt)

"Großer H I L FE R U F! Kurz zu meiner traurigen Geschichte: Ich in 29 Jahre und seit 09'2000 im Kiwu -Zentrum Wiesbaden. Zu meiner Endo[metriose] Geschichte: 1993=2 mal Bauchspiegelung 1994=2 mal Bauchspiegelung 1996=1 mal Bauchspiegelung und Bauchschnitt (Wechseljahrtherapie mit Winobanin 1/2 Jahr lang) 1997=1 mal Bauchspiegelung und Bauchschnitt, Entfernung vom linken Eierstock!(Wechseljahrtherapie mit Winobanin 1/2 Jahr lang) 2000= Bauchspieglung und Kur von 3 Wochen. Kiwu-Behandlung: 1. ICSI Punktion 09'2000: 14 Eizellen, alle befruchtet, 3 eingefroren, 2 zurück gesetzt bekommen, NEGATIV 2. ICSI Kryo 01'2001: 3 Eizellen, positiv, schwanger bis zur 5. Woche 3. ICSI Punktion 06'2001: 13 Eizellen, 9 befruchtet, 3 eingefroren, 3 Eizellen zurück gesetzt bekommen, NEGATIV 4. ICSI Kryo 10'2001: 3 Eizellen, alle 3 gehatched, NEGATIV 5. ICSI Punktion 02'2002: 7 Eizellen, 5 befruchtet, keine eingefroren!, 3 zurück gesetzt bekommen, NEGATIV Könnte es vielleicht doch mit meiner Endometriose zusammenhängen????? Ist vielleicht eine Wechseljahrtherapie oder eine Downregulation von 3 Monaten zu empfehlen? Oder ist eine Blutuntersuchung HLA zu empfehlen?? HILFE!!, ich weiß einfach nicht weiter, bin am Boden zerstört und völlig hilflos. Mir ginge es auch gleich viel besser, wenn ich wüsste, dass die $K K$ weitere Versuche zahlen. Ist es richtig, dass die KK weitere Versuche zahlen, wenn man mal ein positives Ergebnis hatte????, und wenn ja, wie viele Versuche übernimmt die KK, nochmals 4 Versuche???, oder weniger??? (P478; FB 1248.txt)

Einige Frauen litten ebenfalls sehr unter fehlender partnerschaftlicher Unterstützung, was die folgende Anfrage an das Forum möglicherweise stärker motiviert haben könnte als die medizinische Situation selbst:

Hallo! Ich habe eine Frage und hoffe, dass sie mir helfen können. Ich habe vor 1 Monat die Pille abgesetzt und hatte dann 2 Wochen später eine Blutung von einer Woche. Nun müßte ich heute eigentlich meine Tage bekommen. Ich habe allerdings seit einer Woche ab und zu ein Ziehen im Bauch und leichte Übelkeit und die Brüste tun mir weh beim Anfassen. Jetzt kommt noch hinzu das ich mir so sehr ein Kind wünsche. Ich habe schon gleich heute einen Schwangerschaftstest gemacht, aber der war natürlich negativ. Mein Freund sagt immer: Wenn ich mir so sehr ein Kind wünsche dann klappt es sowieso nicht. Das nimmt mir total meine Hoffnung. Er kann 
mich gar nicht verstehen wie ich mich fühle. Ich denke den ganzen Tag nur daran an ein Baby zu bekommen. (P67; FB 294.txt)

\subsubsection{Stichprobe der Männer}

Die Stichprobe der Männer umfasste 69 Anfragen aus der Zufallsstichprobe und vier Anfragen, die an einen Fragebogen gekoppelt waren.

Die Anfragen unterschieden sich in ihrer Intention nicht wesentlich von denen der Frauen, wie sie in Tabelle 3 und 5 zusammengefasst wurden. Ein Hauptthema der Anfragen war die Auswertung des jeweiligen Spermiogramms und dessen Beurteilung im Zusammenhang mit dem Kinderwunsch sowie die Erörterung positiver und negativer Einflussfaktoren auf das Spermiogramm $(\mathrm{N}=44)$. Wie bei den Frauen stellten auch die Männer häufig Fragen zur derzeitigen oder geplanten Therapie ( $N=15)$. Ebenfalls baten sie oft um eine zweite Meinung hinsichtlich Therapie, Erfolgschancen und Risiken. Von großem Interesse waren schließlich auch Informationen über bestimmte Behandlungsmöglichkeiten und -alternativen $(\mathrm{N}=12)$. Anders als bei den Frauen fanden sich in den Anfragen der Männer kaum emotional gefärbte Äußerungen, wie Angst, Hoffnungslosigkeit oder Trauer. Nur ein Mann äußerte sich unzufrieden und enttäuscht über die ärztliche Versorgung wegen ungenügender Aufklärung. Die Anfragen der Männer waren zumeist wesentlich kürzer und präziser formuliert. Zwei typische Beispiele:

'Hat es irgendeinen Sinn, bei einem Spermiogramm mit weniger als 1 Million Spermien/ml Padutin oder Zink einzunehmen??? Danke für die Antwort! (P254; X0008.txt)

In unserer Klinik ist es üblich vor ICSI generell eine Chromosomenanalyse beider Partner durchzuführen.

1. Ist das bei Ihnen auch üblich?

2. Was kann bei einer solchen Untersuchung genau festgestellt werden?

3. Wie lange muß man auf das Ergebnis warten?

Vielen Dank für Ihre Antworten!! [...] Holger (P255; X0009.txt) 


\subsubsection{Implizite Bewertung des Expertenforums}

In vielen begleitenden Kommentaren - parallel oder zusätzlich zu einer Anfrage - kam eine überwiegend positiv Bewertung des Expertenforums direkt oder indirekt zum Ausdruck; im Folgenden z. B. ein deutliches Lob für das Expertenforum bzw. die Arbeit der Ärzte:

...Ihre Arbeit und die, Ihrer Kollegen ist wirklich ein großer Verdienst, den man mit Worten alleine gar nicht genug würdigen kann. DANKE! (P87; 176.txt)

Viele Besucher bedankten sich dafür, dass ihnen die Experten neue Hoffnung bei langwierigen Therapien und Rückschlägen gegeben hatten.

...Vielen Dank für Ihre Antwort! Sie haben mir wieder Mut gemacht! (P120; 205.txt)

Nur selten wurde Kritik an der Arbeit des Expertenforums geäußert. Diese bezog dann sich vor allem auf lange Antwortzeiten, unbeantwortete Anfragen und unverständliche Antworten.

Schade, dass Sie meine Frage vom 07. 11. 2001 nicht beantwortet haben! Aber das bin ich ja schon gewohnt, hatte schon mal eine andere Frage und die hat mir auch niemand beantwortet!!! (P228; FB 633.txt)

\subsubsection{Bewertung der ärztlichen Betreuung}

In 136 Anfragen wurde die ärztliche Betreuung am Heimatort offen kritisiert: z.B. die mangelnde Aufklärung ( $N=29)$. Viele Frauen fühlten sich nicht umfassend durch ihren Arzt informiert; glaubten teilweise sogar, dass ihr Arzt Informationen zurückhalte. In einigen Anfragen führten die Frauen die fehlende Zeit und die daraus resultierende mangelnde Aufklärung auf die Überlastung der Ärzte zurück. So löst sich der scheinbare Widerspruch, dass diese Frauen mit der ärztlichen Betreuung trotzdem zufrieden waren. In anderen Fällen wurde die Arbeit des behandelnden Arztes auch gewürdigt. Als weiterer Kritikpunkt an der ärztlichen Tätigkeit wurden „Arztwechsel“ z.B. aufgrund von vermeintlich falscher Behandlungsmethoden oder „unverstanden Ängste und Sorgen" gewertet. Auch Patientinnen, die nicht ihren Arzt wechselten gaben an, sich oft in ihre Gedanken und Ängsten allein und unverstanden zu fühlen, oder 
sie berichteten, dass ihnen das Vertrauen fehlte, sich mit ihren Sorgen an den Arzt zuwenden.

- Aufklärungsdefizite

Seltsamerweise hat er mir die Herausgabe des OP-Berichtes meiner Abrasio die er auf Grund einer FG Ende der 6. SSW durchführte verweigert. Was soll denn das, es geht doch um mich - ich habe doch ein Recht zu erfahren was da im OP abgelaufen ist, oder liege ich da falsch? (...) Ich habe lediglich eine Kopie des Befundes des Einsendematerials bekommen (von d. Abrasio). Bitte übersetzen Sie mir dieses fachchinesisch, den ich bin fix und fertig weil ich das Gefühl habe er hatte mir etwas verschwiegen. (...) Bitte helfen sie mir, ich bin selber Arzthelferin aber auf fremden Fachgebiet, verstehe daher nur die Hälfte - aber das bisschen was ich verstehe beunruhigt mich, denn der Arzt sagte mir ich wäre kerngesund gewesen. (...) Dadurch dass er mir den OP-Bericht verweigert bin ich nur noch mehr verunsichert. (P105; FB 377.txt)

- $\quad$ Fehlende Zeit

Leider hat meine FA erst im Februar einen Termin frei (It. Sprechstundenhilfe ist es bei einem Kind [gemeint: sekundäre Sterilität] nicht dringend) (P315; FB 967.txt)

- Kein Vertrauen

Leider traue ich mich nie, meinen FA zu fragen, weil er immer sagt, ich soll mir keinen Stress machen, aber das ist einfacher gesagt als getan...(P336; FB 5.txt)

Kann es sein, dass ich schwanger bin? Ich habe Angst, dass meine FÄ mich für verrückt erklärt, wenn ich es nicht bin, deswegen wollte ich sie erst zu rate ziehen! (P245; FB 700.txt)

\subsubsection{Einzelfallanalysen}

Eine wichtige Säule der Datenauswertung war die Einzelfallanalyse. Nach Sichtung des Materials aus Pool der Anfragen an das Expertenforum werden im Folgendem typische Konstellationen, die natürlich im Einzelfall vielfältige Varianten und Abweichungen aufwiesen, illustriert. 


\subsubsection{Beispiel einer beginnenden Hilfesuche}

Ein Teil der Frauen wandte sich an das Expertenforum in folgender Situation: Nach 3 bis 6 Monaten ungeschützten Geschlechtsverkehr wurde ihnen das Ausbleiben der Schwangerschaft bewusst. Sie konsultierten dann als erstes ihren Frauenarzt, um organische Ursachen für die ausbleibende Schwangerschaft auszuschließen. Zeitgleich oder auf Anraten des Arztes begannen viele betroffenen Frauen mit natürlichen „konzeptiven“ Methoden, wie z. B. Eisprungmessung durch die Temperaturmethode oder LH-Sticks. So auch eine 31-jährige Frau, die seit 6 Monaten versucht hatte, schwanger zu werden. Sie hatte das Expertenforum durch Zufall im Internet entdeckt und stellte zum Zeitpunkt der Befragung zum ersten Mal eine Frage. Sie suchte im Expertenforum nach Rat, nachdem sie sich wegen ihres unerfüllten Kinderwunschs an ihren Frauenarzt gewandt hatte. Eine Behandlung wurde durch den Frauenarzt noch nicht begonnen:

Hallo, wir wünschen uns ein Baby, aber ich werde nicht schwanger auch nach 6 Monaten versuchen. Ein Kind haben wir schon. Aber es will nicht klappen obwohl ich schon fast ein Jahr LH-Sticks benutze und mein Eisprung ziemlich gut weiß. Was kann ich noch tun mein FA meint ich wäre gesund. Was soll ich nur tun??? Ich denke an nichts anderes mehr vielleicht ist das mein Fehler!!! Vielen Dank im Vorraus (P81; FB 323.txt)

\subsubsection{Beispiel eines langen Therapieweges}

Während Frauen am Beginn ihrer Kinderlosigkeit eher allgemeine Fragen zum unerfüllten Kinderwunsch hatten, stellten Frauen, die sich schon länger in reproduktionsmedizinischer Betreuung befanden, präzisere Fragen zu speziellen Therapieverfahren. Viele schilderten (meist in relativ langen Mails) verschiedene Untersuchungen und Therapieansätze, die sie während ihrer Kinderwunschbehandlung durchlaufen hatten. Eine Betroffene, 41 Jahre, seit 10 Jahren ungewollt kinderlos, entdeckte das Expertenforum in den Medien und stellte zur Zeit der Umfrage das erste Mal eine Frage an das Expertenforum. Der Frauenarzt war ihr erster Anlaufspunkt wegen ihres unerfüllten Kinderwunsches; dort befindet sie sich seit fünf Jahren in Behandlung. Die Patientin unterzog sich zum damaligen Zeitpunkt einer Hormonbehandlung mit Clomhexal und Utrogest. Die Betreuung durch ihren Frauenarzt und durch die Reproduktionsmediziner bewertete sie jeweils mit „gut“, die Aufklärung im reprodukti- 
onsmedizinischen Zentrum mit „sehr gut“. Die Befragte gab ebenfalls an, sich mit ihren behandelnden Ärzten im reproduktionsmedizinischen Zentrum über alle Probleme, die während der Therapie auftraten, unterhalten zu können. Mit ihrem Frauenarzt sei dies nicht immer möglich. Neben der Kinderwunsch-Therapie wird sie psychotherapeutisch betreut.

Sehr geehrte Frau Dr. B., wir haben ein Problem, vielleicht können Sie uns einen Rat geben. Es geht um Kinderwunsch, wir versuchen es schon sehr lange,1997 ist bei mir eine Eileiterdurchlässigkeit überprüft worden und es war alles in Ordnung, 1998 bin ich endlich Schwanger geworden das war im Mai 1998, im August 1998 in der 14.Schwangerschaftswoche verlor ich mein Baby (Bub), und seitdem klappt es nicht mehr ich versteh bloß nicht warum? Wir beide sind gesund. Nun möchte ich ein paar Zeilen zu uns schreiben: Wir sind nicht verheiratet. Mein Lebensgefährte ist 64 Jahre alt, aber laut Spermiengram ist alles in Ordnung. Ich selbst bin 41 Jahre alt und wie gesagt 1998 zum ersten Mal schwanger geworden, ich nehme Hormontabletten Clomhexal 50 und Utrogest. Habe dadurch jeden Monat meinen Eisprung. Meine Vermutung warum ich nicht Schwanger werde, dass ich ein bisschen vollschlank bin. Können Sie, Frau Dr. B., es bestätigen. Können wir eine künstliche Befruchtung machen lassen vielleicht mit fremd Samen ist das bei uns erlaubt?? Mein Lebensgefährte währe einverstanden. An wen sollen wir uns wenden. Es wäre sehr schön, wenn Sie uns vielleicht weiter helfen können. Im voraus vielen Dank Frau Dr.B.! (P52; FB 24.txt)

Die folgende Anfrage ist von einer Betroffenen, die ihre behandelnden Ärzte schlechter bewertet: Die 35-jährige, seit 2 Jahren ungewollt kinderlose Frau, hatte das Expertenforum durch Zufall im Internet entdeckt und schon mehrere Male eine Frage an die Experten gerichtet. Im Expertenforum hatte sie sich auch wegen ihrer Kinderlosigkeit zum ersten Mal Rat eingeholt. Sie gab in unserer Internet-Befragung an, mit der Arbeit der Experten bzw. mit deren Antworten zufrieden zu sein. Sie besprach die Antworten der Experten mit ihrem Arzt und wechselte aufgrund der Beratung der Experten zu einem spezialisierten Frauenarzt. Dort unterzog sie sich zum Zeitpunkt der Befragung seit 12 Monaten einer Hormontherapie. Die Betreuung durch ihren Frauenarzt bewertete sie als „sehr schlecht“, ebenfalls ist sie mit der Aufklärung durch den Frauenarzt „eher unzufrieden“. Des Weiteren gab sie an, mit ihrem Frauenarzt nicht über ihre Probleme sprechen zu können: Probleme über die sie „eher selten“ 
mit ihrem Arzt reden konnte, waren psychische, sexuelle Probleme und Probleme in der Partnerschaft.

Unter Clomifen haben sich bei mir sehr schöne Follikel gebildet, aber keine Schleimhaut und kein Schleim. ich soll statt dessen 2 amp. Menogon ab 3.zt nehmen. Mir kommt das etwas viel vor. Mein Arzt diskutiert nicht darüber. Ist das eine übliche Dosis? Keine IVF oder so . (P3; FB 1.txt)

\subsubsection{Beispiel einer erfolgreichen Hilfesuche}

Einige Frauen wandten sich nach erfolgreicher Kinderwunsch-Therapie an das Expertenforum, oft um innen zu danken oder nochmals Fragen zur Schwangerschaft zu stellen. Viele Frauen berichteten, dass das Expertenforum vor allem bei der Orientierung im Gesundheitssystem (,... endlich wurde mir ein Arzt genannt, der mir weiterhilft") und als emotionale Unterstützung sehr hilfreich war.

Eine 24-jährige Schweizerin hatte bei ihrer Suche nach einer Experten basierenden Auskunft im Internet das Expertenforum auf der Website „rund-ums-baby“ gefunden. Davor hatte sie als erstes ihren Frauenarzt um Rat wegen ihrem Kinderwunsch gebeten. Ihre Fragen an die Ärzte des Forums bezogen sich hauptsächlich auf die derzeitige Behandlung und die Auswertung von Untersuchungsergebnissen. Die Antworten der Experten wurden von ihr meist mit ihrem behandelnden Arzt besprochen. Das Expertenforum beurteilte sie wie folgt: „Ich finde es schön, wenn auf alle Fragen eingegangen wird, auch wenn es halt mal einige in der Anzahl sind.......ich denke alle Empfänger sind sehr dankbar dafür auch wenn sie eben etwas länger auf die antworten warten müssen." (P23; FB 190.txt) Aufgrund der Antworten der Experten hatte sie Kontakt mit einem hämatologischen Institut aufgenommen. Die Betreuung durch die dortigen Ärzte beurteilt sie „eher schlecht“, ebenso wie die Aufklärung durch die Ärzte. Obwohl sie sich in dem Institut schlecht beraten fühlte, gab sie an, dass sie mit diesen Ärzten immer über ihre Probleme sprechen zu können. Die medizinische Versorgung durch ihren Frauenarzt beurteilte sie im Durchschnitt mit "gut“.

Liebe Frau Doktor! Ich habe ihnen schon mehrmals über meine Vorgeschichte (2 Spätaborte wegen Plazentainsuffienz und vorzeitige Plazentalösung) berichtet und sie haben mir eigentlich auch großen Mut gemacht es wieder zu versuchen und dafür bin ich ihnen dankbar; denn wer nichts wagt kann auch nicht gewinnen. Nach Unter- 
suchungen hat man erhöhte Gerinnungsfaktoren und eine Genmutation des Prothrombin festgestellt. Nun bin ich wieder einmal in der 14. Woche schwanger und spritze seit der 7. Woche Fraxiparine und nehme dazu noch Aspirin $100 \mathrm{mg}$ plus Magnesium ein. Nach einigen Schwierigkeiten mit dem Spritzen am Anfang (ich hatte unheimlich große blaue blutunterlaufene Flecken) und einer Pilzinfektion kann ich mich im großen und ganzen eigentlich nicht beklagen. Der Hämatologe meinte zwar, dass auch der Haarausfall von den spritzen kommen kann....ich wollte sie eigentlich nur fragen, ob sie spezielle Nebenwirkungen bei der Abgabe des Heparins kennen und vor allem wie verläuft die Abgabe dann bei der Geburt? Nimmt man die Medikamente bis zur Geburt oder hört man vorher damit auf? Kann man trotzdem eine natürliche Geburt anstreben falls keine anderweitige Komplikationen auftreten? Was ist genau wichtig bei der monatlichen Überprüfung der Blutwerte? Welche Untersuchungen sollte der Gynäkologe speziell in meiner Situation beachten? An dieser Stelle möchte ich mich nochmals herzlich bei ihnen bedanken...ihre Unterstützung hat mir bei meiner Trauerarbeit bei der letzten Fehlgeburt sehr geholfen.....liebe Grüße, C. (P23; FB 190.txt) 


\section{Diskussion}

Ziel dieser Studie war es, den Informationsbedarf und die bisherigen Erfahrungen kinderloser Paare in der medizinischen Versorgung indirekt - über die Nutzung eines Expertenforums im Internet - zu beschreiben. Parallel dazu sollte eine direkte Befragung von Nutzern dieses Forums die Stärken und Schwächen internet-basierter Informationen exemplarisch erkennen lassen.

Im Folgenden werden die Befragungsergebnisse zunächst unter methodischen Gesichtspunkten bewertet (6.1) und anschließend unter Berücksichtigung der (auch nach Abschluss der Feldphase veröffentlichten) Literatur diskutiert (6.2). Zum Abschluss (6.3) soll die Qualität dieses und vergleichbarer Internetangebote eingeschätzt werden.

\subsection{Diskussion der Methode}

\subsubsection{Die methodische Triangulation}

Als methodischer Angelpunkt der Arbeit wurde die methodische Triangulation gewählt (Kuzel und Like 1991; Flick 1995). Die Untersuchung erfolgte mit Hilfe folgender Erhebungs- und Auswertungsmethoden:

1. Befragung mit einem standardisierten Fragebogen zu den Nutzungserfahrungen der Besucher eines Expertenforums (5.2);

2. Inhaltsanalytische Auswertung der an die Fragebögen gekoppelten Anfragen an das Expertenforum (5.3); zusätzlich Auswertung einer Stichprobe aus dem gesamten Pool der an das Expertenforum gestellten Fragen und ausgewählter Einzelfälle (5.3.6).

Die Phasen der Erhebung waren hintereinander geschaltet (Phasenmodell, s. Methode; S. 28), zugleich beziehen sich die erhobenen Daten auf unterschiedliche Gegenstandsbereiche (Komplementaritätsmodell): Durch Kombination der Erkenntnisse konnten die beiden Datenmaterialien miteinander verzahnt werden - und standen nicht nur schlicht nebeneinander: Die Auswertung der Anfragen an das Expertenfo- 
rum hatte Einfluss auf die Konzeption des Fragebogens; die Erkenntnisse aus der Befragung flossen wiederum in die Interpretation der Anfragen an das Expertenforum ein. So wurden nicht nur standardisierte Daten in Form von Variablen oder Items erhoben, sondern diese "nackten Zahlen“ konnten durch subjektive Deutungen der Befragten ergänzt, erweitert und durch Kontextinformationen oder Interpretationen angereichert werden (Flick 1995). Durch die methodische Triangulation sollte ein mehrperspektivisches Bild über kinderlose Paare als Nutzer eines Beratungsangebots im Internet entstehen.

\subsubsection{Gütekriterien}

a) Datensicherheit

Da es kaum Literatur über Online-Befragungen und nur wenige Informationen zur Durchführung sozialwissenschaftlich-basierter Untersuchungen im Internet gibt, mussten Methoden entwickelt werden, mit denen es möglich war, große Datenmengen sicher im Internet zu erheben und den kompletten Datensatz vollständig in der abteilungseigenen (nach außen geschützten) Datenbank zu speichern. Durch fingierte (vorher abgesprochene) Anfragen an das Expertenforum und anschließendes Ausfüllen eines entsprechenden Testfragebogens wurde die Datenaufzeichnung einmal pro Woche überprüft. Alle Testfragebögen waren korrekt aufgezeichnet und in der Datenbank gespeichert worden. Das sorgfältige und zuverlässige Datenmanagement, d.h. die sichere Zwischenspeicherung der Daten auf dem Server des Internetangebots und die komplette Übertragung der Daten auf den Abteilungsserver zur anschließenden Bearbeitung in SAS und Altas.ti gelang durch die enge Zusammenarbeit mit der Redaktion und dem Webmaster des Internet-Angebots. Geprüft wurde die Sicherheit des Datenmanagements in der Pilotstudie. Während dieser Zeit traten bei der technischen Verarbeitung der Daten keine nachhaltigen Probleme auf bzw. ließen sich die Probleme durch entsprechend veränderte Kodierung und Programmierung der Fragebögen beheben.

Während der Feldphase kam es zwischen dem 04.09.2001 und dem 08.09.2001 durch einen Programmierungsfehler zu einer fehlerhaften Zuordnung (matching) der Fragebögen und der dazugehörigen Anfragen an das Expertenforum. Dies betraf 22 in der Zeit ausgefüllte Fragebögen. Diese Fragebögen gingen trotzdem - nach positi- 
ver Konsistenzprüfung - in die Bewertung ein. Aufgrund der wöchentlich stattfindenden „Checks“" konnte der Fehler sofort behoben werden.

\section{b) $\underline{\text { Validität }}$}

Durch die hier angewandte Across-methods-Triangulation (s. Methode; S. 28) kann grundsätzlich von einer höheren Validität des Datenpools ausgegangen werden, denn das interessierende Phänomen (Betreuungs- und Informationsbedarf von Patienten) wird durch zwei verschiedene Erhebungsmethoden (Fragebogenuntersuchung und qualitative Inhaltsanalyse der Anfragen an das Expertenforum) erfasst (Jakob 2001). Diese höhere Validität bezüglich des untersuchten Gegenstandes kann aber nur dann geltend gemacht werden, wenn die unterschiedlichen Datenpools (Ergebnisse der Fragebogenuntersuchung und der Inhaltsanalyse) verknüpft werden (Tudiver et al. 1991, Jakob 2001). Durch das parallele Auswerten und Vergleichen sollte jede Methode in sich geprüft werden. Dies galt vor allem für die schwer zu standardisierende inhaltsanalytische Auswertung der Anfragen an das Expertenforum. Auf der anderen Seite wurden die Ergebnisse des relativ starren (standardisierten) Fragebogens durch die Ergebnisse aus der qualitativen Studie erweitert, differenziert und bereichert.

\subsubsection{Quantitative Erhebung: Online-Befragung}

Das Instrument der Befragung im Internet unterliegt, wie auch das persönliche oder telefonische Interview, allgemeinen Fehlerquellen: Auswahl der Stichprobe, Fragebogenentwicklung und Befragungssituation. Die Befragung ist eine reaktive Erhebungsmethode (Theobald 2000): Die befragte Person muss auf irgendeine Weise auf einen artifiziellen Input reagieren und verhält sich damit anders, als sie es ohne Befragung getan hätte. Die Befragung ist prinzipiell fehlerbehaftet (Sudman und Bradburn 1974). Für die Ergebnisse ist deshalb das Risiko störender, methodisch bedingter Effekte abzuschätzen.

Aufgrund der Neuheit der Erhebungsmethode ist der Wissensstand über Befragungen im Internet gering. Die Diskussion methodischer Probleme greift daher auf Erkenntnisse über erprobte Formen der Erhebung, z. B. Telefon- und Face-to-faceInterviews, zurück. 
Die Vorteile der online-vermittelten Befragung bestehen aus der weitgehenden Automatisierung bei Durchführung, Erhebung und Datenverarbeitung. Die Daten sind daher schnell verfügbar. Ein weiterer Vorteil von Online-Befragungen ist, dass sich hohe Fallzahlen in relativ kurzer Zeit mit vergleichsweise geringem finanziellen Aufwand generieren lassen (Bandilla et al. 2001).

Das sonst bei dieser Art von Erhebungen größte Problem - die Motivierung der aktiven Teilnahme (Baker 1996) - bestand bei dieser Umfrage nicht: Die Besucher des Expertenforums waren durch ihre direkte Betroffenheit hochmotiviert, an der Befragung teilzunehmen. Dies spiegelt sich in der Teilnahmerate von $84,4 \%$ wider. Schnell et al. (1997) begründen die relativ hohen Teilnehmerzahlen bei Online-Befragungen durch die Neuartigkeit der Erhebung, die den gewünschten Teilnehmerkreis durch eine andere (,frische“) Art erreichen und aktivieren kann.

Der Entschluss, den Fragebogen im Anschluss an eine Anfrage an das Expertenforum zu positionieren, ist mit dem Risiko der Verunsicherung der entsprechenden Besucher des Expertenforums behaftet. Die dadurch möglicherweise höhere Verweigerungsrate wurde zugunsten einer permanenten Präsenz des Fragebogens und der daraus erhofften wahrscheinlich höheren Teilnehmerzahl in Kauf genommen. Über die Anzahl der Personen, die die Teilnahme verweigerten, indem sie das Internet oder die spezielle Website ohne weitere Information verließen, lässt sich nur spekulieren. Aus diesem Grund muss die hohe Antwortrate von $84,4 \%$ gegebenenfalls relativiert, auf alle Fälle aber mit Vorsicht interpretiert werden.

Nachteilig für das Ziel repräsentativer Studien ist die noch relativ geringe Nutzung des Internets. Nach Untersuchungen des Statistischen Bundesamtes verfügten im Januar 2001 erst ca. 53\% der Haushalte in Deutschland über (mindestens) einen Computer. Bundesweit hatten rund $27 \%$ der Haushalte einen Internetzugang (Statistisches Bundesamt 2002).

Für die vorliegende Untersuchung ist diese Verzerrung unerheblich, da sich die Aussagen bewusst nicht auf die "Gesamtbevölkerung", sondern nur auf die Grundgesamtheit der Internet-Nutzer beziehen. Die in dieser Untersuchung verschiedentlich 
geäußerte Kritik an der medizinischen Betreuung sollte somit auch nicht auf alle Kinderwunsch-Patienten generalisiert werden.

Die unkontrollierbare Befragungssituation wirkt sich nachteilig auf die Reliabilität der Ergebnisse aus (Fuchslocher und Fritz 1992; Eysenbach 2002), vor allem durch nicht-systematische Falsch-Angaben (der Proband gibt einfach "irgendetwas“ an). Mangelnde "Sanktionierbarkeit" sowie geringe Transparenz und Überprüfbarkeit der Ergebnisse begünstigen solches Verhalten. Auf der anderen Seite kann der Interviewer die Beantwortung nicht beeinflussen (kein Interviewer-Bias; Fuchslocher und Fritz 1992). Aus diesem Grund sind aber auch die Befragungsabbrüche durch Verständnisprobleme, die nicht durch sofortige (persönliche) Hilfe gelöst werden können, und Zeitprobleme bei Internetbefragungen relativ zahlreich (Theobald 2000). Durch die unkontrollierte und anonyme Interviewsituation während der Online-Studie sind keine zuverlässigen Angaben zu Mehrfach-Antwortern möglich. Diese hätten durch IP-Nummern ${ }^{1}$ rückverfolgt werden können; aufgrund des Datenschutzes kam diese Art der Teilnehmerkontrolle nicht in Frage. Die Kontrolle selbst wäre auch nur bedingt aussagefähig, da ein und dieselbe Person von verschiedenen PCs Anfragen stellen könnte.

\section{Feldzeit}

Die Feldzeit der Online-Befragung war untypisch lang (ADM 2001). Ursächlich dafür war, dass der Pool der Fragesteller im Expertenforum über gewisse Zeiträume immer von denselben Personen gebildet wurde und sich nur langsam änderte. Viele Forumteilnehmer stellten für eine bestimmte Zeit mehrmals Fragen im Expertenforum und bekamen demnach mehrfach den Fragebogen angeboten (632/1305). Deshalb wurde am Anfang des Fragebogens gefragt, ob der Fragebogen schon einmal ausgefüllt worden war. Die Ausdehnung der Feldzeit erschloss dann allmählich die gewünschte Stichprobengröße.

\footnotetext{
${ }^{1}$ Die IP (Internet Protokoll)-Nummer ist die Netzadresse eines Computers im Internet. Sie macht somit eine eindeutige Zuordnung der Teilnehmer im World Wide Web möglich.
} 


\section{Stichprobe}

Die Einbettung der Studie in ein spezielles Internetangebot für kinderlose Paare garantierte eine zielgruppenspezifische Ansprache der Studienteilnehmer. Trotzdem kann es zu in der Größenordnung unbekannten Selbstselektionseffekten kommen:

- Die betroffenen Personen müssen die Umfrage auf der Seite „rund-ums-baby.de“ finden bzw. sie müssen sich entschließen, eine Frage an das Expertenforum zu stellen;

- Die Befragten müssen aktiv über ihre Teilnahme an der Studie entscheiden;

- Die Teilnehmer müssen bereit sein, für die durch das Ausfüllen des Fragebogens ggf. entstehenden Kosten (z. B. Provider- und Verbindungsgebühren) selbst aufzukommen.

Ein weiterer Selbstselektionseffekt war, dass vornehmlich „Unzufriedene“ bzw. mit der bisherigen Therapie/Diagnostik gescheiterte Paare sich an das Internet wandten. Die Kritik dieser Personen an der Medizin gilt daher nicht für die gesamte Reproduktionsmedizin.

Aufgrund dieser Selektionseffekte, vor allem aufgrund des Themas des Expertenforums weicht die Stichprobenzusammensetzung in wichtigen Parametern (Geschlecht, Familienstand und Ausbildungsniveau) von der Gesamtbevölkerung in der befragten Altersgruppe ab: Sowohl der Anteil der Männer als auch der Anteil der Single-Haushalte ist deutlich unterrepräsentiert. Überdies ist in der Umfrage eine deutliche Verzerrung (bias) in Richtung „höheres Bildungsniveau“ zu verzeichnen.

Darüber hinaus hängt die Wahrscheinlichkeit, den Fragebogen „im Netz“ zu finden, von der Nutzungsintensität des Internets ab, d.h. es ist ein Bias in Richtung Vielnutzer des Internets anzunehmen. Des Weiteren dürften verstärkt Frauen und Männer mit Internetzugang am Arbeitsplatz unter den Teilnehmern der Studie zu finden sein: Die Auswertung nach Wochentagen ergab, dass die meisten Anfragen Montag bis Mittwoch eingingen (54,0\%), um dann bis zum Wochenende stetig abzufallen. Am Wochenende nahm die Anfragefrequenz stark ab $(17,8 \%)$. Es zeigte sich, dass die 
meisten Anfragen innerhalb der üblichen Bürokernzeiten - zwischen 9.00 Uhr und $15.00 \mathrm{Uhr}$ - registriert wurden (Abbildungen 6 und 7$)^{1}$.

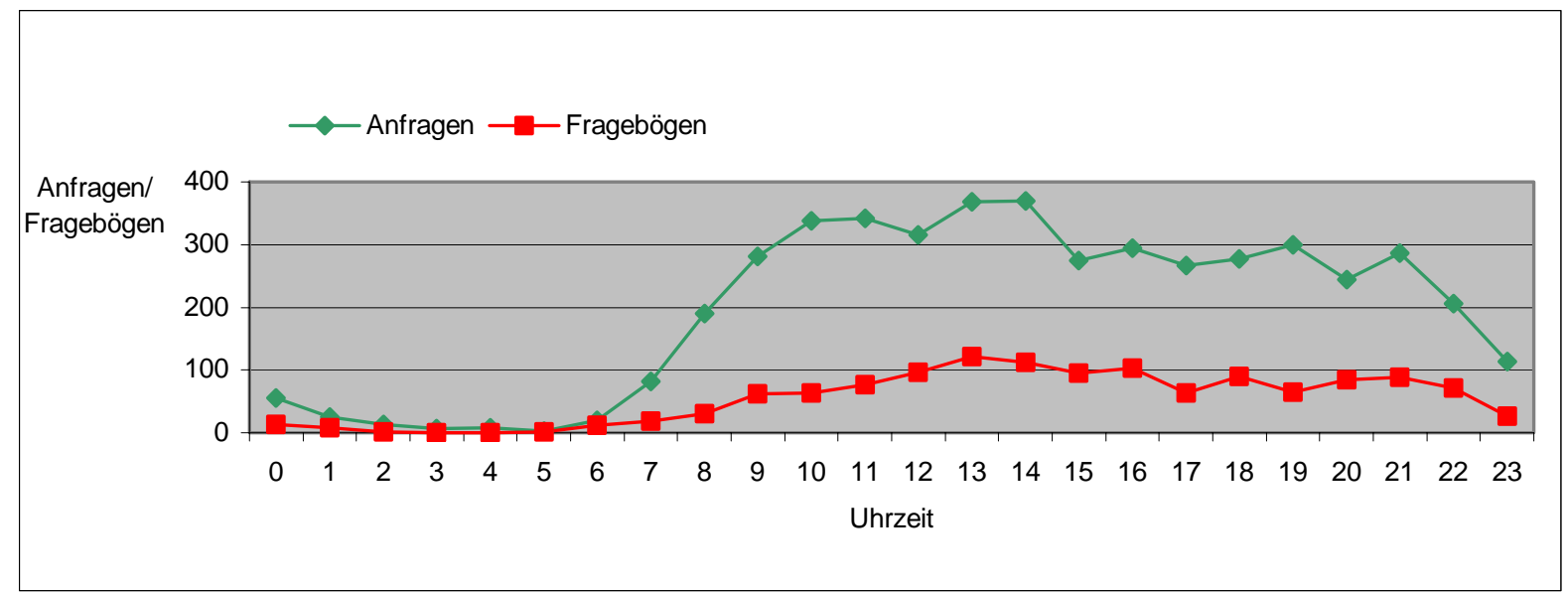

Abbildung 6: Uhrzeit Anfragen und Fragebogenrücklauf

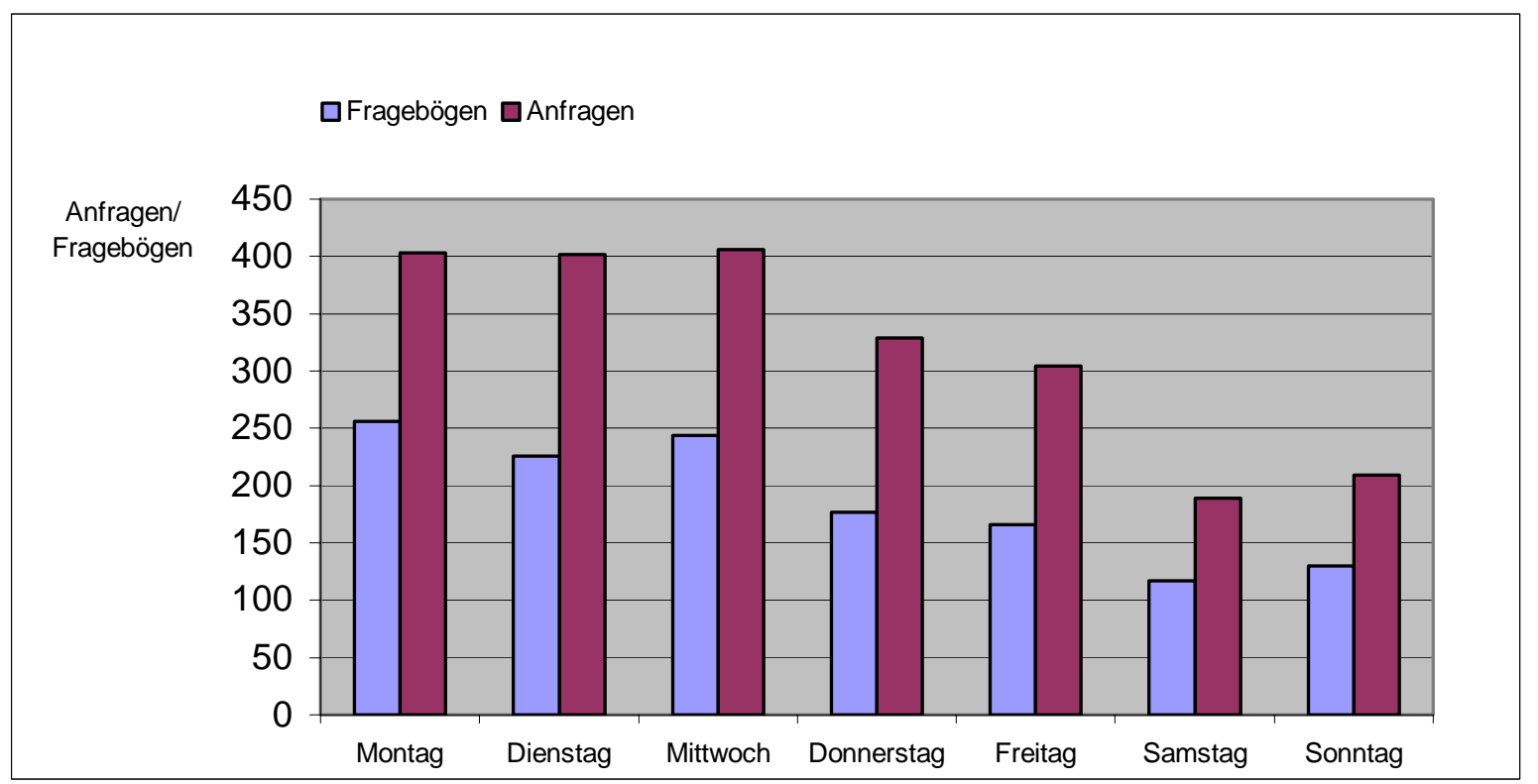

Abbildung 7: Anfragen an das Expertenforum und Beantwortung der Fragebögen; Wochentage

Der Effekt der „sozialen Erwünschtheit“ ist in einer schriftlichen Befragung als vergleichsweise gering einzustufen (Frey et al. 1990). Durch die Anonymität der Befragten im Internet ist dieser Effekt noch geringer. Allerdings erwarten die Befragten von dem Expertenforum Informationen, die sie von ihrem behandelnden Arzt nicht erhielten oder „absichern“ wollen. Ein gewisser Druck zu positiver Bewertung des Expertenforums ist daher nicht auszuschließen - aus der Angst vor Informationsverweige-

\footnotetext{
${ }^{1}$ Für die Diskussion stellte der Webmaster der Seite „rund-ums-baby.de“ diese Daten zur Verfügung.
} 
rung bei „unbotmäßigem Verhalten“. Eine entsprechend gegenteilige Versicherung unsererseits konnte dieser Angst wahrscheinlich nur bedingt entgegenwirken. Umgekehrt hat die (anonyme) Online-Befragung Patienten gynäkologischer und hausärztlicher Praxen bzw. reproduktionsmedizinischer Zentren vermutlich motiviert, die Qualität medizinischer Betreuung differenzierter und offen kritisch zu bewerten. Zusätzlich konnten der Betreuungs- und Informationsbedarf von Personen ohne aktuellen Arztkontakt miteinbezogen werden.

\subsubsection{Inhaltsanalytische Auswertung}

Gegenüber der statistischen Auswertung der Online-Befragung beruhte der qualitative Ansatz auf nicht-numerischem Material aus den Anfragen an das Expertenforum, das interpretativ ausgewertet wurde. Dieses Material ist "reichhaltiger", da es mehr Details enthält als ein quantitativer Messwert (Bortz und Döring 2002, S. 296). Zusätzlich wurden aber auch in der qualitativen Auswertung Quantifizierungen in Form von Frequenzanalysen (quantitative Inhaltsanalyse) durchgeführt; es wurden bestimmte formale oder inhaltliche Elemente des Materials ausgezählt. Die Inhaltsanalyse wurde also im Forschungsprozess sowohl zur Auswertung als auch zur Datenerhebung genutzt.

\section{Gütekriterien}

Die Gütekriterien der quantitativen Messung (Objektivität, Reliabilität und Validität) werden in modifizierter Form auch in qualitativen Studien verwendet. Die Validität ist auch hier das wichtigste Gütekriterium der Datenerhebung und -auswertung:

Um eine Beeinflussung des Inhalts einer Anfrage an das Expertenforum durch die Teilnahme an der Studie zu vermeiden, wurde der Fragebogen erst nach dem Absenden der Anfrage eingeblendet. Die Fragen wurden demnach unbeeinflusst und authentisch an das Expertenforum gestellt.

Trotz der schon durch die Across-methods-Triangulation relativ hohen Validität (Titscher et al. 1998; Jakob 2001) wurde auch innerhalb der qualitativen Auswertung versucht, die Auswertung und Interpretation durch folgende Maßnahmen transparent und objektiv zu gestalten: 
1. Deduktiv und induktiv erstellte Kodierliste und -regeln zur Auswertung;

2. Beispiele zur Anwendung der unter 1) genannten Kodierregeln;

3. Regelmäßige und wiederholte Arbeitsbesprechungen zur Interpretation.

Gemäß den Kriterien für qualitative Sozialforschung (Strauss 1991) beanspruchen die Ergebnisse nur Gültigkeit für die Situation von Personen (Paare), die das Internet nutzen. Sie sind nicht unmittelbar auf die Erfahrungen von Patientinnen und Patienten übertragbar, die sich zur aktuellen Behandlung in den ambulanten Praxen oder spezialisierten Einrichtungen befinden.

\subsection{Diskussion der Ergebnisse}

\subsubsection{Stichprobe}

In Deutschland nutzen deutlich mehr Männer (61\%) als Frauen (39\%) das Internet (Nielsen/NetRatings 2002 b). Im Gegensatz dazu sind in unserer Studie kaum Männer unter den Fragestellern des Expertenforums (0,8\%). Ähnliches wurde in früheren Online-Studien zu spezifischen Nutzungsverhalten in "health communities" beschrieben (Diaz et al. 2002; Houston et al. 2002): Zum Beispiel berichteten Epstein et al. (2002), dass in ihrer Internet-Umfrage über die emotionalen Konsequenzen bei der Nutzung spezieller Internet-Angebote für ungewollt Kinderlose über $99 \%$ der Studienteilnehmer Frauen waren. Dies könnte zunächst daran liegen, dass Frauen das Internet häufiger zur Suche nach Gesundheitsinformationen nutzen als Männer (Taylor MRG et al. 2001). Entscheidender ist sicherlich, dass Frauen in der Wahrnehmung medizinischer Probleme und in Behandlungsentscheidungen, vor allem in der Reproduktionsmedizin, deutlich stärker involviert sind und sich für das „Informationsmanagement" verantwortlich fühlen:

So gelten Frauen als der engagiertere Partner in der Infertilitätstherapie: „Motor des Behandlungswunsches der Kinderlosigkeit" nennt sie Onnen-Isemann (2000). Meistens sind es die Frauen, die sich als erstes für die reproduktionsmedizinische Behandlung interessieren und den Partner zur Begleitung/Behandlung überzeugen. Nur 
wenige Männer übernehmen den aktiven Part (Berg BJ et al. 1991; Onnen-Isemann 2000). Ein weiterer Grund für die Initiative der Frauen könnte das bei Greil et al. (1988) beschriebene Erleben von Frauen sein, ihrer Rolle, insbesondere der Erfüllung der Idealnormen der Gesellschaft (Gründung einer Familie, Mutterrolle) nicht gerecht zu werden. Schließlich empfinden Frauen das gemeinsame Erleben des gleichen Schicksals - die Öffnung nach außen zu einer „eingeweihten“ Freundin oder anderen Betroffenen - als Hilfe (Berg BJ et al. 1991; Stanton et al. 1991; Schröter et al. 1998). Dies könnte ein weiterer Grund für die starke Präsenz weiblicher Besucher im Expertenforum bzw. weiblicher Studienteilnehmer sein.

Laut Nielsen/NetRatings (2002 b) und EORG (2003) sind Personen mit hohem Bildungsniveau die Hauptnutzer des Internets. In unserer Studie waren Personen mit höherem Bildungsgrad ebenfalls überrepräsentiert. Hierin könnte sich gleichzeitig ein Zusammenhang zwischen Bildungsstand und Kinderlosigkeit ausdrücken: Laut Huinink und Brähler (2000) schieben Menschen mit höherem Bildungsstand eine (später) gewünschte Schwangerschaft häufiger auf - mit dem Risiko der altersbedingten Sterilität (Wischmann et al. 2000).

Auf die Frage nach dem Einkommen wurde in der Studie bewusst verzichtet, um einen frühzeitigen Abbruch der Beantwortung des Fragebogens zu vermeiden. Aus der hohen Zahl gutausgebildeter Personen in der Stichprobe und den Nutzungsspitzen des Expertenforums zu Arbeitszeiten (Abbildung 6; S. 67) kann man folgern, dass in der Studie relativ viele berufstätige (und auch gutverdienende) Personen vertreten sind. Dies entspräche den Ergebnissen der Untersuchungen von Diaz et al. (2002): Patienten, die das Internet auf der Suche nach medizinischen Informationen nutzten, waren im Durchschnitt besser ausgebildet und hatten ein wesentlich höheres Jahreseinkommen als eine Vergleichsgruppe, die das Internet nicht nutzte.

\subsubsection{Signalisierter Hilfebedarf}

Viele der von uns befragten Personen äußerten eine gewisse Frustration über den bisherigen Krankheitsverlauf, über ausbleibende Behandlungserfolge oder über ihren behandelnden Arzt. Trotzdem ergab die Umfrage, dass die Mehrzahl der Befragten mit ihren Ärzten zufrieden war: Vor allem die medizinische Betreuung wurde überwiegend positiv gewertet, etwas schlechter die Aufklärung und die Bereitschaft zu ausführlichen Gesprächen. Vergleichsweise viele Personen (41\%) berichteten über 
Defizite in der hausärztlichen Betreuung bei Kinderlosigkeit. Ein Betreuungsbedarf kinderloser Paare in der hausärztlichen Versorgung und ein Wunsch dieser Patienten nach mehr Betreuung durch den Hausarzt wurde schon in der Studie von Schröter et al. (1998) aufgezeigt. Dabei könnte gerade der Hausarzt Patienten/Paare mit (bisher) unerfülltem Kinderwunsch in verschiedenen Stadien unterstützen und beraten (Himmel et al. 1997): (1) im Vorfeld einer reproduktionsmedizinischen Behandlung als neutraler Informant über Chancen und Risiken (Schröter et al. 1998), (2) während der Behandlung als emotionale Stütze, ggf. auch als Mittherapeut und (3) nach Abschluss als kontinuierlicher Begleiter, insbesondere wenn sich der Kinderwunsch nicht realisieren ließ. Gerade dann ist die längerfristige Unterstützung durch Spezialisten und Kliniken nicht zu erwarten und die familienmedizinische Bedeutung des Hausarztes erwünscht (Ittner et al. 1997, 1998). Diese Aufgabe wird bisher aber nicht optimal erfüllt (Kochen et al. 2001).

Ein Defizit an Aufklärung und Zeit für ausführliche Gespräche spiegeln die vielen Anfragen an das Expertenforum zur derzeitigen Therapie sowie zu den Untersuchungstechniken und -ergebnissen wider. Trotz der guten Bewertung der behandelnden Frauenärzte und Ärzte in Kinderwunsch-Zentren wird in den Anfragen häufig die mangelnde persönliche Betreuung kritisiert. Diese Diskrepanz ist möglicherweise schon eine indirekte Wirkung des Expertenforums: Die Informationen können auf viele Anfragesteller angstreduzierend wirken, in dem z.B. die Therapieansätze der behandelnden Ärzte bestätigt und nochmals genau erklärt wurden. Dies könnte die nachträglich - höhere allgemeine Zufriedenheit mit der ärztlichen Versorgung in der Fragebogenumfrage begründen. Nach Köhler und Eysenbach (2002) korrelieren angstreduzierende Wirkung von Web-basierten Informationsprogrammen und Zufriedenheit mit der medizinischen Versorgung: Die Patienten fühlten sich besser unterstützt und hatten einen niedrigeren Angstscore. In dieser Studie waren ebenfalls vor allem Befragte, die die Antworten der Experten des Kinderwunschforums mit ihren behandelnden Ärzten besprachen, mit der ärztlichen Versorgung zufriedener $(80,1 \%)$ - im Vergleich zu 64,2\% der Befragten, die inre Informationen nicht in das Arztgespräch einbrachten.

58 Studienteilnehmer drückten in ihren Anfragen explizit ihre Unzufriedenheit mit dem behandelnden Arzt aus. Vielfach waren sie über das Vorgehen des Arztes irritiert. Die Unsicherheit und das Misstrauen waren, nach Angabe vieler Frauen, häufig 
Folge unzureichender Aufklärung über die Teilschritte der Therapie. Zum Teil schienen die Frauen durch Artikel in Zeitschriften oder durch Veröffentlichungen im Internet, deren Lektüre ohne medizinische Vorbildung oftmals irreführend ist, zusätzlich verunsichert. Auch hier dürften die Experten des Forums „Kinderwunsch“ durch ihre oft ausführlichen Antworten zur Klärung viele Unsicherheiten beigetragen haben.

Die in unserer Studie befragten Besucherinnen und Besucher des Expertenforums bestätigten die in der Literatur beschriebenen psychischen Probleme durch ungewollte Kinderlosigkeit, wie Ängste, Verlusterfahrungen, Unverständnis oder Erfolgsdruck und schließlich die Belastung durch fehlendes ärztliches Verständnis und durch das reproduktionsmedizinische Verfahren selbst und die negativen Auswirkungen auf die Partnerschaft (Hölzle 1990; Strauß et al. 1991; Schröter 1998; Henning und Strauß 2002). Aus der qualitativen Analyse gehen vor allem die subjektiv empfundene Belastung und die Hilflosigkeit während der Behandlungszeit hervor. Nur ein relativ geringer Anteil (ca. 20\%) an Befragten befand sich in psychologischer Betreuung; auch dies ist ein in der Literatur nicht unbekanntes Ergebnis (Ningel und Strauß 2002).

\subsubsection{Nutzung des Expertenforums}

Am häufigsten hatten die Expertenforum-Besucher das Interesse, tiefer gehende Informationen über die Kinderlosigkeit zu erhalten. Ein relativ großer Teil der Besucher erfragte Informationen zur Ursache und zum Pathomechanismus der eigenen zur Unfruchtbarkeit führenden Erkrankung; Informationen bzw. Interpretationen von Laborwerte waren ebenfalls ein häufiges Thema. Vielen Befragten diente das Expertenforum als Informationsquelle für eine „zweite Meinung“ zu Behandlungsmethoden und Untersuchungsergebnissen. Nach einer europaweiten Studie der Boston Consulting Group (BCG, 2001) suchten 52\% der befragten Personen im Internet nach aktuellen Studienergebnissen, 42\% nach Expertenrat und 40\% nach Hintergrundinformationen zu ihrer jeweiligen Erkrankung.

Eysenbach und Diepgen (1999 a) analysierten die Gründe und die Art der gestellten Patientenfragen in dermatologischen Gesundheitsportalen. Die Mehrzahl der Befragten nutzte dieses Gesundheitsportal um eine zweite Meinung zu ihrer Erkrankung zu erhalten. Die Patienten suchten nicht allgemeine Auskünfte im Web, sondern spezielle Informationen zu bestimmten Krankheiten. Begründet wurde dies damit, dass im 
Zentrum der Behandlung der Arzt-Patienten-Kontakt stehe; und genau nach dieser individuellen und an ihre aktuelle Situation angepassten „Behandlung“ suchten die Patienten auch im Internet (BCG 2001).

Dieses Bedürfnis nach individuumsorientierten Informationen kann offensichtlich das Expertenforum „rund-ums-baby.de“ befriedigen, da die Experten jede E-Mail individuell beantworten. Ein Vorteil gegenüber der reinen Telekonsultation zwischen Experten und Anfragesteller hinausgehender Vorteil, ist die Zugänglichkeit der Anfragen und Antworten für alle Besucher des Forums - als gute Ergänzung zur reinen Informationssuche im Internet.

Neben der fachkompetenten Auskunft erhofften sich viele Nutzer auch eine emotionale Unterstützung von den Experten: So äußerten in unserer Studie viele Frauen in ihren Anfragen und im Fragebogen den Wunsch nach mehr Zuwendung seitens der Experten und mehr Individualität und Einfühlungsvermögen bei der Beantwortung der Fragen. Ob das Expertenforum als emotionale Stütze oder Begleiter dienen kann, ist nur schwer einzuschätzen. Der Experte könnte eine erhebliche Bedeutung zur Klärung der eigenen Situation bzw. zur Orientierung im Gesundheitswesen haben. Er könnte lenkend, „fixierungslösend“ oder emotional stabilisierend wirken. Die Forumexperten waren meist ergänzend zur Tätigkeit des behandelnden Arztes tätig. Durch Erklären von Therapien, Eingehen auf spezielle Fragen, die nicht mit dem Arzt geklärt wurden, konnte nach Angaben einiger Kinderwunschpaare ein Teil ihrer Ängste und Unsicherheiten genommen werden. Durch die Kommunikation mit anderen „Leidensgenossen" in Chatrooms der Seite "rund-ums-baby.de" oder durch das Lesen der im Expertenforum veröffentlichten Fragen der Betroffenen und Antworten der Experten können viele Hilfesuchende das greifbare Gefühl bekommen, dass ihre Kinderlosigkeit kein Einzelschicksal ist, sondern ein Problem, das sie mit vielen Paaren teilen. Auch dies kann vielen Betroffenen neue Hoffnung und Mut geben, eine Therapie zu beginnen, eine schon begonnene Therapie fortzusetzen oder auch eine informierte und für sich selbst akzeptierte Entscheidung zum Verzicht auf eine Therapie zu treffen. 


\subsubsection{Bewertung des Internetangebots}

Die meisten Besucher des Expertenforums fanden die Seite „rund-ums-baby.de“ durch Zufall beim Suchen in verschiedenen Suchmaschinen (46,4\%). Dies wurde auch in anderen Studien zur Internetnutzung festgestellt: Laut einer Umfrage von BCG (2001) suchen Patienten als erstes in Suchmaschinen; seltener geht ihre Suche von speziellen „Gesundheitsportalen“ aus. Fast 15\% unserer Befragten wandten sich wegen ihrer Kinderlosigkeit an das Expertenforum als „ersten Ansprechpartner“. Damit war das Expertenforum nach dem Frauenarzt (75\%) ein recht wichtiger Ansprechpartner auf der Suche nach Hilfe bei unerfülltem Kinderwunsch. Der Hausarzt spielte als Berater bzw. erster Ansprechpartner bei Kinderlosigkeit mit ca. 2\% kaum eine Rolle. Aktuelle gesundheitspolitische Tendenzen, den Hausarzt zum Gatekeeper für den Eingang zum Gesundheitssystem zu machen, scheinen bei ungewollter Kinderlosigkeit zumindest für diese Gruppe der Befragten weniger bedeutsam zu sein.

Die Mehrzahl der Fragesteller war mit der Arbeit der Kinderwunsch-Experten zufrieden. Auch in der inhaltsanalytischen Auswertung der Anfragen wurde der „Konsiliardienst" der Experten überwiegend positiv bewertet. Die wenigen negativen Anmerkungen bezogen sich auf gelegentlich lange Beantwortungszeit und die manchmal unzureichenden bzw. unverständlichen Antworten der Experten. Diese Kritik schränkten die Frauen oftmals selbst ein, insoweit sie ihre eigene Ungeduld als ursächlich sahen. Die hohe Zufriedenheit sowohl in der Fragebogenumfrage und in der inhaltsanalytischen Auswertung der Anfragen lässt sich aber vorläufig nur bedingt als Qualitätsmerkmal des Expertenforums verwenden, da es wahrscheinlich, aber nicht sicher ist, dass die Kinderwunsch-Paare nach der Beratung wirklich besser informiert waren bzw. dass das Expertenforum eine wirkliche Hilfe für die kinderlosen Paare darstellte. An dieser Stelle ist auch ein Forschungsbedarf für die Zukunft markiert.

Hinsichtlich der additiven Beratung der Patienten - zusätzlich zum Gespräch mit dem behandelnden Arzt - durch Online-Konsiliardienste, sind kaum Nachteile für den Patienten zu entdecken: Die Patienten können sich leichter als früher umfassend über die verschiedenen Behandlungsmöglichkeiten und -alternativen informieren (,information therapy“, Bader und Braude 1998) und auf diesem Weg aktiv in den therapeutischen Entscheidungsprozess integriert werden („shared decision-making“, Gerber 
und Eiser 2001). In vielen Studien zur Veränderung der Arzt-Patient-Beziehung im Zeitalter der Telekommunikation sowie auch in vielen Anfragen dieser Studie zeichnete sich ab, dass Patienten bzw. kinderlose Paare Diagnosen oder Therapievorschläge nicht nur hinnehmen, sondern aktiv in den Entscheidungsprozess integriert werden und umfassend informiert werden wollten (Köhler und Eysenbach 2002; Anderson et al. 2003).

Vorteilhaft ist die Internetberatung vor allem für Patienten im ländlichen Raum bzw. in Kleinstädten - über die Hälfte unserer Befragten. Gerade für diesen Personenkreis dürfte es schwierig sein, regelmäßig einen Spezialisten oder eine Spezialeinrichtung aufzusuchen, um fachlich angemessen beraten zu werden. Weit stärker als für Deutschland dürfte diese Aussage für Länder mit vielen gering bevölkerten Zonen, wie z. B. Australien, Norwegen usw. gelten. Andererseits könnte die Arbeitsüberlastung vieler Ärzte dazu führen, dass nicht genügend Zeit vorhanden ist, um sich eingehend mit den neuesten Methoden eines Fachgebiets auseinander zu setzen. Aus Zeitmangel können ausführliche Gespräche nur selten während der Sprechzeiten realisiert werden; Telefonate stehen ebenfalls unter Termindruck (Koubenec 2001; Swoboda 1997). Ein Internet basierter Auskunftsdienst, wie das Expertenforum, könnte hier für räumliche und zeitliche Entzerrung sorgen.

Problematisch wird die Beratung über das Internet, wenn sie der einzige Kontakt zum Gesundheitssystem bleibt und die persönliche Beziehung zwischen Arzt und Patient teilweise oder gänzlich einschränkt bzw. ersetzt. Das Fehlen einer direkten ArztPatienten-Interaktion erhöht das Risiko falscher Diagnosen und Fehlbehandlungen, da für die Spezifität bestimmter zur Kinderlosigkeit führender Erkrankungen in starkem Maß empathische Informationen und implizite Äußerungen relevant sind (z. B. bei Potenzstörungen aufgrund von Problemen in der Partnerschaft). Darüber hinaus kommt Baur (2000) in einem Review über den Einfluss von E-Mail und Internet auf die Arzt-Patienten-Kommunikation zum Schluss, dass E-Mail-Konsultationen die schon bestehende Tendenz von Ärzten unterstütze, Gespräche technisch-informell zu führen und weniger auf psychosoziale Aspekte zu achten (s.a. Schmidt und Koch 2003).

In verschiedenen Studien wird als weiteres Problem die allgemeine Verunsicherung und Angst über die Vertraulichkeit und den Datenschutz genannt. Gemäß einer Un- 
tersuchung von Moormann und Van der Lei (1999) stieg dieses Misstrauen in den letzten Jahren in der Allgemeinbevölkerung stetig an. Trotz der steigenden Bedenken gegenüber der Datensicherheit im Internet zeigten sich die Besucher des Expertenforums in vielen Anfragen erstaunlich offen. Das Konzept des hier untersuchten Expertenforums scheint den Frauen genug Anonymität zu bieten, um auch intime Informationen mitzuteilen.

In unserer Studie wurde nur ein geringer Teil der Befragten (1,3\%) von ihren Ärzten auf die Möglichkeit eines „Konsiliardienstes“ im Internet aufmerksam gemacht. In der Studie von O'Connor und Johanson (2000) wurden ebenfalls nur 4\% der befragten Patienten in gastroenterologischer Behandlung durch ihre Ärzte an das Internet verwiesen. Zu ähnlichen Ergebnissen kam eine Telefonumfrage von Cyberdialogue (1998). Die geringe Zahl an Ärzten, die Internetseiten oder bestimmte Internetdienste empfehlen, steht im auffälligen Kontrast zur eher technikfreundlichen Einstellung vieler Patienten: Taylor MRG et al. (2001) stellten fest, dass über $90 \%$ der von ihnen in Befragten interessiert waren, die Website ihres behandelnden Arztes bzw. die von den Ärzten empfohlenen Websites zu besuchen oder mit ihrem Arzt in E-Mail Kontakt zu stehen.

Ärzte stehen der Arzt-Patienten-Kommunikation über das Internet eher skeptisch gegenüber: Laut einer Umfrage von Cyberdialogue (1998) hielten nur 39\% der Ärzte das Internet für eine geeignete Informationsquelle bei Gesundheitsfragen; nur 9\% der von Forrester Research (2002) befragten Ärzte waren bereit, mit ihren Patienten über E-Mail in Kontakt zu stehen. Patt et al. (2003) stellten fest, dass Ärzte zwar die Kommunikation über das Internet vor allem für die Betreuung chronisch Kranker als Bereicherung empfinden; die technische Umsetzung im Praxisalltag schätzen sie aber als schwierig ein, da das Internet zum einen nicht für jeden Patienten als Kommunikationsmittel geeignet sei und es noch an Sicherheits- und Qualitätsstandards mangelte. Von Anderson et al. (2003) befragte Ärzte befürchteten, dass die Patienten sich mit ernsthaften Erkrankungen verstärkt dem Internet zuwenden, anstatt einen Arzt zu konsultieren.

Trotz der kritischen Haltung der Ärzte gegenüber dem Internet, steigt die Zahl der Patienten, die das Internet als Informationsquelle nutzen stetig an - schon allein deshalb, weil die Zahl der Haushalte mit Internetanschluss jährlich steigt und da- 
durch die technische Voraussetzung, im Internet problemlos von zuhause aus nach Informationen zu suchen. Ein weiterer Grund könnte der Vertrauensverlust in die ärztliche Versorgung sein: Insbesondere unter dem Kostendruck im Gesundheitswesen sind immer mehr Patienten besorgt, nicht die optimale Therapie zu erhalten (Anderson et al. 2003). Schließlich machten die in unserer Studie Befragten auf Zeitmangel in der medizinischen Versorgung aufmerksam: sie würden sich daher nicht ausreichend von ihren Ärzten aufgeklärt und informiert fühlen und sich alternativen Informationsquellen, wie dem Expertenforum, zuwenden. Vor allem in diesen Fällen konnte der Konsiliardienst der Experten auf der Seite „rund-ums-baby“ zu einer verbesserten Patienteninformation bzw. -versorgung beitragen.

Wenn Ärzte darauf vorbereitet sind, mit alternativen Therapievorschlägen aus dem Internet konfrontiert zu werden und diese - soweit medizinisch vertretbar - zu akzeptieren (Gerber und Eiser 2001), könnte sich das Zusammentreffen von Patient und Arzt oftmals produktiver gestalten (Sänger 1999). Im optimalen Fall werden Ärzte ihre Patienten bei der Informationssuche unterstützen (z. B. Empfehlen geeigneter WebAdressen).

\subsection{Ausblick}

Neue Internet- und Netzwerktechnologien werden herkömmliche Kommunikationsstrukturen im Gesundheitswesen stark verändern: Im Gegensatz zu den Printmedien bietet das Internet eine bi-direktionale Kommunikation, die durch interaktive Rückkopplung sowohl eine massenmediale als auch eine individuumsorientierte Informationsweitergabe ermöglicht. Zwei wichtige Anwendungen sind (1) Netzwerke zwischen den behandelnden Ärzten und Spezialisten bestimmter Fachrichtungen (Telekonsultation; Larkins 2003) und (2) elektronische Patientenakten, die von Patienten, Ärzten, Apothekern und anderen Fachleuten des Gesundheitssystems eingesehen und ergänzt werden (C-Connectivity; Waegemann 2001). Diese Anwendung sollen die Qualität und die Effizienz medizinischer Leistung verbessern (Richardson 2002, Eysenbach und Diepgen 1999 b).

Ein weiterer medizinischer Anwendungsbereich des Internets ist das E-Commerce: Obwohl der Anteil der online erworbenen rezeptpflichtigen Medikamente - ein zukunftsträchtiges Beispiel für E-Commerce - noch relativ gering ist (Baker 1996), wer- 
den Protokolle entwickelt, um die elektronische Übermittlung von Rezepten einfacher und sicherer zu gestalten. Dadurch könnten Missverständnisse zwischen Arzt und Apotheker minimiert werden. Die Medikamente können in Online-Apotheken zu günstigen Tarifen bestellt und dem Patienten direkt nach Hause geliefert werden (Kacher et al. 2000).

Der vierte Anwendungsbereich - und dieses war Thema der vorliegenden Arbeit sind neue Kommunikationssysteme auf dem Gebiet der Patientenbetreuung und -informationen: Es entwickeln sich zunehmend Internet-basierende Selbsthilfegruppen und Ärzte-/Expertensysteme, die speziell an den Bedarf von Patienten angepasst sind. Dabei geht es neben dem Informationsaustausch oft auch um die emotionale Unterstützung (Kassirer 2000).

Derzeit arbeiten die Online-Ärzte noch unentgeltlich. Bei steigendem Bedarf muss aber über eine Finanzierung dieses Services nachgedacht werden. In Deutschland scheitert die flächendeckende Anwendung von Telemedizin bzw. Telekonsultation noch an der Gesetzgebung und der Berufsordnung der Ärzte, die Ferndiagnosen und -behandlungen gemäß §7 III MBO-Ä 1997 verbietet. Deshalb liegt der Schwerpunkt des Expertenforums bzw. der Telemedizin (momentan) in der Aufklärung und Prävention, da diese Tätigkeit der Ärzte mit der geltenden Berufsordnung grundsätzlich vereinbar ist.

Ähnlich der hier vorgelegten Analyse könnten Anfragen an Websites und Expertenforen auch als Indikator und Gradmesser für die Qualität und Defizite der ambulanten und stationären medizinischen Versorgung herangezogen werden. Das setzt Aufgeschlossenheit und Interesse bei behandelnden Ärzten voraus - und Verzicht auf Überlegenheitsgefühl bei Internet-Ärzten.

Neben den Chancen des erleichterten Informationszugangs entstehen aber auch Risiken: Die im Netz gespeicherten Informationen unterliegen keiner redaktionellen Kontrolle und in vielen Fällen sind die Kompetenz und Objektivität der Anbieter medizinischer Informationen nicht klar. Ansätze zur Qualitätssicherung wird mit Hilfe von "Gütesiegeln" unabhängiger Organisationen wie z. B. das EU-Projekt med-CERTAIN erarbeitet. Die Idee der Bewertung stellt sich jedoch durch die Dynamik des ständig 
wechselnden Internetangebots und die Masse an Informationen als schwierig dar (Eysenbach und Diepgen 1999 b).

Trotz der (noch) bestehenden Risiken in der Telemedizin werden Onlineberatungsservices, wie das Expertenforum, einen wesentlichen Beitrag zur Optimierung von Kommunikationsprozessen im Gesundheitswesen leisten. Viele (potentielle) Patienten haben sich bereits auf den Weg gemacht und die Vorteile solcher Angebote erkannt. Eine genauere Bilanzierung dieser Vorteile (im Sinne von health outcomes) und eine Überprüfung der Ergebnisse dieser Arbeit durch Analyse weiterer InternetExpertenforen bleibt zukünftigen Studien vorbehalten. 


\section{Zusammenfassung}

Hintergrund: Das Internet gewinnt als integraler Bestandteil der medizinischen Versorgung an Bedeutung. Für (potentielle) Patienten, die gezielte Informationen und/oder Entscheidungen wünschen, könnten Expertenforen im Internet als OnlineBeratung durch qualifizierte Fachärzte eine neuartige Form der medizinischen Betreuung sein.

Ziel: Am Beispiel des Expertenforums „rund-ums-baby.de" sollten Informationsbedarf und Nutzungserfahrungen ungewollt kinderloser Paare ermittelt werden.

Methode: (1) Retrospektive Auswertung der bisherigen Anfragen an das Expertenforum „Kinderwunsch“ der Website www.rund-ums-baby.de; (2) Online-Befragung der Nutzer des Expertenforums; (3) Verknüpfung der Anfragen der Nutzer mit ihren Antworten auf die Online-Befragung in Form von Einzelfallanalysen.

Ergebnisse: Es wurden 523 Personen befragt (Antwortrate: 84,4\%); fast ausschließlich Frauen (99,2\%) und - im Vergleich zum Bundesdurchschnitt - häufiger Personen mit höherem Bildungsabschluss. Fast 15\% der Befragten wandten sich wegen ihrer Kinderlosigkeit als erstes an das Expertenforum. Damit war das Expertenforum der zweithäufigste Ansprechpartner nach dem Frauenarzt (75\%) auf der Suche nach Hilfe bei unerfülltem Kinderwunsch. Der Hausarzt spielte als Berater bzw. erster Ansprechpartner bei Kinderlosigkeit mit ca. $2 \%$ eine geringe Rolle. Die inhaltsanalytisch ausgewerteten Anfragen an das Expertenforum ( $N=827)$ ließen sich am häufigsten folgenden Hauptkategorien zuordnen: Wunsch nach „Aufklärung und Erläuterung“ $(66,3 \%)$ und nach „unabhängiger medizinischer Beratung“ (47,9\%). Die Befragten selbst nannten als Hauptgründe für den Besuch des Forums: Suche nach allgemeinen Informationen zur Kinderlosigkeit $(\mathrm{N}=368)$, Fragen zur derzeitigen Therapie $(\mathrm{N}=228)$ und Suche nach fachkompetenter Beratung $\mathrm{zu}$ verschiedenen Behandlungsmethoden ( $N=162)$. Die Mehrzahl der Besucher des Forums $(56,4 \%)$ war mit der Arbeit der Experten zufrieden. Soweit aus den Anfragen bzw. der Befragung Kritik an der derzeitigen ambulanten oder stationären medizinischen Versorgung zum Ausdruck kam, bezog sie sich häufig auf die fehlende Zeit bei Behandlungsterminen und mangeInde Information und Aufklärung durch die Ärzte. Befragte, die die Antworten des Kinderwunsch-Expertenforums mit ihrem behandelnden Arzt besprachen, waren etwas häufiger mit der jeweiligen ärztlichen Betreuung zufrieden als Patienten, die dies nicht taten ( $80,1 \%$ vs. $64,2 \%)$. 
Schlussfolgerung: Die Aussagekraft der Untersuchung wird durch die nicht bevölkerungsrepräsentative Stichprobe limitiert. Konkreter Hilfebedarf besteht hinsichtlich besserer Aufklärung und Beratung bei kinderlosen Paaren. Die Besucher profitieren von den Antworten der Experten und bewerteten das Expertenforum als zuverlässige Quelle für medizinische Informationen - hier speziell für das Thema Kinderlosigkeit. Ärzte sollten daher der Informationssuche ihrer Patienten und auch dem Wunsch nach einer zweiten Meinung in schwierigen Situationen positiv gegenüberstehen und ein offenes Gespräch darüber fördern. Dann könnten Internetangebote, wie das Expertenforum, in Zukunft die Betreuung dieser Patienten/ Paare effektiv unterstützen. 


\section{Anhang}

\subsection{Fragebogen}

\subsubsection{Einleitungstext}

Liebe Besucherin, lieber Besucher des Expertenforums,

die Abteilung Allgemeinmedizin und die Frauenklinik der Universität Göttingen planen eine Studie über Probleme und Informationswünsche von Frauen und Männern mit unerfülltem Kinderwunsch.

Wir bitten Sie, den nachfolgenden Fragebogen zu beantworten. Ihre Antworten werden von uns streng vertraulich behandelt. Ob Sie den Fragebogen ausfüllen oder nicht: Sie erhalten in jedem Fall eine Antwort auf Ihre Fragen. Aber bitte bedenken Sie, dass Ihnen und anderen Betroffenen die Ergebnisse unserer Untersuchung zugute kommen können.

Wenn Sie mehr über uns wissen oder uns etwas mitteilen möchten, dann finden Sie hier weitere Informationen über die Verantwortlichen dieser Studie sowie über das Projekt Kinderwunsch der Abteilung Allgemeinmedizin und die Fertilitätssprechstunde der Frauenklinik der Universität Göttingen.

Herzlichen Dank für Ihr Interesse!

\subsubsection{Filterfrage}

Haben Sie den Fragebogen schon einmal ausgefüllt?

$\begin{array}{ll}\text { Ja } & \begin{array}{l}\text { [automatische Rückleitung zum Forum] } \\ \text { [automatische Weiterleitung zur nächsten } \\ \text { Frage] }\end{array} \\ \begin{array}{l}\text { Ich habe keinen } \\ \text { unerfüllten Kinderwunsch }\end{array} & \text { [automatische Rückleitung zum Forum] }\end{array}$

Würden Sie an der Studie teilnehmen und den Fragebogen ausfüllen?

$\mathrm{Ja}$

Nein [automatische Weiterleitung zum Fragebogen]

[automatische Rückleitung zum Forum] 


\subsubsection{Online-Fragebogen}

1. Wer hat Sie auf dieses Expertenforum aufmerksam gemacht?
a) Arzt/Ärztin
b) Krankenschwester/Pfleger/Arzthelferin
c) Bekannte/Verwandte/Ehemann
d) Zeitschriften/ Fernsehen
e) Durch Zufall entdeckt
f) Systematisch im Netz gesucht
g) Sonstiges:

2. Aus welchem Grund besuchen Sie das Expertenforum? (Mehrfachnennung möglich)
a) Ich suche Informationen, weil sich mein Kinderwunsch noch nicht erfüllt hat
b) Ich habe Fragen zu den Ursachen der Kinderlosigkeit
c) Ich habe Fragen zu den verschiedenen Behandlungen
d) Ich habe Fragen zu meiner derzeitigen Behandlung
e) Ich habe Fragen zu Untersuchungsergebnissen
f) Sonstiges:

3. Stellen Sie heute zum ersten Mal eine Frage an das Expertenforum?

$\mathrm{Ja}$

Nein [automatische Weiterleitung zu Frage 8] [automatische Weiterleitung zu Frage 4]

4. Konnte das Expertenforum Ihre Informationswünsche befriedigen?
a) $\mathrm{Ja}$
b) Nein
c) Teils/teils

Kritik/Lob und Wünsche an das Expertenforum: 
5. Besprechen Sie die Antworten der Experten mit Ihrem Arzt/Ärztin?

$\mathrm{Ja}$

Nein

6. Haben die Antworten der Experten Sie veranlasst, zusätzlich einen anderen Arzt/Ärztin oder Spezialklinik aufzusuchen?

$\mathrm{Ja}$

Nein

7. Haben Sie aufgrund des Ratschlags eines Experten eine Behandlung begonnen?

$\mathrm{Ja}$

Nein

8. Wie lange beschäftigt Sie schon das Problem „Kinderlosigkeit“?
a) ca. _Monate
b) ca. _Jahre

9. Wo suchten Sie zuerst Rat über Ihre Kinderlosigkeit?
a) Bei diesem Expertenforum
b) Hausarzt/ärztin
c) Frauenarzt/ärztin
d) Urologen/in
e) Bekannte/Verwandte
f) Geistlichen, Seelsorger
g) Psychotherapeut/in
h) Heilpraktiker/in
i) Sonstige

10. Sind Sie in Behandlung?

$\mathrm{Ja}$

Nein
Wenn Ja, wie lange: a) seit _ Monaten

b) seit__Jahren 
11. Wie werden Sie zurzeit behandelt? (Mehrfachnennungen möglich)
a) Hormone
b) Künstliche Befruchtung (Beispiel IVF, ICSI, IUI)
c) Psychotherapie
d) Alternative Methoden (z. B. Akupunktur, Homöopathie)
e) Operation
f) Beratung
g) Sonstiges

12. Wie ist die Betreuung durch Ihren Arzt/Ärztin oder Therapeuten/in während Ihrer Behandlung? (Bewerten Sie bitte die Ärzte/Therapeuten, mit denen Sie in Kontakt stehen.)

\section{Betreuung}

Sehr gut Gut Eher schlecht Sehr schlecht
a) Frauenarzt/ärztin
b) Hausarzt/ärztin
c) Arzt/Ärztin in einer Kinderwunschklinik

d) Psychotherapeut/in

O

O
0
O

O

O

e) Androloge/in

0
0
0
0
0

0

0

O

O

O

0

f) Heilpraktiker/in

0

O

O

g) Sonstige

13. Wie fühlen Sie sich durch Ihren Arzt/Ärztin oder Therapeuten/in informiert und aufgeklärt? (Bewerten Sie bitte die Ärzte/Therapeuten, mit denen Sie in Kontakt stehen.)

\section{Informationen/ Aufklärung}

Sehrgut Gut Eher schlecht Sehr schlecht
a) Frauenarzt/ärztin
b) Hausarzt/ärztin
c) Arzt/Ärztin in einer Kinderwunschklinik d) Psychotherapeut/in
O

O

O

O

O

○

o

O
$O$
o

O

O

O 

e) Androloge/in
O
O
$\mathrm{O}$
O
f) Heilpraktiker/in
0
O
0
O
g) Sonstige
0
0
O
O

Ihre Wünsche an den Arzt:

14. Können Sie mit Ihrem behandelnden Arzt/Ärztin oder Therapeuten/in über alle Probleme reden, die Sie bei der Behandlung haben? (Bewerten Sie bitte die Ärzte/Therapeuten, mit denen Sie in Kontakt stehen.)

\begin{tabular}{|c|c|c|c|c|}
\hline & $\mathrm{Ja}$ & Zumeist & Eher selten & Nein \\
\hline a) Frauenarzt/ärztin & O & O & 0 & O \\
\hline b) Hausarzt/ärztin & O & O & 0 & O \\
\hline \multicolumn{5}{|l|}{ c) Arzt/Ärztin in einer } \\
\hline Kinderwunschklinik & O & O & O & $\mathrm{O}$ \\
\hline d) Psychotherapeut/in & O & O & O & O \\
\hline e) Androloge/in & O & $\mathrm{O}$ & 0 & O \\
\hline f) Heilpraktiker/in & O & O & O & O \\
\hline g) Sonstige & 0 & $\mathrm{O}$ & O & $\mathrm{O}$ \\
\hline
\end{tabular}

Wenn „Eher selten“ oder „Nein“, über welche Probleme können Sie mit Ihrem Arzt/Ärztin nicht sprechen ? (Mehrfachantworten möglich)
a) Psychologische Probleme (z.B. Ängste, Depressionen)
b) Partnerschaftsprobleme
c) Sexualität
d) Körperliche Beschwerden (z.B. Schmerzen)
e) Sonstige

15. Wird während Ihrer Behandlung eine psychologische Betreuung angeboten? Ja

Nein

Zum Abschluss bitten wir Sie noch um einige Angaben zu Ihrer Person:

16. Ihr Alter: 
17. Ihr Geschlecht: weiblich

männlich

18. Familienstand:
a) Verheiratet
b) In einer festen Partnerschaft lebend
c) Single
d) Sonstiges

19. Haben Sie leibliche Kinder?

$\mathrm{Ja}$

Nein

20. Schulabschluss:
a) Keinen
b) Hauptschulabschluss
c) Realschulabschluss
d) Abitur
e) Fachhoch/Hochschulabschluss

21. Lage und Größe Ihres Wohnorts:
a) Ländlich strukturiert
b) Kleinstadt
c) Mittelstadt
d) Großstadt

22. In welchem Bundesland bzw. Staat leben Sie?

\section{Zusatzfrage während der Pilotphase:}

23. Hatten Sie Probleme beim Ausfüllen des Fragebogens? 


\subsection{Grundauszählung:}

Filterfrage $A^{1}$ :

Haben Sie den Fragebogen schon ein-

Anzahl

Prozent

mal ausgefüllt?

\begin{tabular}{lrc}
\hline - Ja & 632 & 48,4 \\
- Nein & 620 & 47,5 \\
- Ich habe keinen Kinderwunsch. & 53 & 4,1 \\
\hline Gesamt & 1305 & 100,0 \\
\hline
\end{tabular}

Filterfrage B:

Würden Sie an der Studie teilnehmen

Anzahl

Prozent

und den Fragebogen ausfüllen?

\begin{tabular}{lcc}
\hline - Ja & 523 & 84,4 \\
- Nein & 97 & 15,6 \\
\hline Gesamt & 620 & 100,0 \\
\hline
\end{tabular}

\section{Frage 1:}

Wer hat Sie auf das Expertenforum $\quad$ Anzahl $\quad$ Prozent aufmerksam gemacht?

\begin{tabular}{lrr}
\hline - Arzt & 6 & 1,3 \\
- Pflegepersonal & 0 & 0,0 \\
- Angehörige, Bekannte & 48 & 10,1 \\
- Zeitschriften, Fernsehen & 17 & 3,6 \\
- Durch Zufall entdeckt & 220 & 46,4 \\
- Systematisch im Netz gesucht & 183 & 38,6 \\
\hline Gesamt & 474 & 100,0
\end{tabular}

Missings: 49

\footnotetext{
${ }^{1}$ Die Nummerierung erfolgt analog zum Fragebogen. Die Antworten auf die offenen Fragen werden an dieser Stelle nicht aufgeführt.
} 


\section{Frage 2:}

Aus welchem Grund besuchen Sie das

Anzahl

Prozent

Expertenforum? (Mehrfachantworten

möglich)

\begin{tabular}{lrc}
\hline - Allgemeine Informationen & 368 & 72,9 \\
- Fragen zur derzeitigen Behandlung & 228 & 45,1 \\
- Fragen zu Behandlungsmethoden & 162 & 32,1 \\
- Fragen zu den Ursachen der Kinder- & 128 & 25,5 \\
$\quad$ losigkeit & & \\
- Fragen zu Untersuchungsergebnissen & 111 & 22,0 \\
- Sonstige & 39 & 7,7 \\
\hline Gesamt & 505 &
\end{tabular}

Missings: 18

\section{Frage 3:}

Stellen Sie heute zum ersten Mal eine

Frage an das Expertenforum?

\begin{tabular}{lcc}
\hline - Ja & 276 & 55,1 \\
- Nein & 225 & 44,9 \\
\hline Gesamt & 501 & 100,0
\end{tabular}

Missings: 22

Frage 4: (nur Befragte, die mehrmals das Expertenforum besuchten)

Konnte das Expertenforum Ihre InforAnzahl

Prozent

mationswünsche befriedigen?

\begin{tabular}{lrr}
\hline - Ja & 123 & 56,4 \\
- Nein & 7 & 3,2 \\
- Teils/ teils & 88 & 40,4 \\
\hline Gesamt & 218 & 100,0
\end{tabular}

Missings: 29

Frage 5: (nur Befragte, die mehrmals das Expertenforum besuchten)

Besprechen Sie die Antworten der Ex- Anzahl Prozent

perten mit Ihrem Arzt?

\begin{tabular}{lcc}
\hline - Ja & 105 & 47,1 \\
- Nein & 118 & 52,9 \\
\hline Gesamt & 223 & 100,0 \\
\hline
\end{tabular}


Frage 6: (nur Befragte, die mehrmals das Expertenforum besuchten)

Haben die Antworten der Experten Sie

Anzahl

Prozent

veranlasst, zusätzlich einen anderen

Arzt oder Spezialklinik aufzusuchen?

\begin{tabular}{lrc}
\hline - Ja & 51 & 23,1 \\
- Nein & 170 & 76,9 \\
\hline Gesamt & 221 & 100,0 \\
\hline
\end{tabular}

Missings: 27

Frage 7: (nur Befragte, die mehrmals das Expertenforum besuchten)

Haben Sie aufgrund des Ratschlags Anzahl Prozent

eines Experten eine Behandlung be-

gonnen?

\begin{tabular}{lrc}
\hline - Ja & 56 & 25,2 \\
- Nein & 166 & 74,8 \\
\hline Gesamt & 222 & 100,0 \\
\hline
\end{tabular}

Missings: 26

\section{Frage 8:}

\begin{tabular}{lcccc}
\hline $\begin{array}{l}\text { Wie lange beschäftigt Sie das Problem } \\
\text { „Kinderlosigkeit“? }\end{array}$ & Range & Mean & Median & $\begin{array}{c}\text { Std Devia- } \\
\text { tion }\end{array}$ \\
\hline - in Monaten & $0-240$ & 28 & 18 & 29,8
\end{tabular}

\section{Frage 9:}

Wo suchten Sie zuerst Rat über Ihre

Anzahl Prozent

Kinderlosigkeit?

\begin{tabular}{lrr}
\hline - Expertenforum & 72 & 14,9 \\
- Hausarzt & 8 & 1,6 \\
- Frauenarzt & 361 & 74,6 \\
- Urologe & 9 & 1,9 \\
- Angehörige, Bekannte & 26 & 5,4 \\
- Geistlicher & 2 & 0,4 \\
- Psychotherapeut & 2 & 0,4 \\
- Heilpraktiker & 4 & 0,8 \\
\hline Gesamt & 484 & 100,0
\end{tabular}

Missings: 39 
Frage 10 a:

\begin{tabular}{lcc}
\hline Sind Sie in Behandlung? & Anzahl & Prozent \\
\hline - Ja & 319 & 64,6 \\
- Nein & 175 & 35,4 \\
\hline Gesamt & 494 & 100,0 \\
\hline
\end{tabular}

Missings: 29

Frage 10 b: (nur Befragte, die in Behandlung waren)

\begin{tabular}{llccc}
\hline Wie lang sind Sie in Behandlung? & Range & Mean & Median & $\begin{array}{c}\text { Std Devia- } \\
\text { tion }\end{array}$ \\
\hline - in Monaten & $0-239,5$ & 18,4 & 10,0 & 26,5 \\
\hline
\end{tabular}

Frage 11: (nur Befragte, die in Behandlung waren)

Wie werden Sie zurzeit behandelt? Anzahl

Prozent

(Mehrfachantworten möglich)

- Hormone 146 45,8

- Künstliche Befruchtung (IVF, ICSI) 144

45,1

- Psychotherapie

- Alternative Methoden

- Operation

- Beratung

Gesamt

320

Missings: 28

Frage 12: (nur Befragte, die in Behandlung waren)

\begin{tabular}{l|rc|rc|cc}
\hline $\begin{array}{l}\text { Wie ist die Betreuung } \\
\text { Ihres Arztes oder The- } \\
\text { rapeuten während Ih- }\end{array}$ & $\begin{array}{r}\text { Befragte mit Frau- } \\
\text { enarzt }\end{array}$ & $\begin{array}{r}\text { Befragte mit Haus- } \\
\text { arzt }\end{array}$ & $\begin{array}{l}\text { Befragte mit Arzt im } \\
\text { reproduktionsmedi- } \\
\text { zinischen Zentrum }\end{array}$ \\
\hline & Anzahl & Prozent & Anzahl & Prozent & Anzahl & Prozent \\
- Sehr gut & 103 & 35,8 & 18 & 21,2 & 70 & 42,4 \\
- Gut & 127 & 44,1 & 38 & 44,7 & 71 & 43,1 \\
- eher schlecht & 43 & 14,9 & 17 & 20,0 & 17 & 10,3 \\
- schlecht & 15 & 5,2 & 12 & 14,1 & 7 & 4,2 \\
\hline Gesamt & 288 & 100,0 & 85 & 100,0 & 165 & 100,0 \\
\hline
\end{tabular}


Frage 13: (nur Befragte, die in Behandlung waren)

\begin{tabular}{l|rc|rc|cc}
\hline $\begin{array}{l}\text { Wie fühlen Sie sich } \\
\text { durch Ihren Arzt infor- } \\
\text { miert und aufgeklärt? }\end{array}$ & $\begin{array}{c}\text { Befragte mit Frau- } \\
\text { enarzt }\end{array}$ & $\begin{array}{c}\text { Befragte mit Haus- } \\
\text { arzt }\end{array}$ & $\begin{array}{l}\text { Befragte mit Arzt im } \\
\text { reproduktionsmedi- } \\
\text { zinischen Zentrum }\end{array}$ \\
\hline & Anzahl & Prozent & Anzahl & Prozent & Anzahl & Prozent \\
- Sehr gut & 79 & 27,6 & 11 & 15,1 & 59 & 36,2 \\
- Gut & 114 & 39,7 & 32 & 43,8 & 62 & 38,0 \\
- eher schlecht & 73 & 25,5 & 18 & 24,7 & 36 & 22,1 \\
- schlecht & 21 & 7,3 & 12 & 16,4 & 6 & 3,7 \\
\hline Gesamt & 287 & 100,0 & 73 & 100,0 & 163 & 100,0 \\
\hline
\end{tabular}

Frage 14 a: (nur Befragte, die in Behandlung waren)

\begin{tabular}{l|rc|rc|cc}
\hline $\begin{array}{l}\text { Können Sie mit Ihrem } \\
\text { behandelnden Arzt } \\
\text { über alle Probleme } \\
\text { reden, die Sie bei der } \\
\text { Behandlung haben? }\end{array}$ & $\begin{array}{c}\text { Befragte mit Frau- } \\
\text { enarzt }\end{array}$ & $\begin{array}{c}\text { Befragte mit Haus- } \\
\text { arzt }\end{array}$ & $\begin{array}{l}\text { Befragte mit Arzt im } \\
\text { reproduktionsmedi- } \\
\text { zinischen Zentrum }\end{array}$ \\
\hline & Anzahl & Prozent & Anzahl & Prozent & Anzahl & Prozent \\
- Ja & 121 & 42,5 & 31 & 38,3 & 66 & 40,5 \\
- Zumeist & 99 & 34,7 & 21 & 26,0 & 48 & 29,4 \\
- Eher selten & 49 & 17,2 & 17 & 20,9 & 38 & 23,3 \\
- Nein & 16 & 5,6 & 12 & 14,8 & 11 & 6,8 \\
\hline Gesamt & 285 & 100,0 & 81 & 100,0 & 163 & 100,0 \\
\hline
\end{tabular}

Frage 14 b: (nur Befragte, die eher selten oder nicht mit Ihrem Arzt über Ihre Probleme reden konnten)

\begin{tabular}{lcc}
\hline $\begin{array}{l}\text { Über welche Probleme können Sie mit } \\
\text { Ihrem Arzt nicht sprechen? }\end{array}$ & Anzahl & Prozent \\
$\begin{array}{l}\text { (Mehrfachantworten möglich) } \\
\text { - Psychologische Probleme }\end{array}$ & 79 & \\
- Probleme in der Partnerschaft & 30 & 55,2 \\
- Sexualität & 37 & 21,0 \\
- Körperliche Beschwerden & 30 & 21,0 \\
\hline Gesamt & 176 & \\
\hline
\end{tabular}


Frage 15: (nur Befragte, die in Behandlung waren)

Wird während Ihrer Behandlung eine Anzahl

Prozent

psychologische Betreuung angeboten?

\begin{tabular}{lrr}
\hline - Ja & 62 & 19,8 \\
- Nein & 251 & 80,2 \\
\hline Gesamt & 313 & 100,0
\end{tabular}

Missings: 6

Frage 16:

Wie alt sind Sie?

Range Mean Median Std Devia-

tion

- in Jahren

$18-43$

29,2

29

4,9

\section{Frage 17:}

\begin{tabular}{lcc}
\hline Ihr Geschlecht? & Anzahl & Prozent \\
\hline - Weiblich & 496 & 99,2 \\
- Männlich & 4 & 0,8 \\
\hline Gesamt & 500 & 100,0 \\
\hline
\end{tabular}

Missings: 23

\section{Frage 18:}

\begin{tabular}{lcc}
\hline Ihr Familienstand? & Anzahl & Prozent \\
\hline - Verheiratet & 364 & 72,5 \\
- In fester Partnerschaft lebend & 134 & 26,7 \\
- Single & 4 & 0,8 \\
\hline Gesamt & 502 & 100,0
\end{tabular}

Missings:21

Frage 19:

\begin{tabular}{lcc}
\hline Haben Sie leibliche Kinder? & Anzahl & Prozent \\
\hline - Ja & 180 & 35,8 \\
- Nein & 323 & 64,2 \\
\hline Gesamt & 503 & 100,0
\end{tabular}

Missings: 20 
Frage 20:

Ihr Schulabschluss?

Anzahl

Prozent

- Keinen

2

0,4

- Hauptschulabschluss

60

11,9

- Realschulabschluss

206

40,9

- Abitur

99

19,6

- Fachhoch/Hochschulabschluss

137

27,2

Gesamt

504 100,0

Missings: 19

Frage 21:

\begin{tabular}{lcc}
\hline Wie groß ist Ihre Stadt? & Anzahl & Prozent \\
\hline - ländlich strukturiert & 153 & 30,5 \\
- Kleinstadt & 102 & 20,4 \\
- Mittelstadt & 112 & 22,4 \\
- Großstadt & 134 & 26,7 \\
\hline Gesamt & 501 & 100,0
\end{tabular}

Missings: 22 


\section{Literaturverzeichnis}

Abbey A, Halman LJ (1995): The role of perceived control, attributions and meaning in members of infertile couple well-being. J Soc Clin Psychol, 14, 271-296

Abbey A, Andrews FM, Halman LJ (1991): Gender's role in responses to infertility. Psychol Women Q, 14, 295-316

ADM: Arbeitskreis Deutscher Markt- und Sozialforschung, Arbeitsgemeinschaft Sozialwissenschaftlicher Institute (ASI), Berufsverband Deutscher Markt- und Sozialforscher (BVM), Deutsche Gesellschaft für Online-Forschung (D.G.O.F.): Standards zur Qualitätssicherung für Online-Befragungen. ADM, Frankfurt 2001

Anderson JG, Rainey MR, Eysenbach G (2003): The impact of cyberhealthcare on the physician-patient relationship. J Med Syst, 27, 67-84

Andrews FM, Abbey A, Halman LJ (1991): Stress from infertility, marriage factors, and subjective well-being of wives and husbands. J Health Soc Behav, $\underline{32}, 238-253$

Bader SA, Braude RM (1998): "Patient informatics": Creating new partnerships in medical decision making. Acad Med, $\underline{73}, 408-411$

BÄK, Bundesärztekammer (1997): Musterberufsordnung für Ärzte [MBO], §7, Par. 3 www.bundesaerztekammer.de/30/Berufsordnung/10Mbo/index.html

Baker R (1996): Nobody's talking. Mark Res, $\underline{8}, 22-24$

Bandilla W, Bosnjak M, Altdorfer P (2001): Effekte des Erhebungsverfahrens? Ein Vergleich zwischen einer web-basierten und einer schriftlichen Befragung zum ISSP-Modul Umwelt. ZUMA-Nachrichten, $\underline{49}$, 7-28

Batinic B, Bosnjak M: Fragebogen-Untersuchungen im Internet; in: Internet für Psychologen; hrsg. v. Batinic B, Bosnjak M; Hofgrefe, Göttingen 1997, 221-243

Baur C (2000): Limiting factors on the transformative powers e-mail in patient-physician relationship: a critical analysis. Health Commun, 12, 239-259

BCG, Boston Consulting Group: Patients and the internet. 2001

www.bcg.com/publications/files/Patients_and_the_internet_Jan_01_summary.pdf (zugegriffen am 05/01/2004)

Bengel J, Carl C, Mild U, Strauß B (2000): Langfristige Folgen von Kinderlosigkeit: Eine Übersicht. Z Klin Psychol Psychother, 29, 3-15

Berg B: Qualitative research methods for social science. Allyn und Bacon, Boston 1989

Berg BJ, Wilson JF, Weingartner PJ (1991): Psychological sequelae of infertility treatment: The role of gender and sex-role identification. Soc Sci Med, $\underline{33}, 1071-1080$

Bernt H, Bernt WD, Tacke S (1992): Sterilität - Frauensache? Bewältigungsverhalten und Paarstruktur von sterilen Paaren verschiedener Diagnosegruppen. Psychother Psychosom Med Psychol, 느, 236-241

Boeger A, Gnatzy A, Sahlmann M (1999): Bewältigungsreaktionen nach erfolgloser Sterilitätsbehandlung. Rep Psychol, $\underline{5}$, 367-375 
Bortz J, Döring N: Forschungsmethoden und Evaluationen; Springer, Berlin, Heidelberg 2002

Brähler E, Stöbel-Richter Y (2002): Zum Wandel im Reproduktionsverhalten in Deutschland und im europäischen Vergleich. Z Arztl Fortbild Qualitatssich, 96, 459-467

Brauchli K, Helfrich M, Christen H, Jundt G (2002): Die Zukunft der Telepathologie - Ein im Internet „verteiltes System“ mit „offenen Standards“. Pathologe, 23, 198-206

Callan V, Hennessey J (1989): Strategies of coping with infertility. Br J Med Psychol, $\underline{62}$, 343-354

Charnock D: Das DISCERN-Handbuch. Qualitätskriterien für Patienteninformation über Behandlungsalternativen. Zuckschwerdt, München 2000

Cyberdialogue: Impacts of the internet on the doctor-patient relationship. 2000 www.cyberdialogue.com (zugegriffen am 06/12/2002)

Denzin NK: The research act. N.J.: Prentice Hall, Englewood Cliffs 1989, 237-241

Diaz JA, Griffith RA, James JN (2002): Patient's use of the internet for medical information. J Gen Intern Med, 17, 180-185

ECIN: Electronic Commerce Info Net (2002):

www.ecin.de/news/2001/02/08/01528/print.html (zugegriffen am 12/06/2002)

Emnid (2002): Die deutsche Internet-Teilung. www.emnid.tnsofres.com/presse/p2002_06_04.html (zugegriffen am 12/06/2002)

EORG: European Opinion Research Group (2003): European Union citizens and sources of information about health.; www.eorg.com (zugegriffen am 04/12/2003)

Epstein YM, Rosenberg HS, Venet Grant T (2002): Use of the internet as the only outlet for talking about infertility. Fertil Steril, $\underline{78}, 507-514$

Expertenforum „Kinderwunsch“, www.rund-ums-baby.de/kinderwunsch_expertenforum/ mebboard.php3

Eysenbach G (2002): Issues in evaluating health websites in an internet-based randomized controlled trial. J Med Internet Res, 4 (3):e17, www.jmir.org/2002/3/e17 (zugegriffen am 01/06/2003)

Eysenbach G, Diepgen TL (1999 a): Patients looking for information on the internet and seeking teleadvice: motivation, expectations, and misconceptions as expressed in E-mails sent to physicians. Arch Dermatol, $\underline{135}, 151-156$

Eysenbach G, Diepgen TL (1999 b): Das Internet: Bedeutung für Prävention, Gesundheitsförderung und evidenzbasierter Medizin. Dtsch Med Wochenschr, 느, 1404-1405

Felder H, Pantke-Ehlers E, Meyer F: Subjektive Körperbeschwerden von Frauen und Männern während einer In-vitro-Fertilisationsbehandlung; in: Fruchtbarkeitsstörungen (Jahrbuch der medizinischen Psychologie, Band 17); hrsg. v. Brähler E, Felder H, Strauß B; Hofgrefe, Göttingen 2000, 102-123

Fischl FH: Begriffsbestimmung und Pathogenese: Sterilität - Fertilität; in: Kinderwunsch Möglichkeiten, Erfüllbarkeit, und Machbarkeit im neuen Jahrtausend; hrsg. v. Fischl FH; Krause und Pachernegg - Verlag für Medizin und Wissenschaft, Gablitz 2000, 47- 55 
Flick U: Triangulation; in: Handbuch qualitative Sozialforschung: Grundlagen, Konzepte, Methoden und Anwendungen; hrsg. v. Flick U, v. Kardorff E, Keupp H, v. Rosenstiel L, Wolff S, Beltz, Weinheim 1995, 432-434

Forrester Research (2002): Why doctors hate the net.

www.forrester.com/ER/research/interview/0,1338,9114,FF.html (zugegriffen am 20/12/ 2003)

Freeman EW, Rickels K, Tausig J (1987): Emotional and psychological factors in follow-up of women after IVF-ET treatment: a pilot investigation. Acta Obstet Gynecol Scand, $\underline{66}, 517-521$

Frey JH, Kunz G, Lüschen G: Telefonumfragen in der Sozialforschung. Westdeutscher Verlag, Opladen 1990

Fuchs M: Umfrageforschung mit Telefon und Computer: Eine Einführung in die computergestützte telefonische Befragung. Beltz Psychologie VerlagsUnion, Weinheim 1994, 129

Fuchslocher H, Fritz M: Computergestützte Marktforschung - Erfahrungen aus dem Bereich Messemarktforschung; in: Handbuch des Electronic Marketing; hrsg. v. Hermanns A, Flegel V; Beck, München 1992, 217-230

Gerber B, Eiser A (2001): The patient-physician relationship in the internet age: Future prospects and research agenda. J Med Internet Res, $\underline{3}$, e15 (zugegriffen am 01/06/2003)

Google: Google offers immediate access to 3 billion Web documents [press release]. Mountain View, Calif: Google Inc; 2001 Dec 11.

www.google.com/press/ pressrel/3billion.html (zugegriffen am 01/09/2003)

Greil AL, Leitko TA, Porter KL (1988): Infertility: his and hers. Gend Soc, 2, 172- 199

Guttormsen G (1992): Unfreiwillige Kinderlosigkeit: Ein Familienproblem. Prax Kinderpsychol Kinderpsychiatr, $\underline{41}$, 247-252

Hansen M, Kurinczuk JJ, Bower C, Webb S (2002): The risk of major birth defects after intracytoplasmic sperm injection and in vitro fertilization. N Engl J Med, $\underline{346}, 725-730$

Hartfield G: Wörterbuch der Soziologie, Stuttgart 1982, 160

Heinzow B, Hanf V: Umwelt und Sterilität; in: Moderne Fortpflanzungsmedizin - Grundlagen, IVF, ethische und juristische Aspekte; hrsg. v. Tinneberg HR, Ottmar C; Thieme, Stuttgart 1995, 78-83

Henning K, Strauß B: Psychological and psychosomatic aspects of involuntary childlessness: State of research at the end of the 1990's; in: Involuntary childlessness - Psychological assessment, counseling and psychotherapy; hrsg. v. Strauß B; Hogrefe und Huber, Seattle 2002, 3-18

HFEA: Human Fertilisation and Embryology Authority: $9^{\text {th }}$ Annual Report \& Accounts 2000; www.hfea.gov.uk/downloads/default.htm (zugegriffen am 01/06/2002)

Himmel W, Ittner E, Kochen MM (1997): Management of involuntary childlessness. Br J Gen Pract, $\underline{47}, 111-118$

Hinney B, Michelmann HW, Göttingen: Fertilitätsstörungen - Was tun, wenn es mit dem Kinderkriegen nicht klappt? unveröffentlichtes Dokument; schriftliche Mitteilung 2001 
Hölzle C: Probleme des unerfüllten Kinderwunsches und seiner medizinischen Behandlung; in: Kinder machen. Strategien der Kontrolle weiblicher Fruchtbarkeit; hrsg. v. Pauritsch G, Frankele B, List E; Wiener Frauenverlag, Wien 1988, 10-21

Houston TK, Cooper LA, Ford DE (2002): Internet support groups for depression: a 1-year prospective cohort study. www.ajp.psychiatryonline.org/cgi/content/full/ 159/12/2062 (zugegriffen am 05/04/2003)

Huinink J, Brähler E: Die Häufigkeit gewollter und ungewollter Kinderlosigkeit; in: Fruchtbarkeitsstörungen (Jahrbuch der Medizinischen Psychologie Band 17); hrsg. v. Brähler E, Felder H, Strauß B; Hofgrefe, Göttingen 2000, 43-55

Ittner E, Himmel W, Kochen M (1997): Gehört das Problem "ungewollte Kinderlosigkeit" zum familienmedizinischen Auftrag des Hausarztes? Psychother Psychosom Med Psychol, 47, $263-270$

Ittner E, Himmel W, Kochen M (1998): Erwartungen hausärztlicher Patienten an eine Betreuung bei ungewollter Kinderlosigkeit. Z Gesundheitspsychol, $\underline{6}$, 37-39

Jakob A: Möglichkeiten und Grenzen der Triangulation quantitativer und qualitativer Daten am Beispiel der (Re-)Konstruktion einer Typologie erwerbsbiographischer Sicherheitskonzepte, Arbeitspapier 2 des SFB 536 Reflexive Modernisierung München, Mai 2001, www.sfb536.mwn.de/arbeitspapiere/ap2-jakob.pdf (zugegriffen am 06/12/2002)

Johnson CL, Catalano DJ (1981): Childless elderly and their family support. Gerontologist, $\underline{21}, 610-618$

Kacher C, Wiest A, Schumacher N (2000): E-Health: Chancen und Risiken für Ärzte, Patienten und Kostenträger. Z Allgemeinmed, $\underline{76}$, 607-613

Kassirer JP (2000): Patients, physicians, and the internet. Health Aff, $\underline{19}, 115-123$

Kedar I, Ternullo JL, Weinrib CE, Kelleher KH, Brandling-Bennett H, Kvedar JC (2003): Internet based consultations to transfer knowledge for patients requiring specialised care: retrospective case view. BMJ, $\underline{326}$, 696-699

Keith PM (1983): A comparison of the resources of parents and childless men and women in very old age. Fam Relat, 32, 403-409

Kentenich H: Psychosomatisch orientierte Beratung und Behandlung steriler Paare; in: Kinderwunsch - Möglichkeiten, Erfüllbarkeit und Machbarkeit im neuen Jahrtausend; hrsg. v. Fischl FH; Krause und Pachernegg - Verlag für Medizin und Wissenschaft, Gablitz 2000, 4246

Kentenich H, Hölzle C, Schiady H, Stauber M (1987): „Am schlimmsten ist das Warten!“ Wie Paare die In-vitro-Fertilisation erleben. Sexualmedizin, 16, 364-370

Kiley R (1999): Editorial - Internet Statistics. Health information on the Internet; 10: 1-2. www.rsm.ac.uk/hii/issue10.pdf (zugegriffen am 05/05/2002)

Kochen MM, Himmel W, Ittner I, Kapmeyer A: Verbesserung der psychosozialen und medinischen Betreuung von Kinderwunschpatienten in der primärärztlichen Versorgung - BMBFFörderungsschwerpunkt „Fertilitätsstörungen“, Technische Informationsbibliothek, Hannover 2001, www.edok01.tib.uni-hannover.de/edoks/e01fb01/330231847I.pdf (zugegriffen am 28/ 07/2003) 
Köhler C, Eysenbach G (2002): Das Internet - Chancen, Risiken und Perspektiven für den chirurgischen Patienten. Chirurg, $\underline{73}, 410-416$

Koubenec HJ (2001): Patienteninformation im Internet. Z Arztl Fortbild Qualitatssich, $\underline{95}$, 297317

Kowalcek I, Wihstutz N, Burhrow G, Diedrich K (2000): Wenn das Wunschkind auf sich warten lässt - Krankheitsverarbeitung ungewollt kinderloser Frauen und Männer. Zentralbl Gynakol, 122, 75-81

Kronz JD, Westra WH, Epstein JI (1999): Mandatory second opinion surgical pathology at a large referral hospital. Cancer, $\underline{86}, 2426-2435$

Küchenhoff J (1999): Der unerfüllte Kinderwunsch - Worunter leiden Männer? Ther Umsch, $\underline{56}, 260-264$

Kuzel A, Like R: Standards of thrustworthiness for qualitative studies in primary care; in: Primary care research, Volume 1; hrsg. v. Norton PG, Stewart M, Tudiver F, Bass MJ, Dunn EV; Sage, Newbury Park 1991, 138-158

Lalos A, Lalos O, Jacobson L, von Schoultz B (1986): Depression, guilt, and isolation among infertile women and their partners. J Psychosomc Obstet Gynaecol, $\underline{5}, 197-206$

Larkins M (2003): New internet brings medicine up to speed. Lancet, $\underline{361}, 844-845$

Leiblum SR, Kemmann E, Lane M (1987): The psychological concomitants of in vitro fertilization. J Psychosom Obstet Gynaecol, $\underline{6}, 165-178$

Ludwig M (2002): Fehlbildungen nach ICSI - Es liegt an den Eltern.

www.aerztlichepraxis.de/db/shownews/1018439193/4/4/news.html (zugegriffen am 14/07/ 2002)

Machin D, Campbell MJ, Fayers PM, Pinol APY: Sample size tables for clinical studies. Blackwell Science, Oxford 1997

Mayring P: Qualitative Inhaltsanalyse. Grundlagen und Techniken. Deutscher Studienverlag, Weinheim 1988, 75

McEwan KL, Costello CG, Taylor PJ (1987): Adjustment to infertility. J Abnorm Psychol, $\underline{96}$, 108-116

Menning B (1980): The emotional need of infertile couples. Fertil Steril 34, 313- 319

Moorman PW, Van der Lei J (1999): An inventory of publications on electronic patient records. Methods Inf Med, $\underline{38}, 294-297$

Muhr T: Atlas.ti - The User's Guide. Scientific Software Development, Berlin 1997

Murero M, D'Ancona G, Karamanoukian H. (2001): Use of the internet by patients before and after cardiac surgery: telephone survey. J Med Internet Res 2001, 3 (3): e27 (zugegriffen am $13 / 05 / 2003)$

Nielsen/NetRatings (2002 a): Nielsen//Netratings reports a record half billion people worldwide now have home internet access. www.nielsen-netratings.com (zugegriffen am 01/06/2002) 
Nielsen/NetRatings (2002 b): Europäische Internetnutzung Januar 2002. www.nielsennetratings.com (zugegriffen am 01/06/2002)

Nieschlag E, Behre HM: Andrologie - Grundlagen und Klinik der reproduktiven Gesundheit des Mannes. Springer, Berlin 1996, 52-54

Nijs P, Koninck PR, Verstraeten D, Mullens A, Nicasy H (1984): Psychological factors of female infertility. Eur J Obstet Gynaecol Reprod Biol, 18, 375-379

Ningel K, Strauß B: Psychological diagnosis, counseling, and psychotherapy in infertility medicine - an overview; in: Involuntary childlessness - Psychological assessment, counseling and psychotherapy; hrsg. v. Strauß B; Hogrefe und Huber, Seattle 2002, 19-34

NUA internet surveys (2000): www.NUAinternetsurveys.com (zugegriffen am 06/12/2000)

O'Connor J, Johanson J (2000): Use of the web for medical information by a gastroenterology clinic population. JAMA, 284, 1962-1964

Onnen-Isemann C (2000): Ungewollte Kinderlosigkeit und die Auswirkungen der Reproduktionsmedizin: Der Fall Deutschland. Forum: Qualitative social research [Online Journal] 1 (1) http://www.qualitative-research.net/fqs (zugegriffen am 05/05/2001)

Patt MR, Houston TK, Jenckes MW, Sands DZ, Ford DE (2003): Doctors who are using email with their patients: a qualitative exploration. J Med Internet Res, 5 (2), e9 (zugegriffen am 06/05/2003)

Pennanen P : Sozialmedizinische und sozialpsychologische Faktoren bei Kinderlosigkeit, Subfekundität und Infertilität und deren Untersuchungen und Behandlungen. Eine epidemiologische Studie. Med. Dissertation, Hamburg 1993

Plas E, Riedl CR: Der männliche Sterilitätsfaktor und seine therapeutischen Möglichkeiten in der assistierten Reproduktionsmedizin; in: Kinderwunsch - Möglichkeiten, Erfüllbarkeit, und Machbarkeit im neuen Jahrtausend; hrsg. v. Fischl FH; Krause und Pachernegg - Verlag für Medizin und Wissenschaft, Gablitz 2000, 123-134

Prein G, Kelle U, Kluge S: Strategien zur Integration quantitativer und qualitativer Auswertungsverfahren. Arbeitspapier 19 des SFB 186, Eigenverlag, Bremen 1993.

Richardson R (2002): eHealth for Europe. Eurohealth, $\underline{8}$, 1-4

Rund ums Baby - Das Online-Magazin für Mamis und Papis - und alle, die es noch werden möchten. www.rund-ums-baby.de

Sänger S (1999): Medizin und Internet in Deutschland. Anaesthesist, $\underline{48}, 751-754$

Scherzer W, Georgopoulos A, Rest E: Patienteninformation im Internet; www.patienteninformation.at/main/text.htm (zugegriffen am 08/10/2001)

Schieve LA, Meikle SF, Ferrre C, Peterson HB, Jeng G, Wilcox LS (2002): Low and very low birth weight in infants conceived with use of assisted reproductive technology. $\mathrm{N}$ Engl J Med, $\underline{346}, 731-737$

Schill WB, Haidl G: Andrologische Grundlagen der Fortpflanzungsmedizin unter Berücksichtigung möglicher Sterilitätsursachen; in: Moderne Fortpflanzungsmedizin - Grundlagen, IVF, ethische und juristische Aspekte; hrsg. v. Tinneberg HR, Ottmar C; Thieme, Stuttgart 1995, 36-42 
Schmidt S, Koch U (2003): Telemedizin aus medizinpsychologischer Perspektive - Der Einfluss von Telematikanwendungen auf die Arzt-Patientbeziehung. Z Med Psychol, 12, 105117

Schnell R: Nonresponse in Bevölkerungsumfragen - Ausmaß, Entwicklung, Ursache. Leske und Budrich, Opladen 1997

Schröter M, Himmel W, Ittner E, Kochen MM (1998): Die Suche nach sozialer Unterstützung bei ungewollter Kinderlosigkeit. Z Gesundheitswiss, $\underline{6}$, 327-340

Schuhrke B (1993): Psychische Probleme bei der medizinischen Unfruchtbarkeitsbehandlung und ihre Bewältigung: Ein Überblick. Verhaltensmodifikation Verhaltensmed, 14, 244270

Silberg W, Lundberg G, Musacchio R (1997): Assessing, controlling and assurring the quality of medical information on the internet. Caveant lector et viewor - Let reader and viewer beware. JAMA, 277, 1244-1245

Stanton AL, Tennen H, Affleck G Mendola R (1991): Cognitive appraisal and adjustment to infertility. Women Health, $\underline{17}$, 1-15

Stanton AL, Tennen H, Affleck G, Mendola R (1992): Coping and adjustment to infertility. J Soc Clin Psychol, 11, 1-13

Statistisches Bundesamt Deutschland (2002): Ausstattung privater Haushalte mit Informationstechnik. www.destatis.de/basis/d/evs/budtab2.htm (zugegriffen am 18/08/2002)

Stauber M (1996) : Ein Kind um jeden Preis? Zur Notwendigkeit von Denkpausen in der Reproduktionsmedizin. Z Allgemeinmed, $\underline{72}, 593$

Stauber M, Kentenich H, Maaßen V, Dincer C, Schmiady H: Psychosomatische Modelle für die extrakorporale Fertilisation; in: Psychosomatische Probleme in der Gynäkologie und Geburtshilfe; hrsg. v. Poethen H, Fervers-Schorre A, Stauber M; Springer, Berlin 1986, 39-51

Strauss AL: Grundlagen qualitativer Sozialforschung - Datenanalyse und Theorienbildung in der empirischen soziologischen Forschung. Fink, München 1991

Strauß B, Argiriou C, Buck S, Mettler L: Die In-vitro-Fertilisation im Rückblick: Subjektives Erleben und psychische Folgen im Urteil betroffener Paare; in: Psychologische Probleme in der Reproduktionsmedizin (Jahrbuch der medizinischen Psychologie, Band 5); hrsg. v. Brähler E, Meyer A; Springer, Berlin, Heidelberg 1991, 89-110

Strauß B, Hepp U, Städing G, Mettler L: Fokale Beratungskonzepte in der Fertilitätsmedizin, in: Fruchtbarkeitsstörungen (Jahrbuch der medizinischen Psychologie, Band 17); hrsg. v. Brähler E, Felder H, Strauß B; Hofgrefe, Göttingen 2000, 274-290

Sudman S, Bradburn N: Response Effects in Surveys. Aline, Chicago 1974

Swoboda W: Medizinische Informationssysteme via Computerwerk: Vademecum der Zukunft? in: Möglichkeiten und Grenzen sowie Datenschutzaspekte bei der Internet-Nutzung in Forschungsprojekten; hrsg. v. Weinkauf B, Lieder V, Kirch W; Eigenverlag Dresden 1997, $95-120$

Taylor H: The Harris Poll \#19: Cyberchondriacs Update. 2001 Apr 18.

www.harrisinteractive.com/harris_poll/index.asp?PID=229 (zugegriffen am 06/06/2002) 
Taylor MRG, Alman A, Manchester DK (2001): Use of the internet by patients and their families to obtain genetics-related informations. Mayo Clin Proc, $\underline{76}, 772-776$

Templeton AA: The epidemiology of infertility; in: Infertility; hrsg. v. Templeton AA, Drife JO; Springer, Heidelberg, Berlin 1992, 134-142

Theobald A: Das World Wide Web als Befragungsinstrument. Reihe: Interaktives Marketing; hrsg. v. Silberer G; Gabler Edition Wissenschaft, Wiesbaden 2000

Theobald A, Brabänder E: Möglichkeiten der Sekundär- und Primärforschung im Internet. Kaiserslauterer Schriftreihe Marketing, Heft 6/1998

Thinktool! Qualitative knowlegde management : Computerunterstütze Wissensstrukturierung. www.think-tool.de/reg004008.html (zugegriffen am 15/10/2002)

Tinneberg HR: Moderne Fortpflanzungsmedizin - Grundlagen, IVF, ethische und juristische Aspekte; hrsg. v. Tinneberg HR, Ottmar C; Thieme, Stuttgart 1995

Titscher S, Wodak R, Meyer M, Vetter E: Inhaltsanalyse; in: Methoden der Textanalyse, Westdeutscher Verlag, Opladen 1998, 74-91

Tudiver F, Cushman R, Crabtree B, Miller W, Manca D, Brown J: Combining quantitative and qualitative methodologies in primary care. Some examples; in: Primary care research, Volume 1; hrsg. v. Norton PG, Stewart M, Tudiver F, Bass MJ, Dunn EV; Sage, Newbury Park $1991,159-178$

Waegemann CP (2001): Entwicklung und Ausblick. Z Arztl Fortbild Qualitatssich, 95, 601

Weissmann A, Gotlieb L, Ward S (2000): Use of the internet by infertile couples. Fertil Steril, $\underline{73}, 1179-1182$

Werner A (1997): Medien- und Kommunikationsforschung in digitalen Online-Umwelten; in: Computernetze - Ein Medium öffentlicher Kommunikation?; hrsg. v. Beck und Vowe; Wissenschaftsverlag Spiess, Berlin 1997, 227-243

Widman LE, Tong DA (1997): Request for medical advice from patients and families to health care providers who published on the world wide web. Arch Intern Med, 157, 209-212

Wischmann T, Stammer H, Scherg H, Gerhard I, Verres R: Psychosoziale Merkmale von Paaren mit unerfülltem Kinderwunsch aus dem Projekt "Heidelberger KinderwunschSprechstunde"; in: Fruchtbarkeitsstörungen (Jahrbuch der Medizinischen Psychologie, Band 17); hrsg. v. Brähler E, Felder H, Strauß B; Hofgrefe, Göttingen 2000, 245-261

Wright J, Duchesne C, Bissonette F, Benoit J, Girard Y (1991): Psychosocial distress and infertility. Men and women respond differently. Fertil Steril, 55, 100-108 


\section{Danksagung}

Meinem Doktorvater, Priv.-Doz. Dr. disc. Pol. Wolfgang Himmel, danke ich für die Überlassung des Themas der Doktorarbeit, für wichtige Anregungen und hervorragende Betreuung der Arbeit.

Ich danke Herrn Prof. Dr. sc. agr. Hans Wilhelm Michelmann und Herrn Prof. Dr. med. Michael M. Kochen, MPH, FRCGP für Ideen und die kritische Diskussion.

Des Weiteren möchte ich Herrn Ulrich Schneider, dem Betreiber der Website „www.rund-ums-baby.de“, danken, dass er uns gestattete, den Fragebogen auf die Seite des Expertenforums zu platzieren.

Dem Webmaster der Seite, Herrn Christian Schulz, gilt mein Dank für die gute Zusammenarbeit bei der technischen Umsetzung der Fragebogenuntersuchung und dem reibungslosen Datentransfer. Vielen Dank auch an Irene Kühne, Webmaster der Abteilung Allgemeinmedizin, für ihre Unterstützung. 


\section{Lebenslauf}

Am 20.08.1978 wurde ich in Quedlinburg als erstes von zwei Kindern des Diplomlehrers Karl-Heinz Meyer und der Krankenschwester Anne-Heide Meyer, geb. Kniffka, geboren. Von 1985 bis 1991 besuchte ich die Oberschule in Thale und Ballenstedt und anschließend das Gymnasium in Ballenstedt. 1997 legte ich am WolterstorffGymnasium in Ballenstedt das Abitur ab.

Seit dem Wintersemester 1997/98 studiere ich Medizin an der Universität Göttingen. Nach bestandenem zweiten Abschnitt der Ärztlichen Prüfung im April 2003 absolviere ich zurzeit mein Praktisches Jahr im Evangelischen Krankenhaus Göttingen Weende. Im Mai 2004 werde ich voraussichtlich mein Studium mit der dritten Ärztlichen Prüfung abschließen. 